\title{
Borel sets with large squares
}

\author{
by
}

Saharon Shelah (Jerusalem and New Brunswick, N.J.)

\begin{abstract}
For a cardinal $\mu$ we give a sufficient condition $\oplus_{\mu}$ (involving ranks measuring existence of independent sets) for:

$\otimes_{\mu} \quad$ if a Borel set $B \subseteq \mathbb{R} \times \mathbb{R}$ contains a $\mu$-square (i.e. a set of the form $A \times A$ with $|A|=\mu$ ) then it contains a $2^{\aleph_{0}}$-square and even a perfect square,

and also for

$\otimes_{\mu}^{\prime} \quad$ if $\psi \in L_{\omega_{1}, \omega}$ has a model of cardinality $\mu$ then it has a model of cardinality continuum generated in a "nice", "absolute" way.

Assuming MA $+2^{\aleph_{0}}>\mu$ for transparency, those three conditions $\left(\oplus_{\mu}, \otimes_{\mu}\right.$ and $\left.\otimes_{\mu}^{\prime}\right)$ are equivalent, and from this we deduce that e.g. $\bigwedge_{\alpha<\omega_{1}}\left[2^{\aleph_{0}} \geq \aleph_{\alpha} \Rightarrow \neg \otimes_{\aleph_{\alpha}}\right]$, and also that $\min \left\{\mu: \otimes_{\mu}\right\}$, if $<2^{\aleph_{0}}$, has cofinality $\aleph_{1}$.

We also deal with Borel rectangles and related model-theoretic problems.
\end{abstract}

\section{Annotated content}

0. Introduction. We explain results and history and include a list of notation.

1. The rank and the Borel sets. We define some version of the rank for a model, and then $\lambda_{\alpha}(\kappa)$ is the first $\lambda$ such that there is no model with universe $\lambda$, vocabulary of cardinality $\leq \kappa$ and rank $<\alpha$. Now we prove that forcing does not change some ranks of the model, can only decrease others, and c.c.c. forcing changes little. If a Borel or analytic set contains a $\lambda_{\omega_{1}}\left(\aleph_{0}\right)$-square then it contains a perfect square (see 1.12); clearly this gives something only if the continuum is large, that is, at least $\lambda_{\omega_{1}}\left(\aleph_{0}\right)$. On the other hand (see 1.13), if $\mu=\mu^{\aleph_{0}}<\lambda_{\omega_{1}}\left(\aleph_{0}\right)$ then in some c.c.c. forcing extension of $V$ : the continuum is arbitrarily large, and some Borel set contains a $\mu$-square but no $\mu^{+}$-square. Lastly (in 1.15), assuming MA holds we prove exact results (e.g. equivalence of conditions).

2. Some model-theoretic problems. When we restrict ourselves to models of cardinality up to the continuum, $\lambda_{\omega_{1}}\left(\aleph_{0}\right)$ is the Hanf number of $L_{\omega_{1}, \omega}$ (see 2.1).

1991 Mathematics Subject Classification: Primary 03E05, 03E15; Secondary 03E35, $03 \mathrm{C} 55$.

The research was partially supported by "Israeli Science Foundation", founded by the Israeli Academy of Sciences and Humanities. Publication 522. 
Also (see 2.4) if $\psi \in L_{\omega_{1}, \omega}$ has a model realizing many types (say in the countable set $\Delta$ of formulas; many means $\geq \lambda_{\omega_{1}}\left(\aleph_{0}\right)$ ) even after c.c.c. forcing, then

$$
\{\{p: p \text { a complete } \Delta \text {-type realized in } M\}: M \models \psi\}
$$

has two to the continuum members.

We then (2.5) assume $\psi \in L_{\omega_{1}, \omega}$ has a two-cardinal model, say for $(\mu, \kappa)$, and we want to find a $\left(\mu^{\prime}, \aleph_{0}\right)$-model; we need $\lambda_{\omega_{1}}(\kappa) \leq \mu$. Next, more generally, we deal with $\bar{\lambda}$-cardinal models (i.e. we demand that $P_{\zeta}^{M}$ have cardinality $\lambda_{\zeta}$ ). We define ranks (2.8), from them we can formulate sufficient conditions for a transfer theorem and compactness. We can prove that the relevant ranks are (essentially) preserved under c.c.c. forcing as in $\S 1$, and the sufficient conditions hold for $\aleph_{\omega_{1}}$ under GCH.

3. Finer analysis of square existence. In $3.1,3.2$ we define for a sequence $\bar{T}=$ $\left\langle T_{n}: n<\omega\right\rangle$ of trees (i.e. closed sets of the plane) a rank, degsq, whose value is a bound for the size of the square it may contain. We then (3.3) deal with analytic, or more generally $\kappa$-Suslin relations, and use parallel degrees. We then prove that statements on the degrees are related to the existence of squares in $\kappa$-Suslin relations in a way parallel to what we have on Borel relations, using $\lambda_{\alpha}(\kappa)$. We then (3.7-3.11) connect it to the existence of identities for 2-place colourings. In particular, we get results of the form "there is a Borel set $B$ which contains a $\mu$-square iff $\mu<\lambda_{\alpha}\left(\aleph_{0}\right)$ " when MA $+\lambda_{\alpha}\left(\aleph_{0}\right)<2^{\aleph_{0}}$.

4. Rectangles. We deal with the problem of the existence of rectangles in Borel and $\kappa$-Suslin relations. The equivalence of the rank (for models), the existence of perfect rectangles and the model-theoretic statements are more delicate here.

0. Introduction. We first review the old results (from $\S \S 1,2)$. The main one is

$(*)_{1} \quad$ it is consistent that for every successor ordinal $\alpha<\omega_{1}$, there is a Borel subset of $\omega_{2} \times{ }^{\omega} 2$ containing an $\aleph_{\alpha}$-square but no perfect square.

In fact,

$(*)_{1}^{+} \quad$ the result above follows from MA $+2^{\aleph_{0}}>\aleph_{\omega_{1}}$.

For this we define (Definition 1.1) for any ordinal $\alpha$ a property $\operatorname{Pr}_{\alpha}(\lambda ; \kappa)$ of the cardinals $\lambda, \kappa$. The maximal cardinal with the property of $\aleph_{\omega_{1}}$ (i.e. for every small cardinal, c.c.c. forcing adds an example as in $\left.(*)_{1}\right)$ is characterized (as $\lambda_{\omega_{1}}\left(\aleph_{0}\right)$ where $\lambda_{\alpha}(\kappa)=\min \left\{\lambda: \operatorname{Pr}_{\alpha}(\lambda ; \kappa)\right\}$ ); essentially it is not changed by c.c.c. forcing; so in $(*)_{1}$ :

$(*)_{1}^{\prime} \quad$ if in addition $\mathbf{V}=\mathbf{V}_{0}^{\mathbf{P}}$ where $\mathbf{P}$ is a c.c.c. forcing then $\lambda_{\omega_{1}}\left(\aleph_{0}\right) \leq$ $\left(\beth_{\omega_{1}}\right)^{V_{0}}$.

We will generally investigate $\operatorname{Pr}_{\alpha}(\lambda ; \kappa)$, giving equivalent formulations (1.11.6), seeing how fast $\lambda_{\alpha}(\kappa)$ increases, e.g. $\kappa^{+\alpha}<\lambda_{\alpha}(\kappa) \leq \beth_{\omega \times \alpha}(\kappa)$ (in 1.7, 1.8). For two variants we show: $\operatorname{Pr}_{\alpha}^{2}\left(\lambda ; \kappa^{+}\right)\left(\alpha \leq \kappa^{+}\right)$is preserved by $\kappa^{+}$-c.c. forcing, $\operatorname{Pr}_{\alpha}^{l}\left(\lambda ; \kappa^{+}\right) \Rightarrow \operatorname{Pr}_{\alpha}\left(\lambda ; \kappa^{+}\right)$and $\neg \operatorname{Pr}_{\alpha}\left(\lambda ; \kappa^{+}\right)$is preserved by any extension of the universe of set theory. Now $\operatorname{Pr}_{\omega_{1}}\left(\lambda ; \aleph_{0}\right)$ implies that there is no Borel set as above (1.12) but if $\operatorname{Pr}_{\omega_{1}}\left(\lambda ; \aleph_{0}\right)$ fails then some c.c.c. 
forcing adds a Borel set as above (1.13). We cannot in $(*)_{1}$ omit some settheoretic assumption even for $\aleph_{2}$ - see 1.15, 1.16 (add many Cohen reals or many random reals to a universe satisfying e.g. $2^{\aleph_{0}}=\aleph_{1}$; then, in the new universe, every Borel set which contains an $\aleph_{2}$-square also contains a perfect square). We can replace Borel by analytic or even $\kappa$-Suslin (using $\operatorname{Pr}_{\kappa^{+}}(\kappa)$ ).

In $\S 2$ we deal with related model-theoretic questions with less satisfactory results. By 2.1, 2.3, giving a kind of answer to a question from [Sh 49],

$(*)_{2} \quad$ essentially $\lambda=\lambda_{\omega_{1}}\left(\aleph_{0}\right)$ is the Hanf number for models of sentences in $L_{\omega_{1}, \omega}$ when we restrict ourselves to models of cardinality $\leq 2^{\aleph_{0}}$. (What is the meaning of "essentially"? If $\lambda_{\omega_{1}}\left(\aleph_{0}\right) \geq 2^{\aleph_{0}}$ this fails, but if $\lambda_{\omega_{1}}\left(\aleph_{0}\right)<2^{\aleph_{0}}$ it holds.)

In 2.4 we generalize it (the parallel of replacing Borel or analytic sets by $\kappa$-Suslin). We conclude $(2.4(2))$ :

$(*)_{3} \quad$ if $\psi \in L_{\omega_{1}, \omega}\left(\tau_{1}\right), \tau_{0} \subseteq \tau_{1}$ are countable vocabularies, $\Delta \subseteq\{\varphi(x): \varphi \in$ $\left.L_{\omega_{1}, \omega}\left(\tau_{0}\right)\right\}$ is countable and $\psi$ has a model which realizes $\geq \lambda_{\omega_{1}}\left(\aleph_{0}\right)$ complete $(\Delta, 1)$-types then $\mid\left\{\left(M\left\lceil\tau_{0}\right) / \cong: M \models \psi,\|M\|=\lambda\right\} \mid \geq\right.$ $\min \left\{2^{\lambda}, \beth_{2}\right\}$ (for any $\lambda$ ), as we have models as in [Sh a, VII, $\S 4$ ] $=$ [Sh c, VII, §4].

If we allow parameters in the formulas of $\Delta$, and $2^{\aleph_{0}}<2^{\aleph_{1}}$, then $(*)_{3}$ holds too. However, even in the case $2^{\lambda}=2^{\aleph_{0}}$ we prove some results in this direction (see [Sh 262] or better [Sh e, VII, §5]). We then turn to threecardinal theorems etc., trying to continue [Sh 49] (where e.g. $\left(\aleph_{\omega}, \aleph_{0}\right) \rightarrow$ $\left(2^{\aleph_{0}}, \aleph_{0}\right)$ was proved $)$.

We knew those results earlier than or in 1980/1, but failed in efforts to prove the consistency of "ZFC $+\lambda_{\omega_{1}}\left(\aleph_{0}\right)>\aleph_{\omega_{1}}$ " (or proving ZFC $\vdash$ " $\lambda_{\omega_{1}}\left(\aleph_{0}\right)=\aleph_{\omega_{1}}$ "). By the mid-seventies we knew how to get consistency of results like those in $\S 2$ (forcing with $\mathbf{P}$, adding many Cohen reals, i.e. in $\mathbf{V}^{\mathbf{P}}$ getting $(*)_{3}$ for $\left.\lambda=\left(\beth_{\omega_{1}}\right)^{\mathbf{V}}\right)$. This (older proof, not the one used) is closely related to Silver's proof of "every $\Pi_{1}^{1}$-relation with uncountably many equivalence classes has $2^{\aleph_{0}}$ ones" (a deeper one is the proof of Harrington of the Lauchli-Halpern theorem; see a generalization of the Lauchli-Halpern theorem, a partition theorem on ${ }^{\kappa>} 2, \kappa$ large in [Sh 288, §4]).

In fact, about 88 I wrote down for W. Hodges proofs of (a) and (b) stated below.

(a) If, for simplicity, $\mathbf{V}$ satisfies GCH, and we add $>\aleph_{\omega_{1}}$ Cohen reals, then the Hanf number of $L_{\omega_{1}, \omega}$ below the continuum is $\aleph_{\omega_{1}}$.

(b) If $\psi \in L_{\omega_{1}, \omega}\left(\tau_{1}\right)$ and some countable $\Delta \subseteq\left\{\varphi(x): \varphi \in L_{\omega_{1}, \omega}\left(\tau_{0}\right)\right\}$ satisfies: in every forcing extension of $\mathbf{V}, \psi$ has a model which realizes $2^{\aleph_{0}}$ (or at least $\min \left\{2^{\aleph_{0}}, \aleph_{\omega_{1}}\right\}$ ) complete $\Delta$-types, then the conclusion of $(*)_{3}$ above holds. 
Hodges had intended to write it up. Later Hrushovski and Velickovic independently proved the statement (a).

As indicated above, the results had seemed disappointing as the main question "is $\lambda_{\omega_{1}}\left(\aleph_{0}\right)=\aleph_{\omega_{1}}$ ?" is not answered. But Hjorth asked me about (essentially) $(*)_{1}$ which was mentioned in $[\mathrm{HrSh} \mathrm{152]} \mathrm{and} \mathrm{urged} \mathrm{me} \mathrm{to} \mathrm{write}$ this down.

In $\S 3$ we define a degree of Borel sets of the form $\bigcup_{n<\omega} \lim \left(T_{n}\right) \subseteq{ }^{\omega} 2 \times{ }^{\omega} 2$ measuring how close they are to having perfect squares, similarly we define degrees for $\kappa$-Suslin relations, and get results similar to earlier ones under MA and nail the connection between the set of cardinalities of models of $\psi \in L_{\omega_{1}, \omega}$ and having squares. In $\S 4$ we deal with the existence of rectangles.

We can replace $\mathbb{R}^{2}$ by $\mathbb{R}^{3}$ without any difficulty.

In a subsequent paper [Sh 532] which we are writing, we intend to continue the present work and [Sh 202, §5] and deal with: consistency of the existence of co- $\kappa$-Suslin (and even $\Pi_{2}^{1}$-) equivalence relations with many equivalence classes, relationship of $\lambda_{\omega_{1}}^{1}, \lambda_{\omega_{2}}^{3}$ etc., and also try to deal with independence (concerning 2.11 and $4.11(1)$ ) and the existence of many disjoint sections.

I thank Andrzej Rosłanowski for great improvement of the presentation and pointing out gaps, and Andres Villaveces for more corrections.

Notation. Set theory: ${ }^{B} A=\{f: f$ is a function from $B$ to $A\}$, the set of reals is ${ }^{\omega} 2 ; \mathcal{S}_{<\kappa}(A)=[A]^{<\kappa}=\{B \subseteq A:|B|<\kappa\}$.

By a Borel set $B$ we mean the set it defines in the current universe. A $\mu$-square (or a square of size $\mu$ ) is a set of the form $A \times A$, where $A \subseteq \omega_{2}$ and $|A|=\mu$. A $\left(\mu_{1}, \mu_{2}\right)$-rectangle (or rectangle of size $\left(\mu_{1}, \mu_{2}\right)$ ) is a set of the form $A_{1} \times A_{2}$, for some $A_{l} \subseteq{ }^{\omega} 2$ with $\left|A_{l}\right|=\mu_{l}$ (for $l=0,1$ ). A perfect square is $\mathcal{P} \times \mathcal{P}$ with $\mathcal{P} \subseteq{ }^{\omega} 2$ perfect. A perfect rectangle is $\mathcal{P}_{1} \times \mathcal{P}_{2}$ with $\mathcal{P}_{l} \subseteq{ }^{\omega} 2$ perfect. Note that a perfect rectangle is a $\left(2^{\aleph_{0}}, 2^{\aleph_{0}}\right)$-rectangle and a perfect square is a $2^{\aleph_{0}}$-square.

$\mathcal{P}, \mathcal{Q}$ denote perfect sets; $\mathbf{P}, \mathbf{Q}$ denote forcing notions; $P, Q, R$ denote predicates.

A $\kappa$-Suslin set is $\left\{\eta \in{ }^{\omega} 2\right.$ : for some $\nu$ we have $\left.(\eta, \nu) \in \lim (T)\right\}$ for some $(2, \kappa)$-tree $T$ (see below). A $\kappa$-Suslin relation (say an $n$-place relation) is defined similarly.

For $\bar{\lambda}=\left\langle\lambda_{\zeta}: \zeta<\zeta(*)\right\rangle$, a $\bar{\lambda}$-tree is

$$
T \subseteq \bigcup_{n} \prod_{\zeta<\zeta(*)}{ }^{n}\left(\lambda_{\zeta}\right), \quad \text { ordered by } \quad \bar{\eta} \triangleleft \bar{\nu} \Leftrightarrow \bigwedge_{\zeta<\zeta(*)} \eta_{\zeta} \triangleleft \nu_{\zeta} .
$$

We usually let $\bar{\eta} \mid l=\left\langle\eta_{\zeta} \mid l: \zeta<\zeta(*)\right\rangle$. 
For a $\bar{\lambda}$-tree $T$ we define

$$
\lim (T)=\left\{\bar{\eta} \in \prod_{\zeta<\zeta(*)}{ }^{\omega}\left(\lambda_{\zeta}\right): n<\omega \Rightarrow \bar{\eta}\lceil n \in T\}\right.
$$

(where $\left\langle\eta_{\zeta}: \zeta<\zeta(*)\right\rangle\left\lceil n=\left\langle\eta_{\zeta}\lceil n: \zeta<\zeta(*)\rangle\right.\right.$ ) and

$$
\begin{aligned}
\lim ^{*}(T)=\left\{\bar{\eta} \in \prod_{\zeta<\zeta(*)} \omega\left(\lambda_{\zeta}\right):\left(\exists \bar{\eta}^{\prime} \in \lim T\right)(\exists k<\omega)\right. & \\
& \left(\bigwedge_{\zeta<\zeta(*)} \eta_{\zeta}\left\lceil[k, \omega)=\eta_{\zeta}^{\prime}\lceil[k, \omega))\right\} .\right.
\end{aligned}
$$

We will use mainly $(2,2)$-trees and $(2,2, \kappa)$-trees.

Let $\eta \sim_{n} \nu$ mean that $\eta, \nu$ are sequences of ordinals, $\lg (\eta)=\lg (\nu)$ and

$$
(\forall k)[n \leq k<\lg (\eta) \Rightarrow \eta(k)=\nu(k)] .
$$

For a tree $T$ as above, $u \subseteq \zeta(*)$ and $n<\omega$ let

$$
\begin{array}{r}
T^{\left(\sim_{n}, u\right)}=\left\{\bar{\eta}:(\exists k)(\exists \bar{\nu} \in \lim (T))\left[\bar{\nu} \in \prod_{\zeta<\zeta(*)}{ }^{\omega}\left(\lambda_{\zeta}\right) \& \bar{\eta} \in \prod_{\zeta<\zeta(*)}{ }^{k}\left(\lambda_{\zeta}\right)\right.\right. \\
\left.\&(\forall \zeta \in u)\left(\eta_{\zeta} \sim_{n} \nu_{\zeta}\lceil k)\right]\right\} .
\end{array}
$$

Let $\operatorname{Fr}_{n}(\lambda, \mu, \kappa)$ mean that if $F_{\alpha}$ are $n$-place functions from $\lambda$ to $\lambda$ (for $\alpha<\kappa)$ then for some $A \in[\lambda]^{\mu}$ we have

$a_{n} \neq F_{\alpha}\left(a_{0}, \ldots, a_{n-1}\right) \quad$ for distinct $a_{0}, \ldots, a_{n} \in A$ and $\alpha<\kappa$.

Model theory. Vocabularies are denoted by $\tau$, so languages are denoted by e.g. $L_{\kappa, \theta}(\tau)$, models are denoted by $M, N$. The universe of $M$ is $|M|$, its cardinality $\|M\|$. The vocabulary of $M$ is $\tau(M)$ and the vocabulary of $\mathbf{T}$ (a theory or sentence) is $\tau(\mathbf{T}) . R^{M}$ is the interpretation of $R$ in $M$ (for $R \in \tau(M))$. For a model $M$ and a set $B \subseteq M$ we have: $a \in \mathrm{cl}_{<\kappa}(B, M)$ iff for some quantifier free $\varphi=\varphi\left(y, x_{1}, \ldots, x_{n}\right)$ and $b_{1}, \ldots, b_{n} \in B$ we have

$$
M \models \varphi\left[a, b_{1}, \ldots, b_{n}\right] \&\left(\exists^{<\kappa} x\right) \varphi\left(x, b_{1}, \ldots, b_{n}\right) .
$$

Let $\operatorname{cl}_{\kappa}(B, M)=\operatorname{cl}_{<\kappa^{+}}(B, M)$ and $\operatorname{cl}(B, M)=\operatorname{cl}_{<2}(B, M)$. (Note that if $M$ has Skolem functions then $\operatorname{cl}_{<\aleph_{0}}(B, M)=\operatorname{cl}_{<2}(B, M)$ for every $B \subseteq|M|$.) If $\kappa$ is an ordinal we mean $|\kappa|$ (needed just for phrasing absoluteness results; that is, if we use a cardinal $\kappa$ in a universe $V$, and then deal with a generic extension $V^{P}$, then maybe in $V^{P}, \kappa$ is no longer a cardinal but we like to still use it as a parameter). Let $\mathbf{T}$ denote a theory, first order if not said otherwise.

\section{The rank and the Borel sets}

Definition 1.1. (1) For $l<6$, cardinals $\lambda \geq \kappa, \theta$, and an ordinal $\alpha$, let $\operatorname{Pr}_{\alpha}^{l}(\lambda ;<\kappa, \theta)$ mean that for every model $M$ with universe $\lambda$ and vocabulary of cardinality $\leq \theta, \operatorname{rk}^{l}(M ;<\kappa) \geq \alpha$ (defined below), and let $\operatorname{NPr}_{\alpha}^{l}(\lambda ;<\kappa, \theta)$ 
be the negation. Instead of " $<\kappa^{+}$" we may write $\kappa$ (similarly below); if $\kappa=$ $\theta^{+}$we may omit it (so e.g. $\operatorname{Pr}_{\alpha}^{l}(\lambda ; \kappa)$ means $\operatorname{Pr}_{\alpha}^{l}\left(\lambda ;<\kappa^{+}, \kappa\right)$ ); if $\theta=\aleph_{0}, \kappa=$ $\aleph_{1}$ we may omit them. Lastly, let $\lambda_{\alpha}^{l}(<\kappa, \theta)=\min \left\{\lambda: \operatorname{Pr}_{\alpha}^{l}(\lambda ;<\kappa, \theta)\right\}$.

(2) For a model $M$,

$$
\operatorname{rk}^{l}(M ;<\kappa)=\sup \left\{\operatorname{rk}^{l}(w, M ;<\kappa)+1: w \subseteq|M| \text { finite nonempty }\right\}
$$

where $\mathrm{rk}^{l}$ is defined below in part (3).

(3) For a model $M$ and $w \in[M]^{*}:=\{u: u \subseteq|M|$ is finite nonempty $\}$, we shall define below the truth value of $\operatorname{rk}^{l}(w, M ;<\kappa) \geq \alpha$ by induction on the ordinal $\alpha$ (note that if $\operatorname{cl}_{<\kappa}(w, M)=\operatorname{cl}_{2}(w, M)$ for every $w \in[M]^{*}$ then for $l=0,1, \kappa$ can be omitted). Then we can observe:

$(*)_{0} \quad \alpha \leq \beta \& \operatorname{rk}^{l}(w, M ;<\kappa) \geq \beta \Rightarrow \operatorname{rk}^{l}(w, M ;<\kappa) \geq \alpha$,

$(*)_{1} \quad \operatorname{rk}^{l}(w, M ;<\kappa) \geq \delta(\delta$ limit $)$ iff $\bigwedge_{\alpha<\delta} \operatorname{rk}^{l}(w, M ;<\kappa) \geq \alpha$,

$(*)_{2} \quad \operatorname{rk}^{l}(w, M ;<\kappa) \geq 0$ iff $w \in[M]^{*}$ and no $a \in w$ is in $\operatorname{cl}_{<\kappa}(w \backslash\{a\}, M)$.

So we can define $\operatorname{rk}^{l}(w, M ;<\kappa)=\alpha$ as the maximal $\alpha$ such that $\operatorname{rk}^{l}(w, M$; $<\kappa) \geq \alpha$, and $\infty$ if this holds for every $\alpha$ (and -1 whenever $\operatorname{rk}^{l}(w, M ;<\kappa)$ $\geq 0$ ).

Now the inductive definition of $\operatorname{rk}^{l}(w, M ;<\kappa) \geq \alpha$ was already done above for $\alpha=0$ (by $(*)_{2}$ ) and $\alpha$ limit (by $(*)_{1}$ ), so for $\alpha=\beta+1$ we let

$(*)_{3} \quad \operatorname{rk}^{l}(w, M ;<\kappa) \geq \beta+1$ iff (letting $n=|w|, w=\left\{a_{0}, \ldots, a_{n-1}\right\}$ ) for every $k<n$ and a quantifier free formula $\varphi\left(x_{0}, \ldots, x_{n-1}\right)$ (in the vocabulary of $M)$ for which $M \models \varphi\left[a_{0}, \ldots, a_{n-1}\right]$ we have:

CASE $1: l=1$. There are $a_{m}^{i} \in M$ for $m<n, i<2$ such that:

(a) $\operatorname{rk}^{l}\left(\left\{a_{m}^{i}: i<2, m<n\right\}, M ;<\kappa\right) \geq \beta$,

(b) $M \models \varphi\left[a_{0}^{i}, \ldots, a_{n-1}^{i}\right]$ (for $i=1,2$ ), so we can assume there is no repetition in $a_{0}^{i}, \ldots, a_{n-1}^{i}$,

(c) $a_{k}^{0} \neq a_{k}^{1}$ but for $m \neq k$ (such that $m<n$ ) we have $a_{m}^{0}=a_{m}^{1}$.

CASE $2: l=0$. As for $l=1$ but in addition

(d) $\bigwedge_{m} a_{m}=a_{m}^{0}$.

CASE $3: l=3$. We give to $\kappa$ an additional role and the definition is like case 1 but $i<\kappa$; i.e. there are $a_{m}^{i} \in M$ for $m<n, i<\kappa$ such that:

(a) for $i<j<\kappa$ we have $\operatorname{rk}^{l}\left(\left\{a_{m}^{i}, a_{m}^{j}: m<n\right\}, M\right.$; $\left.<\kappa\right) \geq \beta$,

(b) $M \models \varphi\left[a_{0}^{i}, \ldots, a_{n-1}^{i}\right]$ (for $i<\kappa$; so we can assume there are no repetitions in $\left.a_{0}^{i}, \ldots, a_{n-1}^{i}\right)$,

(c) for $i<j<\kappa, a_{k}^{i} \neq a_{k}^{j}$ but for $m \neq k$ (such that $m<n$ ) we have $\bigwedge_{i, j<\kappa} a_{m}^{i}=a_{m}^{j}$.

CASE $4: l=2$. Like case 3 but in addition

(d) $a_{m}=a_{m}^{0}$ for $m<n$. 
CASE $5: l=5$. Like case 3 except that we replace clause (a) by

$\left(\right.$ a) ${ }^{-}$for every function $F$ with $\operatorname{Dom}(F)=\kappa$ and $|\operatorname{Rang}(F)|<\kappa$, for some $i<j<\kappa$ we have $F(i)=F(j)$ and

$$
\operatorname{rk}^{l}\left(\left\{a_{m}^{i}, a_{m}^{j}: m<n\right\}, M ;<\kappa\right) \geq \beta .
$$

CASE $6: l=4$. Like case 4 (i.e. $l=2$ ) with $\left(\right.$ a) ${ }^{-}$instead of (a).

We will actually use the above definition for $l=0$ mainly. As the cardinal $\lambda_{\alpha}^{l}\left(<\aleph_{1}, \aleph_{0}\right)=\lambda_{\alpha}^{l}$ (for $\left.l<2\right)$ may increase when the universe of set theory is extended (new models may be added) we will need some upper bounds which are preserved by suitable forcing. The case $l=2$ provides one (and it is good: it does not increase when the universe is extended by a c.c.c. forcing). The case $l=4$ shows how much we can strengthen the definition, to show for which forcing notions lower bounds for the rank for $l=0$ are preserved. Odd cases show that variants of the definition are immaterial.

Claim 1.2. (1) The truth of each of the statements $\operatorname{Pr}_{\alpha}^{l}(\lambda ;<\kappa, \theta), \mathrm{rk}^{l}(M$; $<\kappa) \geq \alpha, \operatorname{rk}^{l}(w, M ;<\kappa) \geq \alpha$ is preserved if we replace $l=0,2,3,2,2,2,3$, 5,4 by $l=1,3,1,0,1,4,5,1,5$ respectively (i.e. $2 \rightarrow 4,3 \rightarrow 5 \rightarrow 1,0 \rightarrow 1$, $2 \rightarrow 3,4 \rightarrow 5,3 \rightarrow 1,2 \rightarrow 0,2 \rightarrow 1$ ) and also if we decrease $\alpha, \kappa, \theta$ or increase $\lambda$ (the last two only when $M$ is not a parameter). So the corresponding inequality on $\lambda_{\alpha}^{l}(<\kappa, \theta)$ holds.

(2) Also $\operatorname{rk}^{l}\left(w_{1}, M ;<\kappa\right) \geq \operatorname{rk}^{l}\left(w_{2}, M ;<\kappa\right)$ for $w_{1} \subseteq w_{2}$ from $[M]^{*}$.

(3) Also if we expand $M$, the ranks (of $w \in[M]^{*}$, of $M$ ) can only decrease.

(4) If $A \subseteq M$ is defined by a quantifier free formula with parameters from a finite subset $w^{*}$ of $M, M^{+}$is $M$ expanded by the relations defined by quantifier free formulas with parameters from $w^{*}, M^{*}=M^{+} \uparrow A$ (for simplicity $M^{*}$ has relations only) then for $w \in[A]^{*}$ such that $w \nsubseteq w^{*}$ we have $\operatorname{rk}^{l}\left(w, M^{*} ;<\kappa\right) \geq \operatorname{rk}^{l}\left(w \cup w^{*}, M ;<\kappa\right)$. Hence if $w^{*}=\emptyset$, then $\operatorname{rk}^{l}\left(M^{*} ;<k\right) \geq \operatorname{rk}^{l}(M ;<\kappa)$

(5) In $1.1(3)(*)_{2}$, if in the definition of $\mathrm{cl}_{<\kappa}$ we allow any first order formula, this means just expanding $M$ by relations for any first order formula $\varphi(\bar{x})$.

(6) For $l$ odd, $\operatorname{rk}^{l}(w, M ;<\kappa) \geq\left(|\tau(M)|+\aleph_{0}\right)^{+}$implies $\operatorname{rk}^{l}(u, M ;<\kappa)$ $=\infty$.

(7) $\lambda_{\alpha}^{l}(<\kappa, \theta)$ increases $(\leq)$ with $\alpha, \theta$ and decreases with $\kappa$.

(8) There is no difference between $l=4$ and $l=5$.

Proof. Check [e.g. for part (8), we can use a function $F$ such that $(\forall \alpha<\kappa)(F(0) \neq F(1+\alpha)] . \mathbf{m}_{1.2}$ 
Claim 1.3. (1) For $l=0$, if $\alpha=\operatorname{rk}^{l}(M ;<\kappa)(<\infty)$ then for some expansion $M^{+}$of $M$ by $\leq \aleph_{0}+|\alpha|$ relations, for every $w \in[M]^{*}$ we have

$$
\mathrm{rk}^{l+1}\left(w, M^{+} ;<\kappa\right) \leq \operatorname{rk}^{l}(w, M ;<\kappa) .
$$

(2) Similarly for $l=2,4$.

(3) If $\mathbf{V}_{0}$ is a transitive class of $\mathbf{V}_{1}$ (both models of $Z F C$ ) and $M \in \mathbf{V}_{0}$ is a model then:

(a) for $l<4$ :
$(\alpha)\left[\operatorname{rk}^{l}(w, M ;<\kappa)\right]^{\mathbf{V}_{0}} \leq\left[\operatorname{rk}^{l}(w, M ;<\kappa)\right]^{\mathbf{V}_{1}}$ for $w \in[M]^{*}$,
( $\beta)\left[\operatorname{rk}^{l}(M ;<\kappa)\right]^{\mathbf{V}_{0}} \leq\left[\operatorname{rk}^{l}(M ;<\kappa)\right]^{\mathbf{V}_{1}}$,
$(\gamma)$ if $l=0,1$ then equality holds in $(\alpha),(\beta)$,
$(\delta)\left[\lambda_{\alpha}^{l}(\kappa)\right]^{\mathbf{V}_{0}} \leq\left[\lambda_{\alpha}^{l}(\kappa)\right]^{\mathbf{V}_{1}}$ if $l=0,1$.

(b) Assume:

(i) for every $f: \kappa \rightarrow$ Ord from $\mathbf{V}_{1}$ there is $A \in[\kappa]^{\kappa}$ such that $f \nmid A \in \mathbf{V}_{0}$, or at least

(ii) every graph $H$ on $\lambda$ from $\mathbf{V}_{0}$ which in $\mathbf{V}_{1}$ has a complete subgraph of size $\kappa$, has such a subgraph in $\mathbf{V}_{0}$, which holds if

(ii) $^{+} \mathbf{V}_{1}=\mathbf{V}_{0}^{\mathbf{P}}$ where $\mathbf{P}$ is a forcing notion satisfying the $\kappa$ Knaster Condition.

Then for $l=2,3$, in $(\alpha),(\beta)$ (of (a)) above equalities hold, and the inequality in $(\delta)$ holds.

(c) Assume $\mathbf{V}_{1}=\mathbf{V}_{0}^{\mathbf{P}}$ where $\mathbf{P}$ is $\kappa$-2-linked. Then for $l=4,5$, in clauses $(\alpha),(\beta)$ (of (a)) above we have equality, and the inequality in $(\delta)$ holds.

Proof. (1) For $\beta<\alpha, n<\omega$, a quantifier free formula $\varphi=\varphi\left(x_{0}, \ldots\right.$ $\left.\ldots, x_{n-1}\right)$ and $k<n$ let

$$
\begin{gathered}
R_{\beta}^{n}=\left\{\left\langle a_{0}, \ldots, a_{n-1}\right\rangle: a_{m} \in M \text { for } m<n\right. \text { and } \\
\left.\beta=\operatorname{rk}^{l}\left(\left\{a_{0}, \ldots, a_{n-1}\right\}, M ;<\kappa\right)\right\}, \\
R_{\beta, \varphi}^{n, k}=\left\{\left\langle a_{0}, \ldots, a_{n-1}\right\rangle \in R_{\beta}^{n} M \models \varphi\left[a_{0}, \ldots, a_{n-1}\right]\right. \text { and } \\
\text { for no } a_{k}^{1} \in|M| \backslash\left\{a_{0}, \ldots, a_{n-1}\right\} \text { do we have } \\
(\alpha) M \models \varphi\left[a_{0}, \ldots, a_{k-1}, a_{k}^{1}, a_{k+1}, \ldots, a_{n-1}\right], \\
\left.(\beta) \operatorname{rk}^{l}\left(\left\{a_{m}: m<n\right\} \cup\left\{a_{k}^{1}\right\}, M ;<\kappa\right) \geq \beta\right\}, \\
M^{+}=\left(M, \ldots, R_{\beta}^{n}, R_{\beta, \varphi}^{n, k}, \ldots\right)_{\beta<\alpha, n<\omega, k<n, \varphi} .
\end{gathered}
$$

Check (or see more details in the proof of 1.10 below).

(2) Similarly. 
(3) The proof should be clear (for (b), look at Definition 1.1, case 3; the graph is $\{(i, j)$ : clause (a) there holds $\})$.

REMARK 1.4. (1) In 1.3(1) we can omit " $\alpha=\operatorname{rk}^{l}(M ;<\kappa)$ " but then we must weaken the conclusion to

$$
\operatorname{rk}^{l+1}\left(w, M^{+} ;<\kappa\right) \leq \operatorname{rk}^{l}(w, M ;<\kappa) \quad \text { or both are }>\alpha .
$$

(2) Similarly in 1.3(2).

Conclusion 1.5. (1) $\operatorname{Pr}_{\omega_{1}}^{0}(\lambda) \Leftrightarrow \operatorname{Pr}_{\omega_{1}}^{1}(\lambda) \Leftarrow \operatorname{Pr}_{\omega_{1}}^{4}(\lambda) \Leftrightarrow \operatorname{Pr}_{\omega_{1}}^{5}(\lambda) \Leftarrow$ $\operatorname{Pr}_{\omega_{1}}^{2}(\lambda) \Leftrightarrow \operatorname{Pr}_{\omega_{1}}^{3}(\lambda)$.

(2) If $\alpha \leq \kappa^{+}$then $\operatorname{Pr}_{\alpha}^{0}(\lambda ; \kappa) \Leftrightarrow \operatorname{Pr}_{\alpha}^{1}(\lambda ; \kappa) \Leftarrow \operatorname{Pr}_{\alpha}^{4}(\lambda ; \kappa) \Leftrightarrow \operatorname{Pr}_{\alpha}^{5}(\lambda ; \kappa) \Leftarrow$ $\operatorname{Pr}_{\alpha}^{2}(\lambda ; \kappa) \Leftrightarrow \operatorname{Pr}_{\alpha}^{3}(\lambda ; \kappa)$.

(3) For $\alpha \leq \kappa^{+}, \lambda_{\alpha}^{l}(\kappa)=\lambda_{\alpha}^{l+1}(\kappa)$ for $l=0,2,4$, and $\lambda_{\alpha}^{0}(\kappa) \leq \lambda_{\alpha}^{4}(\kappa) \leq$ $\lambda_{\alpha}^{2}(\kappa)$.

(4) For $\alpha \geq \kappa^{+}$and $l=0,2$, 4 we have $\lambda_{\alpha}^{l+1}(\kappa)=\lambda_{\kappa^{+}}^{l+1}(\kappa)$.

Proof. (1) By (2).

(2) For $\alpha=\kappa^{+}$this follows from its holding for every $\alpha<\kappa^{+}$. For $\alpha<\kappa^{+}$ and $l=0,2,4$ we know that $\operatorname{NPr}_{\alpha}^{l}(\lambda ; \kappa) \Rightarrow \operatorname{NPr}_{\alpha}^{l+1}(\lambda ; \kappa)$ by 1.3(1),(2), and $\operatorname{Pr}_{\alpha}^{l}(\lambda ; \kappa) \Rightarrow \operatorname{Pr}_{\alpha}^{l+1}(\lambda ; \kappa)$ by $1.2(1)$; together $\operatorname{Pr}_{\alpha}^{l}(\lambda ; \kappa) \Leftrightarrow \operatorname{Pr}_{\alpha}^{l+1}(\lambda ; \kappa)$. Now $\operatorname{Pr}_{\alpha}^{3}(\lambda ; \kappa) \Rightarrow \operatorname{Pr}_{\alpha}^{5}(\lambda ; \kappa) \Rightarrow \operatorname{Pr}_{\alpha}^{1}(\lambda ; \kappa)$ by $1.2(1)$; altogether we finish. (By 2.1 we know more.)

(3) Follows from part (2) and the definition.

(4) By 1.2(6).

Convention 1.6. Writing $\operatorname{Pr}_{\alpha}(\lambda ; \kappa)$ for $\alpha \leq \kappa^{+}$(omitting $l$ ) we mean $l=0$. Similarly $\lambda_{\alpha}(<\kappa, \theta)$ and so $\lambda_{\alpha}(\kappa)$ etc.

Claim 1.7. Let $l \in\{0,2,4\}$.

(1) $\operatorname{NPr}_{\alpha+1}^{l}\left(\kappa^{+\alpha} ; \kappa\right)$.

(2) If $\alpha$ is a limit ordinal $<\kappa^{+}$(in fact, $\aleph_{0} \leq \operatorname{cf}(\alpha)<\kappa^{+}$suffice) and $\operatorname{NPr}_{\beta}\left(\lambda_{\beta} ; \kappa\right)$ for $\beta<\alpha$ then $\operatorname{NPr}_{\alpha+1}^{l}\left(\sum_{\beta<\alpha} \lambda_{\beta} ; \kappa\right)$.

(3) If $\operatorname{NPr}_{\alpha}^{l}(\lambda ; \kappa)$ then $\operatorname{NPr}_{\alpha+1}^{l}\left(\lambda^{+} ; \kappa\right)$.

(4) If $\operatorname{NPr}_{\alpha}^{l}(\mu ; \kappa)$ for every $\mu<\lambda$ then $\operatorname{NPr}_{\alpha+1}^{l}(\lambda ; \kappa)$.

P r o o f. (1) Prove by induction on $\alpha<\kappa^{+}$, for $\alpha=0$ use a model in which every element is definable (e.g. an individual constant) so $\operatorname{rk}(w ; M)=-1$ for $w \in[M]^{*}$ and hence $\operatorname{rk}^{l}(M)=0$ and consequently $\operatorname{NPr}_{1}^{l}(\kappa ; \kappa)$; for $\alpha$ limit use part (2) and for $\alpha$ successor use part (3).

(2) Let $M_{\beta}$ witness $\operatorname{NPr}_{\beta}\left(\lambda_{\beta} ; \kappa\right)$ for $\beta<\alpha$, i.e. $\operatorname{rk}^{l}\left(M_{\beta} ; \kappa\right)<\beta$ and $M_{\beta}$ has universe $\lambda_{\beta}$ and $\left|\tau\left(M_{\beta}\right)\right| \leq \kappa$. Without loss of generality $\left\langle\tau\left(M_{\beta}\right): \beta<\alpha\right\rangle$ are pairwise disjoint and disjoint from $\left\{P_{\beta}: \beta<\alpha\right\}$. Let $M$ have universe $\lambda:=\sum_{\beta<\alpha} \lambda_{\beta}, P_{\beta}^{M}=\lambda_{\beta}$, and $M\left\lceil\lambda_{\beta}\right.$ expand $M_{\beta}$ and $|\tau(M)| \leq$ $|\alpha|+\sum_{\beta \leq \alpha}\left|\tau\left(M_{\beta}\right)\right| \leq \kappa$. By 1.2(3),(4), for $w \in\left[\lambda_{\beta}\right]^{*}, \operatorname{rk}^{l}(w, M ; \kappa) \leq$ 
$\operatorname{rk}^{l}\left(w, M_{\beta} ; \kappa\right)<\beta \leq \alpha$. But $w \in[|M|]^{*}$ implies $\bigvee_{\beta<\alpha} w \in\left[\lambda_{\beta}\right]^{*}$. Clearly $\operatorname{rk}(M ; \kappa) \leq \alpha$ and hence $\operatorname{NPr}_{\alpha+1}^{l}(\lambda ; \kappa)$.

(3) We define $M^{+}$such that each $\gamma \in\left[\lambda, \lambda^{+}\right)$codes on $\{\zeta: \zeta<\gamma\}$ an example for $\operatorname{NPr}_{\alpha}(|\gamma| ; \kappa)$. More elaborately, let $M$ be a model with universe $\lambda$ such that $\operatorname{rk}^{l}(M ; \kappa)<\alpha$. Let $\tau(M)$ be $\left\{R_{i}: i<i^{*} \leq \kappa\right\}$, $R_{i}$ an $n(i)$-place predicate (as we replace function symbols and individual constants by predicates), $R_{0}$ is a 0 -nary predicate representing "the truth". For $\gamma \in\left[\lambda, \lambda^{+}\right)$let $f_{\gamma}$ be a one-to-one function from $\gamma$ onto $\lambda$. Define $\tau^{+}=\left\{R_{i}, Q_{i}: i<i^{*} \leq \kappa\right\}$ where $R_{i}$ is $n(i)$-place and $Q_{i}$ is $(n(i)+1)$-place. So $\left|\tau^{+}\right| \leq \kappa$. We define a $\tau^{+}$-model $M^{+}$: the universe is $\lambda^{+}$, $R_{i}^{M^{+}}=R_{i}^{M}, Q_{i}^{M^{+}}=\left\{\left\langle\alpha_{0}, \ldots, \alpha_{n(i)}\right\rangle: \alpha_{n(i)} \in\left[\lambda, \lambda^{+}\right), \bigwedge_{l<n(i)} \alpha_{l}<\alpha_{n(i)}\right.$ and $\left.\left\langle f_{\alpha_{n(i)}}\left(\alpha_{0}\right), \ldots, f_{\alpha_{n(i)}}\left(\alpha_{n(i)-1}\right)\right\rangle \in R_{i}^{M}\right\}$ (so $\left.Q_{0}^{M^{+}}=\left[\lambda, \lambda^{+}\right)\right)$. Now note that:

(a) for $w \in[\lambda]^{*}, \operatorname{rk}^{l}\left(w, M^{+} ; \kappa\right) \leq \operatorname{rk}^{l}(w, M ; \kappa)$,

(b) if $\emptyset \neq w \subseteq \gamma \in\left[\lambda, \lambda^{+}\right)$then $\operatorname{rk}^{l}\left(w \cup\{\gamma\}, M^{+} ; \kappa\right) \leq \operatorname{rk}^{l}\left(f_{\gamma}^{\prime \prime}[w], M ; \kappa\right)$

(easy to check). So if $\gamma<\lambda^{+}$then

$(*)_{1} \quad \gamma<\lambda \Rightarrow \operatorname{rk}^{l}\left(\{\gamma\}, M^{+} ; \kappa\right) \leq \operatorname{rk}^{l}(\{\gamma\}, M ; \kappa)<\operatorname{rk}^{l}(M ; \kappa)$,

$(*)_{2} \quad \gamma \in\left[\lambda, \lambda^{+}\right) \& \beta \geq \operatorname{rk}^{l}(M ; \kappa) \Rightarrow \operatorname{rk}^{l}\left(\{\gamma\}, M^{+} ; \kappa\right) \leq \beta$.

(Why $(*)_{2}$ ? Assume not and let $\kappa^{0}=2, \kappa^{2}=\kappa^{4}=\kappa^{+}$. If $\left\langle\gamma_{i}: i<\kappa^{l}\right\rangle$ strictly increasing witnesses $\operatorname{rk}^{l}\left(\{\gamma\}, M^{+}\right) \geq \beta+1$ for the formula $Q_{0}(x)$ then for some $i<j<\kappa^{l}$ we have $\operatorname{rk}^{l}\left(\left\{\gamma_{i}, \gamma_{j}\right\}, M^{+}\right) \geq \beta$ and applying (b) with $\left\{\gamma_{i}\right\}, \gamma_{j}$ here standing for $w, \gamma$ there we get $\operatorname{rk}^{l}\left(\left\{f_{\gamma_{j}}\left(\gamma_{i}\right)\right\}, M\right) \geq \beta$, hence $\beta+1 \leq \operatorname{rk}^{l}(M)$, a contradiction.)

Hence

$(*)_{3} \quad \operatorname{rk}^{l}\left(M^{+} ; \kappa\right) \leq \operatorname{rk}^{l}(M ; \kappa)+1$.

As $\operatorname{rk}^{l}\left(M^{+} ; \kappa\right)<\alpha$, clearly $M^{+}$witnesses $\operatorname{NPr}_{\alpha+1}\left(\lambda^{+} ; \kappa\right)$.

(4) Like (3).

Conclusion 1.8. Remembering $\lambda_{\alpha}(\kappa)=\min \left\{\lambda: \operatorname{Pr}_{\alpha}(\lambda ; \kappa)\right\}$ we have:

(1) for $\alpha$ a limit ordinal, $\lambda_{\alpha}(\kappa) \leq \beth_{\alpha}(\kappa)$ and even $\lambda_{\alpha}^{2}(\kappa) \leq \beth_{\alpha}(\kappa)$,

(2) for $l$ even, $\left\langle\lambda_{\alpha}^{l}(\kappa): 0<\alpha<\infty\right\rangle$ is strictly increasing, and for a limit ordinal $\delta, \lambda_{\delta}(\kappa)=\sup _{\alpha<\delta} \lambda_{\alpha}(\kappa)$,

(3) $\lambda_{0}(\kappa)=\lambda_{1}(\kappa)=\kappa, \lambda_{2}(\kappa)=\kappa^{+}, \kappa^{+n} \leq \lambda_{n}(\kappa)<\kappa^{+\omega}$ and $\lambda_{\omega}(\kappa)=$

REMARK 1.9. $\lambda_{\omega \times \alpha}^{2}(\kappa) \leq \beth_{\omega \times \alpha}(\kappa)$ is proved below essentially like the Morley omitting types theorem (see $[\mathrm{Mo}],[\mathrm{CK}]$ or $[\mathrm{Sh}$ a, VII, $\S 5]=[\mathrm{Sh}$ c, VII, §5]). 
Pr o of (of Conclusion 1.8). (1) We prove by induction on $\alpha$ that for every ordinal $\beta<\alpha$, model $M$ with $|\tau(M)| \leq \kappa$, and $A \subseteq|M|$ with $|A| \geq \beth_{\omega \times \alpha}(\kappa)$, and $m, n<\omega$, there is $w \subseteq A$ with $|w|=n$ such that $\operatorname{rk}^{2}(w, M ; \kappa) \geq$ $\omega \times \beta+m$.

For $\alpha=0$ and $\alpha$ limit this is immediate. For $\alpha=\gamma+1$ (and $M, A, \beta, n$, $m$ as above), applying the Erdös-Rado theorem we can find distinct $a_{i} \in A$ for $i<\beth_{\omega \times \gamma}(\kappa)^{++}$such that:

(a) for all $i_{0}<\ldots<i_{m+n}$ the quantifier free type $\left\langle a_{i_{0}}, \ldots, a_{i_{n+m}}\right\rangle$ in $M$ is the same,

(b) for each $k \leq m+n$ and every $i_{0}<\ldots<i_{n+m-k}<\beth_{\omega \times \gamma}(\kappa)$, the ordinal $\min \left\{\omega \times \alpha, \operatorname{rk}^{2}\left(\left\{a_{i_{0}}, \ldots, a_{i_{n+m-k}}\right\}, M ; \kappa\right)\right\}$ is the same.

By the induction hypothesis, in clause (b) the value is $\geq \omega \times \gamma$. Hence we can prove, by induction on $k \leq m+n$, that $\operatorname{rk}^{2}\left(\left\{a_{i_{0}}, \ldots, a_{i_{n+m-k}}\right\}, M ; \kappa\right) \geq$ $\omega \times \gamma+k$ whenever $i_{0}<\ldots<i_{m+n-k}<\beth_{\omega \times \gamma}(\kappa)$. For $k=0$ this holds by the previous sentence, for $k+1$ use the definition and the induction hypothesis, for $\mathrm{rk}^{2}$ note that by clause (b) without loss of generality $i_{l}+\kappa^{+}<i_{l+1}$ and $a_{i_{l}+\zeta}$ for $\zeta<\kappa^{+}$are well defined. For $k=m$ we are done.

(2) The sequence is increasing by 1.2(1), strict by 1.7(4), continuous because, for limit $\delta$, as on the one hand $\lambda_{\delta}^{l}(\kappa) \geq \sup _{\alpha<\delta} \lambda_{\alpha}^{l}(\kappa)$ since $\lambda_{\delta}^{l}(\kappa) \geq$ $\lambda_{\alpha}^{l}(\kappa)$ for $\alpha<\delta$, and on the other hand if $M$ is a model with universe $\lambda:=$ $\sup _{\alpha<\delta} \lambda_{\alpha}(\kappa)$ and $|\tau(M)| \leq \kappa$ then $\alpha<\delta \Rightarrow \operatorname{rk}^{l}(M ; \kappa) \geq \operatorname{rk}^{l}\left(M \uparrow \lambda_{\alpha} ; \kappa\right) \geq \alpha$ hence $\operatorname{rk}^{l}(M ; \kappa) \geq \delta$. So $\operatorname{Pr}_{\alpha}(\lambda ; \kappa)$ hence $\lambda \geq \lambda_{\delta}^{l}(\kappa)$ so $\sup _{\alpha<\delta} \lambda_{\alpha}^{l}(\kappa)=\lambda \geq$ $\lambda_{\delta}^{l}$; altogether, we are done.

(3) By [Sh 49] (for the last two clauses; the first two are trivial), will not be really used here.

Claim 1.10. (1) Assume $\mathbf{P}$ is a forcing notion satisfying the $\kappa^{+}$-c.c. If $\operatorname{Pr}_{\alpha}^{3}(\lambda ; \kappa)$ and $\alpha \leq \kappa^{+}$, then this also holds in $\mathbf{V}^{\mathbf{P}}$.

(2) If $\mathbf{P}$ is a $\kappa^{+}-2$-linked forcing notion (or just: if $p_{i} \in \mathbf{P}$ for $i<\kappa^{+}$ then for some $F: \kappa^{+} \rightarrow \kappa, F(i)=F(j) \Rightarrow p_{i}, p_{j}$ compatible), and $\alpha \leq \kappa^{+}$ and $\operatorname{Pr}_{\alpha}^{5}(\lambda ; \kappa)$, then this holds in $\mathbf{V}^{\mathbf{P}}$.

REMARK 1.11. (1) $\operatorname{NPr}_{\alpha}(\lambda ; \kappa)$ is of course preserved by any extension as the $\operatorname{ranks} \operatorname{rk}^{l}(M ; \kappa), \operatorname{rk}^{l}(w, M ; \kappa)$ are absolute for $l=0,1$ (see 1.3(3)). But the forcing can add new models.

(2) So for $\alpha \leq \kappa^{+}$we have $\lambda_{\alpha}(\kappa) \leq \lambda_{\alpha}^{4}(\kappa) \leq \lambda_{\alpha}^{2}(\kappa)$, and a $\kappa^{+}$-c.c. forcing notion can only increase the first (by $1.3(3)(\mathrm{a})(\delta)$ ) and decrease the third by $1.10(1)$; a $\kappa^{+}-2$-linked one fixes the second and third (as it can only decrease it by $1.10(1)$ and can only increase it by $1.3(3)(\mathrm{c})$.

(3) We can deal similarly with $\operatorname{Pr}_{\alpha}^{l}(\lambda ;<\kappa, \theta)$, here and in $1.3-1.8$.

Pr o of (of Claim 1.10). We can concentrate on (1); anyhow let $l=\{3,5\}$ (for part (1) we use $l=3$, for part (2) we shall use $l=5$, we shall return to 
it later). Assume $\operatorname{Pr}_{\alpha}^{3}(\lambda ; \kappa)$ fails in $\mathbf{V}^{\mathbf{P}}$. So for some $p^{*} \in \mathbf{P}$ and $\alpha_{0}<\alpha$ we have

$p^{*} \vdash_{\mathbf{P}}$ " $M$ is a model with universe $\lambda$, vocabulary $\mathcal{\sim}$ of cardinality $\leq \kappa$ and $\operatorname{rk}^{l}(\underset{\sim}{M} ; \kappa)=\alpha_{0} "$.

Without loss of generality, every quantifier free formula $\varphi\left(x_{0}, \ldots, x_{n-1}\right)$ is equivalent to one of the form $R\left(x_{0}, \ldots, x_{n-1}\right)$, and $\underset{\sim}{\tau}=\left\{R_{n, \zeta}: n<\omega\right.$, $\zeta<\kappa\}$ with $R_{n, \zeta}$ an $n$-place predicate. Note that necessarily $\alpha_{0}<\kappa^{+}$, hence $\left|\alpha_{0}\right| \leq \kappa$.

As we can replace $\mathbf{P}$ by $\mathbf{P}\left\lceil\left\{q \in \mathbf{P}: p^{*} \leq q\right\}\right.$, we can assume $p^{*}$ is the minimal member of $\mathbf{P}$. Now for nonzero $n<\omega, k<n, \zeta<\kappa$ and $\beta<\alpha_{0}$ (or $\beta=-1$ ) we define an $n$-place relation $R_{n, \zeta, \beta, k}$ on $\lambda$ by $R_{n, \zeta, \beta, k}=\left\{\left\langle a_{0}, \ldots, a_{n-1}\right\rangle: a_{m} \in \lambda\right.$ with no repetitions and for some $p \in \mathbf{P}$, $p \Vdash_{\mathbf{P}}$ “ $M \models R_{n, \zeta}\left[a_{0}, \ldots, a_{n-1}\right] \& \operatorname{rk}^{l}\left(\left\{a_{0}, \ldots, a_{n-1}\right\}, M ; \kappa\right)=\beta$, where "not $\operatorname{rk}^{3}\left(\left\{a_{0}, \ldots, a_{n-1}\right\}, M ; \kappa\right) \geq \beta+1$ " is witnessed by $\varphi=R_{n, \zeta}$ and $\left.k "\right\}$.

Let $M^{+}=\left(\lambda, \ldots, R_{n, \zeta, \beta, k}, \ldots\right)_{n<\omega, \zeta<\kappa, \beta<\alpha_{0}, k<n}$, so $M^{+}$is a model in $\mathbf{V}$ with universe $\lambda$ and vocabulary of cardinality $\leq \kappa$. It suffices to prove that for $\beta<\alpha_{0}$,

$\otimes_{\beta} \quad$ if $w=\left\{a_{0}, \ldots, a_{n-1}\right\} \in\left[M^{+}\right]^{*}, M^{+} \models R_{n, \zeta, \beta, k}\left[a_{0}, \ldots, a_{n-1}\right]$

then $\operatorname{rk}^{l}\left(\left\{a_{0}, \ldots, a_{n-1}\right\}, M^{+} ; \kappa\right) \leq \beta$

(note that by the choice of $\underset{\sim}{M}$ and $R_{n, \zeta, \beta, k}$, if $w \in\left[M^{+}\right]^{*}$ then for some $n, \zeta, \beta, k$ we have $\left.M^{+} \models R_{n, \zeta, \beta, k}\left[a_{0}, \ldots, a_{n-1}\right]\right)$.

This we prove by induction on $\beta$, so assume the conclusion fails, that is,

$$
\operatorname{rk}^{l}\left(\left\{a_{0}, \ldots, a_{n-1}\right\}, M^{+} ; \kappa\right) \geq \beta+1
$$

(and eventually we shall get a contradiction). By the definition of $\mathrm{rk}^{3}$ applied to $\varphi=R_{n, \zeta, \beta, k}, \beta$ and $k$ we know that there are $a_{m}^{i}$ (for $m<n, i<\kappa^{+}$) as in Definition 1.1(3), case $l=3$. In particular, $M^{+} \models R_{n, \zeta, \beta, k}\left[a_{0}^{i}, \ldots, a_{n-1}^{i}\right]$. So for each $i<\kappa^{+}$by the definition of $R_{n, \zeta, \beta, k}$ necessarily there is $p_{i} \in \mathbf{P}$ such that

$$
\begin{aligned}
p_{i} \Vdash_{\mathbf{P}} & \text { “ } \underset{\sim}{M} \models R_{n, \zeta}\left[a_{0}^{i}, \ldots, a_{n-1}^{i}\right], \operatorname{rk}^{l}\left(\left\{a_{0}^{i}, \ldots, a_{n-1}^{i}\right\}, \underset{\sim}{M} ; \kappa\right)=\beta \\
& \text { and } \operatorname{rk}^{l}\left(\left\{a_{0}^{i}, \ldots, a_{n-1}^{i}\right\}, \underset{\sim}{M} ; \kappa\right) \geq \beta+1 \text { is witnessed by } \\
& \varphi=R_{n, \zeta} \text { and } k " .
\end{aligned}
$$

For part (1), as $\mathbf{P}$ satisfies the $\kappa^{+}$-c.c., for some $q \in \mathbf{P}, q \Vdash \Perp_{\sim}^{Y}=\{i$ : $\left.p_{i} \in G_{\mathbf{P}}\right\}$ has cardinality $\kappa^{+}$" (in fact, $p_{i}$ forces it for every large enough $i$ ). Looking at the definition of the rank in $\mathbf{V}^{\mathbf{P}}$ we see that $\left\langle\left\langle a_{0}^{i}, \ldots, a_{n-1}^{i}\right\rangle: i \in\right.$ $\underset{\sim}{Y}\rangle$ cannot be a witness for "the demand for $\operatorname{rk}^{3}\left(\left\{a_{0}^{i_{0}}, \ldots, a_{n-1}^{i_{0}}\right\}, M \sim \kappa\right)>\beta$ 
for $R_{n, \zeta, k}$ holds" for any (or some) $i_{0} \in \underset{\sim}{Y}$, so for part (1),

(*) $\quad q \Vdash_{\mathbf{P}}$ " $\mathrm{rk}^{3}\left(\left\{a_{0}^{i}, \ldots, a_{n-1}^{i}, a_{k}^{j}\right\}, \underset{\sim}{M} ; \kappa\right)<\beta$ for some $i \neq j$ in $\underset{\sim}{Y}$ "

(as the demand on equalities holds trivially).

As we can increase $q$, we can assume that $q$ forces a value to those $i, j$, hence without loss of generality for some $n(*)=n+1<\omega, \zeta(*)<\kappa$ and $\beta(*)<\beta$ and for $k(*)<n+1$ we have

$$
\begin{aligned}
q \Vdash_{\mathbf{P}} & \text { " } \mathrm{rk}^{3}\left(\left\{a_{0}^{i}, \ldots, a_{n-1}^{i}, a_{k}^{j}\right\}, \underset{\sim}{M} ; \kappa\right)=\beta(*), \text { and } \\
& \operatorname{rk}^{3}\left(\left\{a_{0}^{i}, \ldots, a_{n-1}^{i}, a_{k}^{j}\right\}, M ; \kappa\right) \nsupseteq \beta(*)+1 \text { is witnessed by } \\
& \varphi=R_{n(*), \zeta(*)}\left(x_{0}, \ldots, x_{n}\right) \text { and } k(*) " .
\end{aligned}
$$

Hence by the definition of $R_{n(*), \zeta(*), \beta(*), k(*)}$ we have

$$
M^{+} \models R_{n(*), \zeta(*), \beta(*), k(*)}\left[a_{0}^{i}, \ldots, a_{n-1}^{i}, a_{k}^{j}\right] .
$$

As $\beta(*)<\beta$, by the induction hypothesis $\otimes_{\beta(*)}$ holds and hence

$$
\operatorname{rk}^{3}\left(\left\{a_{0}^{i}, \ldots, a_{n-1}^{i}, a_{k}^{j}\right\}, M^{+} ; \kappa\right) \leq \beta(*) ;
$$

but this contradicts the choice of $a_{m}^{i}\left(m<n, i<\kappa^{+}\right)$above (i.e. clause (a) of Definition 1.1(3), case $l=3$ ). This contradiction finishes the induction step in the proof of $\otimes_{\beta}$, hence the proof of 1.10(1).

For part (2), we have $\left\langle p_{i}: i<\kappa^{+}\right\rangle$as above. In $\mathbf{V}^{\mathbf{P}}$, if $\underset{\sim}{Y}=\{i$ : $\left.p_{i} \in G_{\mathbf{P}}\right\}$ has cardinality $\kappa^{+}$, then $\left\langle\left\langle a_{0}^{i}, \ldots, a_{n-1}^{i}\right\rangle: i \in \underset{\sim}{Y}\right\rangle$ cannot witness $\operatorname{rk}^{5}\left(\left\{a_{0}, \ldots, a_{n-1}\right\}, M ; \kappa\right) \geq \beta+1$ so there is a function $\underset{\sim}{F^{0}}: \underset{\sim}{Y} \rightarrow \kappa$ witnessing it; i.e.

$$
\begin{aligned}
& \Vdash_{\mathbf{P}} \text { "if }|\underset{\sim}{Y}|=\kappa^{+} \text {then } \\
& \qquad \begin{array}{l}
i \in \underset{\sim}{Y} \& j \in \underset{\sim}{Y} \& i \neq j \& \underset{\sim}{F^{0}}(i)={\underset{\sim}{F}}^{0}(j) \Rightarrow \\
\quad \beta>\operatorname{rk}^{5}\left(\left\{a_{0}^{i}, \ldots, a_{n-1}^{i}\right\} \cup\left\{a_{0}^{j}, \ldots, a_{n-1}^{j}\right\}, M ; \kappa\right) " .
\end{array}
\end{aligned}
$$

If $|\underset{\sim}{Y}| \leq \kappa$, let $\underset{\sim}{F^{0}}: \underset{\sim}{Y} \rightarrow \kappa$ be one-to-one. Let $p_{i} \leq q_{i} \in \mathbf{P}, q_{i} \Vdash{\underset{\sim}{F}}^{0}(i)=\gamma_{i}$. As $\mathbf{P}$ is $\kappa^{+}$-2-linked, for some function $F^{1}: \kappa^{+} \rightarrow \kappa$ we have $\left(\forall i, j<\kappa^{+}\right)$ $\left(F^{1}(i)=F^{1}(j) \Rightarrow q_{i}, q_{j}\right.$ are compatible in $\left.\mathbf{P}\right)$. We now define a function $F$ from $\underset{\sim}{Y}$ to $\kappa$ by $F(i)=\operatorname{pr}\left(\gamma_{i}, F^{1}(i)\right.$ ) (you can use any pairing function pr on $\kappa)$. So if $i<j<\kappa^{+}$and $F(i)=F(j)$ then there is $q_{i, j}$ such that $\mathbf{P} \models$ " $q_{i} \leq q_{i, j} \& q_{j} \leq q_{i, j}$ ", hence $q_{i, j} \Vdash_{\mathbf{P}}$ " $\mathrm{rk}^{5}\left(\left\{a_{0}^{i}, \ldots, a_{n-1}^{i}, a_{k}^{j}\right\}, \underset{\sim}{M} ; \kappa\right)<\beta$ ", so possibly increasing $q_{i, j}$, for some $\beta_{i, j}<\beta, \zeta_{i, j}<\kappa$, and $k_{i, j}<n$ we have $q_{i, j} \Vdash " \mathrm{rk}^{5}\left(\left\{a_{0}^{i}, \ldots, a_{n-1}^{i}, a_{k}^{j}\right\}, \underset{\sim}{M} ; \kappa\right)=\beta_{i, j}$, and $\operatorname{rk}^{5}\left(\left\{a_{0}^{i}, \ldots, a_{n-1}^{i}, a_{k}^{j}\right\}\right) \nsupseteq$ $\beta_{i, j}+1$ is witnessed by $\varphi=R_{n+1, \zeta_{i, 1}}\left(x_{0}, \ldots, x_{n}\right)$ and $k_{i, j}$ ".

Hence by the definition of $R_{n+1, \zeta_{i, j}, \beta_{i, j}, k_{i, j}}$ we have

$$
M^{+} \models R_{n+1, \zeta_{i, j}, \beta_{i, j}, k_{i, j}}\left[a_{0}^{i}, \ldots, a_{n-1}^{i}, a_{k}^{j}\right] ;
$$


but $\beta_{i, j}<\beta$ and hence by the induction hypothesis

$$
\operatorname{rk}^{5}\left(\left\{a_{0}^{i}, \ldots, a_{n-1}^{j}, a_{k}^{j}\right\}, M^{+} ; \kappa\right) \leq \beta_{i, j} .
$$

So $F$ contradicts the choice of $\left\langle\left\langle a_{0}^{i}, \ldots, a_{n-1}^{i}\right\rangle: i<\kappa^{+}\right\rangle$, i.e. clause (a) ${ }^{-}$of Definition 1.1, case 5. 1.10

Claim 1.12. Let $B \subseteq{ }^{\omega} 2 \times{ }^{\omega} 2$ be a Borel or even analytic set and $\operatorname{Pr}_{\omega_{1}}(\lambda)$.

(1) If $B$ contains a $\lambda$-square then $B$ contains a perfect square.

(2) If $B$ contains a $(\lambda, \lambda)$-rectangle then $B$ contains a perfect rectangle.

(3) We can replace analytic by $\kappa$-Suslin if $\operatorname{Pr}_{\kappa^{+}}(\lambda ; \kappa)$. (This applies to $\Sigma_{2}^{1}$ sets which are $\aleph_{1}$-Suslin.)

Pr o o f. You can apply the results of Section 2 to prove 1.12; specifically, $2.1(1) \Rightarrow(2)$ proves parts (1), (2) and 2.5(1) proves part (3); those results of $\S 2$ say more, hence their proof should be clearer.

However, we give a proof of part (1) here for the reader who is going to read this section only. Suppose that $B \subseteq{ }^{\omega} 2 \times{ }^{\omega} 2$ is a Borel or even analytic set containing a $\lambda$-square. Let $T$ be a $(2,2, \omega)$-tree such that

$$
B=\left\{\left(\eta_{0}, \eta_{1}\right) \in{ }^{\omega} 2 \times{ }^{\omega} 2:\left(\exists \varrho \in{ }^{\omega} \omega\right)\left[\left(\eta_{0}, \eta_{1}, \varrho\right) \in \lim (T)\right]\right\},
$$

and let $\left\{\eta_{\alpha}: \alpha<\lambda\right\} \subseteq \omega_{2}$ be such that the square determined by it is contained in $B$ and $\alpha<\beta<\lambda \Rightarrow \eta_{\alpha} \neq \eta_{\beta}$. For $\alpha, \beta<\lambda$ let $F(\alpha, \beta) \in{ }^{\omega} \omega$ be such that $\left(\eta_{\alpha}, \eta_{\beta}, F(\alpha, \beta)\right) \in \lim (T)$. Define a model $M$ with universe $\lambda$ and vocabulary $\tau=\left\{R_{\nu_{0}, \nu_{1}, \nu}, Q_{\nu_{0}, \nu}: \nu_{0}, \nu_{1} \in{ }^{\omega>} 2\right.$ and $\left.\nu \in \omega>\omega\right\}$ where each $R_{\nu_{0}, \nu_{1}, \nu}$ is a binary predicate, $Q_{\nu_{0}, \nu}$ is a unary predicate and

$$
\begin{aligned}
Q_{\nu_{0}, \nu}^{M} & =\left\{\alpha<\lambda: \nu_{0} \triangleleft \eta_{\alpha} \& \nu \triangleleft F(\alpha, \alpha)\right\}, \\
R_{\nu_{0}, \nu_{1}, \nu}^{M} & =\left\{(\alpha, \beta) \in \lambda \times \lambda: \nu_{0} \triangleleft \eta_{\alpha} \& \nu_{1} \triangleleft \eta_{\beta} \& \nu \triangleleft F(\alpha, \beta)\right\} .
\end{aligned}
$$

By $\operatorname{Pr}_{\omega_{1}}(\lambda)$ we know that $\operatorname{rk}^{0}(M) \geq \omega_{1}$.

A pair $(u, h)$ is called an $n$-approximation if $u \subseteq{ }^{n} 2, h: u \times u \rightarrow{ }^{n} \omega$ and for every $\gamma<\omega_{1}$ there is $w \in[\lambda]^{*}$ such that:

$\left(\oplus_{1}\right) \quad u=\left\{\eta_{\alpha}\lceil n: \alpha \in w\}\right.$ and $\eta_{\alpha}\left\lceil n \neq \eta_{\beta}\lceil n\right.$ for distinct $\alpha, \beta \in w$,

$\left(\oplus_{2}\right) \quad \operatorname{rk}^{0}(w, M) \geq \gamma$,

$\left(\oplus_{3}\right) \quad F(\alpha, \beta)\left\lceil n=h\left(\eta_{\alpha}\left\lceil n, \eta_{\beta}\lceil n)\right.\right.\right.$ for $\alpha, \beta \in w$; hence

$$
M \models R_{\eta_{\alpha}\left\lceil n, \eta_{\beta}\left\lceil n, h\left(\eta _ { \alpha } \left\lceiln, \eta_{\beta}\lceil n)\right.\right.\right.\right.}[\alpha, \beta]
$$

for $\alpha, \beta \in w$.

Note that $(\{\langle\rangle\},\{((\langle\rangle,\langle\rangle),\langle\rangle)\})$ is a 0-approximation. Moreover,

$(*)_{0} \quad$ if $(u, h)$ is an $n$-approximation and $\nu^{*} \in u$ then there are $m>n$ and an $m$-approximation $\left(u^{+}, h^{+}\right)$such that:

(i) $\nu \in u \backslash\left\{\nu^{*}\right\} \Rightarrow\left(\exists ! \nu^{+}\right)\left(\nu \triangleleft \nu^{+} \in u^{+}\right)$, 
(ii) $\left(\exists^{! 2} \nu^{+}\right)\left(\nu^{*} \triangleleft \nu^{+} \in u^{+}\right)$(where $\exists^{! 2} x$ means "there are exactly 2 $x$ 's")

(iii) $\nu \in u^{+} \Rightarrow \nu\lceil n \in u$ and

(iv) if $\nu_{1}, \nu_{2} \in u^{+}$then

$$
\left[h \left(\nu _ { 1 } \left\lceiln , \nu _ { 2 } \lceil n ) \triangleleft h ^ { + } ( \nu _ { 1 } , \nu _ { 2 } ) \text { or } \left(\nu_{1}\left\lceil n=\nu_{2}\left\lceil n=\nu^{*} \& \nu_{1} \neq \nu_{2}\right)\right] .\right.\right.\right.\right.
$$

[Why? For each $\gamma<\omega_{1}$ choose $w_{\gamma}$ satisfying $\left(\oplus_{1}\right),\left(\oplus_{2}\right)$ and $\left(\oplus_{3}\right)$ for $\gamma+1$, now apply the definition of $\operatorname{rk}^{0}$ (if $w_{\gamma}=\left\{\alpha_{l}^{\gamma}: l<\left|w_{\gamma}\right|\right\}, \nu^{*} \triangleleft \eta_{\alpha_{k}^{\gamma}}$ and $k<\left|w_{\gamma}\right|$ we apply it to $\left.k\right)$ to get $w_{\gamma}^{+}=w_{\gamma} \cup\left\{\alpha_{\gamma}\right\}$ satisfying $\left(\oplus_{1}\right),\left(\oplus_{2}\right)$ and $\left(\oplus_{3}\right)$ for $\gamma$, then choose $m_{\gamma} \in(n, \omega)$ such that $\left\langle\eta_{\alpha} \mid m_{\gamma}: \alpha \in w_{\gamma}^{+}\right\rangle$is with no repetitions. Lastly, as there are only countably many possibilities for $\left\langle m_{\gamma},\left\{\eta_{\alpha}\left\lceil m_{\gamma}: \alpha \in w_{\gamma}^{+}\right\},\left\{\left(\eta_{\alpha}\left\lceil m_{\gamma}, \eta_{\beta}\left\lceil m_{\gamma}, F(\alpha, \beta)\left\lceil m_{\gamma}\right): \alpha, \beta \in w_{\gamma}^{+}\right\}\right\rangle\right.\right.\right.\right.$for $\gamma<\omega_{1}$, there is a value taken for uncountably many $\gamma$; let $\gamma^{*}$ be one of them. Choose $m=m_{\gamma^{*}}, u^{+}=\left\{\eta_{\alpha}\left\lceil m: \alpha \in w_{\gamma}^{+}\right\}\right.$and define $h^{+}$to satisfy $\left.\left(\oplus_{3}\right).\right]$

Repeating the procedure of $(*)_{0}|u|$ times we get

$(*)_{1} \quad$ if $u=\left\{\nu_{l}: l<k\right\} \subseteq{ }^{n} 2$ (no repetition) and $(u, h)$ is an $n$-approximation then there are $m, u^{+}=\left\{\nu_{l}^{+}: l<2 k\right\}$ and $h^{+}$such that $\left(u^{+}, h^{+}\right)$ is an $m$-approximation for some $m>n$ and:

(i) $\nu_{l} \triangleleft \nu_{2 l}^{+}, \nu_{l} \triangleleft \nu_{2 l+1}^{+}, \nu_{2 l}^{+} \neq \nu_{2 l+1}^{+}$,

(ii) if $l<k$ and $i<2$ then $h\left(\nu_{l}, \nu_{l}\right) \triangleleft h^{+}\left(\nu_{2 l+i}^{+}, \nu_{2 l+i}^{+}\right)$and

(iii) if $l_{1} \neq l_{2}, l_{1}, l_{2}<k, i, j<2$ then $h\left(\nu_{l_{1}}, \nu_{l_{2}}\right) \triangleleft h^{+}\left(\nu_{2 l_{1}+i}^{+}, \nu_{2 l_{2}+j}^{+}\right)$.

Consequently, we have

$(*)_{2} \quad$ there are sequences $\left\langle n_{i}: i<\omega\right\rangle \subseteq \omega$ and $\left\langle\left(u_{i}, h_{i}\right): i \in \omega\right\rangle$ such that $n_{i}<n_{i+1},\left(u_{i}, h_{i}\right)$ is an $n_{i}$-approximation and $\left(u_{i}, h_{i}\right),\left(u_{i+1}, h_{i+1}\right)$ are like $(u, h),\left(u^{+}, h^{+}\right)$of $(*)_{1}$.

Now, let $\left\langle n_{i}: i<\omega\right\rangle$ and $\left\langle\left(u_{i}, h_{i}\right): i \in \omega\right\rangle$ be as in $(*)_{2}$. Define

$$
\mathcal{P}=\left\{\eta \in{ }^{\omega} 2:(\forall i \in \omega)\left(\eta\left\lceil n_{i} \in u_{i}\right)\right\} .\right.
$$

By $(*)_{1}$ for $\left(u_{i+1}, h_{i+1}\right)$ we know that $\mathcal{P}$ is a perfect set. We claim that $\mathcal{P} \times \mathcal{P} \subseteq B$. Suppose that $\eta^{\prime}, \eta^{\prime \prime} \in \mathcal{P}$ and $\eta^{\prime} \neq \eta^{\prime \prime}$. Then $\eta^{\prime}\left\lceil n_{i(*)} \neq \eta^{\prime \prime}\left\lceil n_{i(*)}\right.\right.$ for some $i(*)<\omega$ and the sequence $\left\langle h_{i}\left(\eta^{\prime}\left\lceil n_{i}, \eta^{\prime \prime}\left\lceil n_{i}\right): i(*) \leq i<\omega\right\rangle\right.\right.$ is $\triangleleft$-increasing and (as $\left(u_{i}, h_{i}\right)$ are approximations) $\left(\eta^{\prime}\left\lceil n_{i}, \eta^{\prime \prime}\left\lceil n_{i}, h_{i}\left(\eta^{\prime}\left\lceil n_{i}\right.\right.\right.\right.\right.$, $\left.\eta^{\prime \prime}\left\lceil n_{i}\right)\right) \in T$ is increasing for $i \in[i(*), \omega)$. The case $\eta^{\prime}=\eta^{\prime \prime} \in \mathcal{P}$ is easier. The claim is proved.

TheOREM 1.13. Assume $\operatorname{NPr}_{\omega_{1}}(\lambda)$ and $\lambda \leq \mu=\mu^{\aleph_{0}}$. Then for some c.c.c. forcing notion $\mathbf{P},|\mathbf{P}|=\mu$ and $\vdash_{\mathbf{P}} " 2^{\aleph_{0}}=\mu$ " and in $\mathbf{V}^{\mathbf{P}}$ we have

(*) there is a Borel set $B \subseteq{ }^{\omega} 2 \times{ }^{\omega} 2$ such that:

(a) it contains a $\lambda$-square, i.e. there are pairwise distinct $\eta_{\alpha} \in{ }^{\omega} 2$ for $\alpha<\lambda$ such that $\left(\eta_{\alpha}, \eta_{\beta}\right) \in B$ for $\alpha, \beta<\lambda$, 
(b) let $\mathbf{V} \models \lambda^{\aleph_{0}}=\lambda_{1} ; B$ contains no $\lambda_{1}^{+}$-square, i.e. there are no $\eta_{\alpha} \in{ }^{\omega} 2\left(\right.$ for $\left.\alpha<\lambda_{1}^{+}\right)$such that $\left[\alpha \neq \beta \Rightarrow \eta_{\alpha} \neq \eta_{\beta}\right]$ and $\left(\eta_{\alpha}, \eta_{\beta}\right) \in$ $B$ for $\alpha, \beta<\lambda^{+}$,

(c) $B$ contains no perfect square.

Actually, $B$ is a countable union of closed sets.

Proof. Stage A. Clearly for some $\alpha(*)<\omega_{1}$ we have $\operatorname{NPr}_{\alpha(*)}^{1}(\lambda)$. Let $M$ be a model with universe $\lambda$ and a countable vocabulary such that $\operatorname{rk}^{1}(M)<\alpha(*)$, say with $<^{M}$ the usual order. Let functions $\varphi^{M}, k^{M}$ with domains

$$
[\lambda]^{*}=\{u: u \subseteq \lambda, u \text { is finite and } u \neq \emptyset\}
$$

be such that if $u=\left\{\alpha_{0}, \ldots, \alpha_{n-1}\right\} \in[\lambda]^{*}$ is increasing for definiteness and $\beta=\operatorname{rk}^{0}(u, M)(<\alpha(*))$ then $\varphi^{M}(u)$ is a quantifier free formula in the vocabulary of $M$ in the variables $x_{0}, \ldots, x_{n-1}$ for simplicity saying $x_{0}<x_{1}<\ldots<x_{n-1}, k^{M}(u)$ is a natural number $<n=|u|$ such that $\varphi^{M}(u), k^{M}(u)$ witness $\operatorname{rk}^{1}(u, M) \nsupseteq \beta+1$ (the same definition makes sense even if $\beta=-1)$. In particular,

$$
M \models \varphi^{M}(u)[\ldots, a, \ldots]_{a \in u} .
$$

We define the forcing notion $\mathbf{P}$. We can put the diagonal $\{(\eta, \eta): \eta \in$ $\left.\omega_{2}\right\}$ into $B$ so we can ignore it. We want to produce (in $\mathbf{V}^{\mathbf{P}}$ ) a Borel set $B=\bigcup_{n<\omega} B_{n}$, with each $B_{n}\left(\subseteq{ }^{\omega} 2 \times{ }^{\omega} 2\right)$ closed (in fact perfect), so $B_{n}$ is $\lim \left(T_{n}\right)$ for some $(2,2)$-tree $T_{n}, B_{0}$ is the diagonal, and $\bar{\eta}=\left\langle\eta_{\alpha}: \alpha<\mu\right\rangle$ as witnesses to $2^{\aleph_{0}} \geq \mu$ and such that $\left\{\eta_{\alpha}: \alpha<\lambda\right\}$ gives the desired square. So for some 2-place function $g$ from $\lambda$ to $\omega, \alpha \neq \beta \Rightarrow\left(\eta_{\alpha}, \eta_{\beta}\right) \in \lim \left(T_{g(\alpha, \beta)}\right)$, all this after we force. But we know that we shall have to use $M$ (by 1.12). In the forcing our problem will be to prove the c.c.c., which will be resolved by using $M$ (and rank) in the definition of the forcing. We shall have a function $f$ which puts the information on the rank into the trees to help in not having a perfect square. Specifically, the domain of $f$ is a subset of

$$
\left\{(u, h):(\exists l \in \omega)\left(u \in\left[{ }^{l} 2\right]^{*}\right) \text { and } h: u \times u \rightarrow \omega\right\}
$$

(the functions $h$ above are thought of as indexing the $B_{n}$ 's). The function $f$ will be such that for any distinct $\alpha_{0}, \ldots, \alpha_{n-1}<\lambda$, if $\left\langle\eta_{\alpha_{t}} \mid l: t<n\right\rangle$ are pairwise distinct, $u=\left\{\eta_{\alpha_{t}}\lceil l: t<n\}, h\left(\eta_{\alpha_{t}}\left\lceil l, \eta_{\alpha_{s}} \mid l\right)=g\left(\alpha_{t}, \alpha_{s}\right)\right.\right.$ and $(u, h) \in \operatorname{Dom}(f)$ then $\operatorname{rk}^{1}\left(\left\{\alpha_{l}: l<n\right\}, M\right)=f_{0}(u, h)$, and $f_{1}(u, h)$ is $\eta_{\alpha_{k}}\left\lceil l\right.$, where $k=k^{M}\left(\left\{\alpha_{t}: t<n\right\}\right), f_{2}(u, h)=\varphi^{M}\left(\left\{\alpha_{l}: l<n\right\}\right)$ writing the variable as $x_{\nu}, \nu \in u$, and $f(u, h)=\left(f_{0}(u, h), f_{1}(u, h), f_{2}(u, h)\right)$. (Note that $f$ is a way to say $\bigcup_{n} \lim \left(T_{n}\right)$ contains no perfect square; essentially it is equivalent to fixing an appropriate rank.) All this was to motivate the definition of the forcing notion $\mathbf{P}$.

A condition $p$ ( of $\mathbf{P}$ ) is an approximation to all this; it consists of: 
(1) $u^{p}=u[p]$, a finite subset of $\mu$.

(2) $n^{p}=n[p]<\omega$ and $\eta_{\alpha}^{p}=\eta_{\alpha}[p] \in{ }^{n[p]} 2$ for $\alpha \in u[p]$ such that $\alpha \neq \beta \Rightarrow \eta_{\alpha}^{p} \neq \eta_{\beta}^{p}$.

(3) $\bar{m}^{p}=\left\langle m_{l}^{p}: l \leq n^{p}\right\rangle$ is a strictly increasing sequence of natural numbers with last element $m_{n[p]}^{p}=m^{p}=m[p]$ and for $m<m[p]$, we have $t_{m}^{p}=t_{m}[p] \subseteq \bigcup_{l \leq n[p]}\left({ }^{l} 2 \times{ }^{l} 2\right)$ which is downward closed (i.e., $\left(\nu_{0}, \nu_{1}\right) \in$ $t_{m}^{p} \cap\left({ }^{l} 2 \times{ }^{l} 2\right) \& k<l \Rightarrow\left(\nu_{0}, \nu_{1}\right)\left\lceil k=\left(\nu_{0}\left\lceil k, \nu_{1}\lceil k) \in t_{m}^{p}\right)\right.\right.$; also $(\langle\rangle,\langle\rangle) \in t_{m}^{p}$ and defining $\triangleleft$ naturally we have:

$$
\begin{aligned}
& \text { if }\left(\eta_{0}, \eta_{1}\right) \in t_{m}^{p} \cap\left({ }^{l} 2 \times{ }^{l} 2\right) \text { and } l<n^{p} \\
& \text { then }\left(\exists \nu_{0}, \nu_{1}\right)\left[\left(\eta_{0}, \eta_{1}\right) \triangleleft\left(\nu_{0}, \nu_{1}\right) \in t_{m}^{p} \cap\left({ }^{l+1} 2 \times{ }^{l+1} 2\right)\right] .
\end{aligned}
$$

(4) A function $f^{p}=f[p]$ whose domain is a subset of

$\left\{(u, h)\right.$ : for some $l \leq n[p], u \subseteq{ }^{l} 2,|u| \geq 1, h$ is a 2 -place function from $u$ to $m[p]$ such that $[\eta \in u \Rightarrow h(\eta, \eta)=0]$ and $\left.\left[\eta, \nu \in u \Rightarrow(\eta, \nu) \in t_{h(\eta, \nu)}^{p}\right]\right\}$

and $f^{p}$ is such that

$$
f^{p}(u, h)=\left(f_{0}^{p}(u, h), f_{1}^{p}(u, h), f_{2}^{p}(u, h)\right) \in[-1, \alpha(*)) \times u \times L_{\omega, \omega}(\tau(M)) .
$$

(5) A function $g=g^{p}$ with domain $\left\{(\alpha, \beta): \alpha, \beta\right.$ from $\left.u^{p} \cap \lambda\right\}$ such that $g(\alpha, \alpha)=0$ and $\alpha \neq \beta \Rightarrow\left[0<g(\alpha, \beta)<m^{p}\right.$ and $\left(\eta_{\alpha}^{p}, \eta_{\beta}^{p}\right) \in t_{g(\alpha, \beta)}^{p} \cap$ $\left.\left.{ }^{n(p)} 2 \times{ }^{n(p)} 2\right)\right]$.

(6) $t_{0}^{p}=\left\{(\eta, \eta): \eta \in n^{p} \geq 2\right\}$.

(7) If $u \subseteq{ }^{l} 2,|u| \geq 1, f^{p}(u, h)=\left(\beta^{*}, \varrho^{*}, \varphi^{*}\right)$, and $l<l(*) \leq n^{p}, e_{i}$ are functions with domain $u$ (for $i=0,1)$ such that $(\forall \varrho)\left[\varrho \in u \Rightarrow \varrho \triangleleft e_{i}(\varrho) \in\right.$ $\left.\left.{ }^{l(*)} 2\right]\right)$ and $(\forall \varrho \in u)\left[e_{0}(\varrho)=e_{1}(\varrho) \Leftrightarrow \varrho \neq \varrho^{*}\right], u^{\prime}=\operatorname{Rang}\left(e_{0}\lceil u) \cup \operatorname{Rang}\left(e_{1}\lceil u)\right.\right.$, and $h(\eta, \nu)=h^{\prime}\left(e_{i}(\eta), e_{i}(\nu)\right)$ for $\eta \neq \nu$ in $u$ and $f^{p}\left(u^{\prime}, h^{\prime}\right)=\left(\beta^{\prime}, \varrho^{\prime}, \varphi^{\prime}\right)$ (so is well defined) then $\beta^{\prime}<\beta^{*}$.

(8) If $l \leq n^{p}, w \subseteq u^{p} \cap \lambda$ is nonempty, the sequence $\left\langle\eta_{\alpha}^{p}\lceil l: \alpha \in w\rangle\right.$ is with no repetitions and $h$ is defined by $h\left(\eta_{\alpha}^{p}\left\lceil l, \eta_{\beta}^{p} \uparrow l\right)=g^{p}(\alpha, \beta)<m_{l}^{p}\right.$ for $\alpha \neq \beta$ from $w\left(\right.$ and $h\left(\eta_{\alpha}^{p}\left\lceil l, \eta_{\alpha}^{p}\lceil l)=0\right.\right.$ ) and $u=\left\{\eta_{\alpha}^{p}\lceil l: \alpha \in w\}\right.$ then $f^{p}(u, h)$ is well defined, $f_{2}^{p}(u, h)=\varphi^{M}(w), f_{1}^{p}(u, h)=\eta_{\alpha}^{p}\left\lceil l\right.$ where $\alpha$ is the $k^{M}(w)$ th member of $w$ and $f_{0}^{p}(u, h)=\operatorname{rk}^{1}(w, M)$; of course in $f_{2}^{p}(u, h)=\varphi^{M}(w)$ the variable $x_{\nu}$ in $f_{2}^{p}(u, h)$ corresponds to $x_{|\alpha \cap w|}$ if $\eta_{\alpha}\lceil l=\nu$ (see last clause of $\oplus_{p}$ below).

(9) If $(u, h) \in \operatorname{Dom}\left(f^{p}\right)$ then for some $w$ and $l, f^{p}(u, h)$ is as in (8).

(10) If $\eta_{1} \neq \eta_{2}$ are in ${ }^{l} 2$ with $l \leq n^{p}$ and $\left(\eta_{1}, \eta_{2}\right) \in t_{m}^{p}$ with $0<m<m^{p}$ then for some $\alpha_{1} \neq \alpha_{2}$ from $u^{p} \cap \lambda$ we have $g^{p}\left(\alpha_{1}, \alpha_{2}\right)=m$ and $\eta_{1} \unlhd \eta_{\alpha_{1}}^{p}$, $\eta_{2} \unlhd \eta_{\alpha_{2}}^{p}$.

The order is the natural one (including the following requirements: if $p \leq q$ then $n^{p} \leq n^{q}, m^{p} \leq m^{q}, \bar{m}^{p}=\bar{m}^{q} \uparrow\left(n^{p}+1\right), u^{p} \subseteq u^{q}, \eta_{\alpha}^{q}\left\lceil n^{p}=\eta_{\alpha}^{p}\right.$ 
for $\alpha \in u^{p}, t_{m}^{p}=t_{m}^{q} \cap \bigcup_{l \leq n[p]}\left({ }^{l} 2 \times{ }^{l} 2\right)$ for $m<m^{p}, g^{p}=g^{q}\left\lceil u^{p}\right.$ and $f^{p}=$ $f^{q} \uparrow\left\{(u, h) \in \operatorname{Dom}\left(f^{q}\right): u \subseteq n^{p} \geq 2\right\}$, so if $(u, h) \notin \operatorname{Dom}\left(f^{p}\right)$ with $u \subseteq n^{p} \geq 2$ then $\left.(u, h) \notin \operatorname{Dom}\left(f^{q}\right)\right)$.

Explanation. The function $f^{p}$ of a condition $p \in \mathbf{P}$ carries no additional information. It is determined by the functions $g^{p}, \varphi^{M}, k^{M}$ and the rank. Conditions (8), (9) are to say that

$\oplus_{p} \quad$ if $w_{0}, w_{1} \subseteq \lambda \cap u^{p}, l \leq n^{p}, u=\left\{\eta_{\alpha}^{p}\left\lceil l: \alpha \in w_{0}\right\}=\left\{\eta_{\alpha}^{p}\left\lceil l: \alpha \in w_{1}\right\}\right.\right.$ (no repetitions) are nonempty and $h: u \times u \rightarrow m^{p}$ is such that [if either $\alpha, \beta \in w_{0}$ or $\alpha, \beta \in w_{1}$ then $h\left(\eta_{\alpha}^{p}\left\lceil l, \eta_{\beta}^{p}\lceil l)=g^{p}(\alpha, \beta)\right]\right.$, then $\operatorname{rk}^{1}\left(w_{0}, M\right)=\operatorname{rk}^{1}\left(w_{1}, M\right), \varphi^{M}\left(w_{0}\right)=\varphi^{M}\left(w_{1}\right), k^{M}\left(w_{0}\right)=k^{M}\left(w_{1}\right)$, and if $\alpha_{i}, \beta_{i} \in w_{i}$ for $i=0,1$ and $\eta_{\alpha_{0}} \uparrow l=\eta_{\alpha_{1}} \uparrow l, \eta_{\beta_{0}} \uparrow l=\eta_{\beta_{1}} \uparrow l$ then $\alpha_{0}<\alpha_{1} \Leftrightarrow \beta_{0}<\beta_{1}$.

Moreover, condition (7) gives no additional restriction unless $f_{0}^{p}(u, h)=$ -1. Indeed, suppose that $u \subseteq{ }^{l} 2,|u| \geq 1, l<l(*) \leq n^{p}, e_{i}: u \rightarrow{ }^{l(*)} 2, h$, $\varrho^{*} \in u, u^{\prime}$ and $h^{\prime}$ are as there and $f_{0}^{p}(u, h) \geq 0$. As $f^{p}\left(u^{\prime}, h^{\prime}\right)$ is defined we find $w \subseteq \lambda \cap u^{p}$ and $\alpha_{0}, \alpha_{1} \in w\left(\alpha_{0} \neq \alpha_{1}\right)$ such that $u^{\prime}=\left\{\eta_{\alpha}^{p}\lceil l(*): \alpha \in w\}\right.$, $h^{\prime}\left(\eta_{\alpha}^{p}\left\lceil l(*), \eta_{\beta}^{p}\lceil l(*))=g^{p}(\alpha, \beta)<m_{l}^{p}\right.\right.$ and $e_{i}\left(\varrho^{*}\right)=\eta_{\alpha_{i}}^{p}\lceil l(*)($ for $i=0,1)$. Looking at $w \backslash\left\{\alpha_{0}\right\}, w \backslash\left\{\alpha_{1}\right\}$ and $(u, h)$ we see that

$$
\begin{gathered}
\alpha_{0}=k^{M}\left(w \backslash\left\{\alpha_{1}\right\}\right), \quad \varphi^{M}\left(w \backslash\left\{\alpha_{0}\right\}\right)=\varphi^{M}\left(w \backslash\left\{\alpha_{1}\right\}\right), \\
\operatorname{rk}^{1}\left(w \backslash\left\{\alpha_{1}\right\}, M\right)=f_{0}^{p}(u, h) \geq 0 .
\end{gathered}
$$

By the definition of the rank and the choice of $\varphi^{M}, k^{M}$ we get $\operatorname{rk}^{1}(w, M)=$ $f_{0}^{p}(u, h)$ and hence $f_{0}^{p}\left(u^{\prime}, h^{\prime}\right)<f_{0}^{p}(u, h)$.

If $f_{0}^{p}(u, h)=-1$ then clause (7) says that there are no respective $e_{0}, e_{1}$ introducing a ramification.

Stage B. $\mathbf{P}$ satisfies the c.c.c. Let $p^{i} \in \mathbf{P}$ for $i<\omega_{1}$; let $u\left[p^{i}\right]=\left\{a_{l}^{i}\right.$ : $\left.l<\left|u\left[p^{i}\right]\right|\right\}$ increasing, so with no repetition. We can assume that $\left|u\left[p^{i}\right]\right|$ does not depend on $i$, nor do $n\left[p^{i}\right], \eta_{a_{1}^{i}}^{p^{i}}, \bar{m}^{p^{i}},\left\langle t_{m}^{p^{i}}: m<m^{p^{i}}\right\rangle, g^{p^{i}}\left(a_{l_{1}}^{i}, a_{l_{2}}^{i}\right)$, $f\left[p^{i}\right]$, and for a nonempty $v \subseteq\left|u\left[p^{i}\right]\right|$ such that $\bigwedge_{l \in v} a_{l}^{i}<\lambda, \operatorname{rk}^{1}\left(\left\{a_{l}^{i}:\right.\right.$ $l \in v\}, M), \varphi^{M}\left(\left\{a_{l}^{i}: l \in v\right\}\right), k^{M}\left(\left\{a_{l}^{i}: l \in v\right\}\right)$ and the truth value of $a_{l}^{i} \geq \lambda$ does not depend on $i$. Note that writing $\varphi[w]$ we always assume that $\varphi$ carries information on the order of $w$.

Also by the $\Delta$-system argument, without loss of generality,

$$
a_{l_{1}}^{i^{1}}=a_{l_{2}}^{i^{2}} \& i^{1} \neq i^{2} \Rightarrow l_{1}=l_{2} \& \bigwedge_{i, j} a_{l_{1}}^{i}=a_{l_{2}}^{j} .
$$

We shall show that $p^{0}, p^{1}$ are compatible by defining a common upper bound $q$ :

(i) $n^{q}=n\left[p^{i}\right]+1$.

(ii) $u^{q}=\left\{a_{l}^{i}: l<\left|u\left[p^{i}\right]\right|, i<2\right\}$. 
(iii) $\eta_{a_{l}^{i}}^{q}$ is: $\eta_{a_{l}^{0}}^{p^{0}} \wedge\langle 0\rangle$ if $i=0, \eta_{a_{l}^{0}}^{p^{0}} \wedge\langle 1\rangle=\eta_{a_{l}^{1}}^{p^{1}} \wedge\langle 1\rangle$ if $i=1$ and $a_{l}^{0} \neq a_{l}^{1}$.

(iv) $m[q]=m\left[p^{0}\right]+2 \times\left|\lambda \cap u\left[p^{0}\right] \backslash u\left[p^{1}\right]\right|^{2}, \bar{m}^{q}=\bar{m}^{p^{0} \wedge}\langle m[q]\rangle$.

(v) $g^{q} \supseteq g^{p_{0}} \cup g^{p_{1}}$ is such that $g^{q}$ assigns new (i.e. in $\left[m^{p}, m^{q}\right)$ ) distinct values to "new" pairs $(\alpha, \beta)$ with $\alpha \neq \beta$, i.e. pairs from $(\lambda \times \lambda) \cap\left(u^{q} \times u^{q}\right) \backslash$ $u^{p^{0}} \times u^{p^{0}} \backslash u^{p^{1}} \times u^{p^{1}}$.

(vi) The trees $t_{m}^{q}$ (for $m<m[q]$ ) are defined as follows. If $m=0$ see clause (6); if $m<m\left[p^{0}\right], m>0$ then

$$
\begin{array}{r}
t_{m}^{q}=t_{m}^{p^{0}} \cup\left\{\left(\eta_{a_{l_{1}}^{\varepsilon}}^{q}, \eta_{a_{l_{2}}^{\varepsilon}}^{q}\right): \varepsilon \in\{0,1\} \text { and distinct } l_{1}, l_{2}<\left|u\left[p^{0}\right]\right|\right. \\
\text { satisfying } \left.g^{p^{0}}\left(a_{l_{1}}^{0}, a_{l_{2}}^{0}\right)=m\right\} ;
\end{array}
$$

and if $m \in\left[m\left[p^{0}\right], m[q]\right), m=g^{q}(\alpha, \beta)$ and $\alpha \neq \beta$ then

$$
t_{m}^{q}=\left\{\left(\eta_{\alpha}^{q} \backslash l, \eta_{\beta}^{q}\lceil l): l \leq n^{q}\right\} .\right.
$$

(vii) If $m \in\left[m\left[p^{0}\right], m[q]\right)$ then $m=g^{q}(\alpha, \beta)$ for one and only one pair $(\alpha, \beta)$, and for this pair we have $\alpha \neq \beta,\{\alpha, \beta\} \nsubseteq u\left[p^{0}\right]$ and $\{\alpha, \beta\} \nsubseteq u\left[p^{1}\right]$.

(viii) The function $f^{q}$ is determined by $g^{p}$ and clauses (8), (9) of stage A.

Of course we have to check that no contradiction appears when we define $f^{q}$ (i.e. we have to check $\oplus_{q}$ of the Explanation inside stage A for $q)$. So suppose that $w_{0}, w_{1} \subseteq \lambda \cap u[q], l \leq n[q], u, h$ are as in $\oplus_{q}$. If $w_{0} \subseteq u\left[p^{i}\right]$ (for some $i<2$ ) then $g^{q}(\alpha, \beta)<m\left[p^{0}\right]$ for $\alpha, \beta \in w_{0}$ and therefore $g^{q}\left[w_{1} \times w_{1}\right] \subseteq m\left[p^{0}\right]$. Consequently, either $w_{1} \subseteq u\left[p^{0}\right]$ or $w_{1} \subseteq u\left[p^{1}\right]$. If $l=n^{q}$ then necessarily $w_{0}=w_{1}$ so we have nothing to prove. If $l<n^{q}$ then $(u, h) \in \operatorname{Dom}\left(f^{p^{0}}\right)$ (and $\left.f^{p^{0}}=f^{p^{1}}\right)$ and clause 8 of stage A applies.

If $w_{0}$ is contained neither in $u\left[p^{0}\right]$ nor in $u\left[p^{1}\right]$ then $g^{q}(\alpha, \beta) \in\left[m\left[p^{0}\right], m[q]\right)$ for some $\alpha, \beta \in w_{0}$, hence $l=n^{q}$, and so since $\left\{\eta_{\alpha}^{q}\left\lceil l: \alpha \in w_{0}\right\}=\left\{\eta_{\alpha}^{q}\lceil l\right.\right.$ : $\left.\alpha \in w_{1}\right\}$, clearly $w_{0}=w_{1}$, so we are done.

Next we have to check condition (7). As we remarked (in the Explanation inside stage A) we have to consider cases of $(u, h)$ such that $f^{q}(u, h)=-1$ only. Suppose that $u, l<l(*) \leq n^{q}, e_{i}, h, \varrho^{*} \in u, u^{\prime}$ and $h^{\prime}$ are as in (7) (and $f^{q}(u, h)=-1$ ). Let $w \subseteq u[q] \cap \lambda$ and $\alpha_{0}, \alpha_{1} \in w$ be such that $u^{\prime}=\left\{\eta_{\alpha}^{q}\lceil l(*): \alpha \in w\}, e_{i}\left(\varrho^{*}\right)=\eta_{\alpha_{i}}^{q} \backslash l(*)\right.$ (for $\left.i=0,1\right)$. If $w \subseteq u\left[p^{i}\right]$ for some $i<2$ then we can apply clause (7) for $p^{i}$ and get a contradiction (if $l(*)=n^{q}$ then $\left\{\eta_{\alpha}^{q}\left\lceil n^{p}: \alpha \in w\right\}\right.$ are already distinct). Since $\alpha \in w \backslash\left\{\alpha_{0}, \alpha_{1}\right\}$ implies $g^{q}\left(\alpha, \alpha_{0}\right)=g^{q}\left(\alpha, \alpha_{1}\right)$ (by the relation between $h$ and $h^{\prime}$ ) we are left with the case $w \backslash\left\{\alpha_{0}, \alpha_{1}\right\} \subseteq u\left[p^{0}\right] \cap u\left[p^{1}\right], \alpha_{0} \in u\left[p^{0}\right] \backslash u\left[p^{1}\right], \alpha_{1} \in$ $u\left[p^{1}\right] \backslash u\left[p^{0}\right]$ (or conversely). Then necessarily $\alpha_{0}=a_{k_{0}}^{0}$ and $\alpha_{1}=a_{k_{1}}^{1}$ for some $k_{0}, k_{1} \in\left[0,\left|u\left[p^{0}\right]\right|\right)$. Now $k_{1}=k^{M}\left(w \backslash\left\{\alpha_{0}\right\}\right)=k^{M}\left(w \backslash\left\{\alpha_{1}\right\}\right)=k_{0}$ by the requirements in condition (7). We see that for each $i<\omega_{1}$,

$$
M \models \varphi^{M}\left(w \backslash\left\{\alpha_{0}\right\}\right)\left[w \backslash\left\{\alpha_{0}, \alpha_{1}\right\} \cup\left\{a_{k_{1}}^{i}\right\}\right],
$$


and this contradicts the fact that $\varphi^{M}\left(w \backslash\left\{\alpha_{0}\right\}\right)$ and $\alpha_{1}$ witness $\operatorname{rk}^{1}(w \backslash$ $\left.\left\{\alpha_{0}\right\}, M\right)=-1$.

Stage C. $|\mathbf{P}|=\mu$ hence $\vdash_{\mathbf{P}}$ " $2^{\aleph_{0}} \leq \mu$ ". We shall get equality by clause $(\gamma)$ at stage $\mathrm{E}$ below.

Stage D. The following subsets of $\mathbf{P}$ are dense (for $m, n<\omega, \alpha<\mu)$ :

$$
\begin{gathered}
\mathcal{I}_{m}^{1}=\{p \in \mathbf{P}: m[p] \geq m\}, \quad \mathcal{I}_{n}^{2}=\left\{p \in \mathbf{P}: n^{p} \geq n\right\}, \\
\mathcal{I}_{\alpha}^{3}=\{p \in \mathbf{P}: \alpha \in u[p]\} .
\end{gathered}
$$

Proof. Let $p \in \mathbf{P}$ and $\alpha_{0} \in \mu \backslash u[p]$ be given. We shall find $q$ with $p \leq q \in \mathcal{I}_{m[p]+1}^{1} \cap \mathcal{I}_{n[p]+1}^{2} \cap \mathcal{I}_{\alpha_{0}}^{3}$; this clearly suffices. We may assume that $u[p] \neq \emptyset$ and $\alpha_{0}<\lambda$. We make the following definitions:

(a) $n^{q}=n^{p}+1, m^{q}=m^{p}+2 \cdot|\lambda \cap u[p]|, \bar{m}^{q}=\bar{m}^{p \wedge}\left\langle m^{q}\right\rangle, u^{q}=u^{p} \cup\left\{\alpha_{0}\right\}$.

(b) For $\alpha \in u^{p}$ we let $\eta_{\alpha}^{q}=\eta_{\alpha}^{p} \wedge\langle 0\rangle$, and $\eta_{\alpha_{0}}^{q} \in{ }^{\left(n^{p}+1\right)} 2$ is the sequence constantly 1 .

(c) $g^{q}$ is any 2-place function from $u^{q} \cap \lambda$ to $m^{q}$ extending $g^{p}$ such that $g^{q}(\alpha, \alpha)=0, g^{q}(\alpha, \beta) \neq 0$ for $\alpha \neq \beta$ and

$$
\begin{aligned}
g^{q}(\alpha, \beta)=g^{q}\left(\alpha^{\prime}, \beta^{\prime}\right) \&(\alpha, \beta) & \neq\left(\alpha^{\prime}, \beta^{\prime}\right) \\
& \Rightarrow(\alpha, \beta) \in u^{p} \times u^{p} \&\left(\alpha^{\prime}, \beta^{\prime}\right) \in u^{p} \times u^{p} .
\end{aligned}
$$

(d) $t_{m}^{q}$ is defined as follows:

$(\alpha)$ if $m<m^{p}, m \neq 0$ then $t_{m}^{q}=t_{m}^{p} \cup\left\{\left(\eta_{0} \wedge\langle 0\rangle, \eta_{1} \wedge\langle 0\rangle\right):\left(\eta_{0}, \eta_{1}\right) \in\right.$ $\left.t_{m}^{p} \cap\left({ }^{n[p]} 2 \times{ }^{n[p]} 2\right)\right\}$,

$(\beta)$ if $m \in\left[m^{p}, m^{q}\right), m=g^{q}(\alpha, \beta), \alpha \neq \beta$ then $t_{m}^{q}=\left\{\left(\eta_{\alpha}^{q}\left\lceil l, \eta_{\beta}^{q}\lceil l)\right.\right.\right.$ : $\left.l \leq n^{q}\right\}$.

(e) $f^{q}$ extends $f^{p}$ and satisfies (7)-(9) of stage A (note that $f^{q}$ is determined by $\left.g^{q}\right)$.

Now check (similarly to stage B).

Stage E. We define some $\mathbf{P}$-names:

(a) $\eta_{\alpha}=\bigcup\left\{\eta_{\alpha}^{p}: p \in G_{\mathbf{P}}\right\}$ for $\alpha<\lambda$,

(b) $\underset{\sim}{T} m=\bigcup\left\{t_{m}^{p}: p \in G_{\mathbf{P}}\right\}$ for $m<\omega$,

(c) $\underset{q}{g}=\bigcup\left\{g^{p}: p \in G_{\mathbf{P}}\right\}$.

Clearly it is forced $\left(\Vdash_{\mathbf{P}}\right)$ that:

$(\alpha) g$ is a function from $\{(\alpha, \beta): \alpha, \beta<\lambda\}$ to $\omega$. [Why? Because $\mathcal{I}_{\alpha}^{3}$ are dense subsets of $\mathbf{P}$ and by clause (5) of stage A.]

( $\beta) \eta_{\alpha} \in{ }^{\omega} 2$. [Why? Because both $\mathcal{I}_{n}^{2}$ and $\mathcal{I}_{\alpha}^{3}$ are dense subsets of $\mathbf{P}$.]

$(\gamma) \widetilde{\eta}_{\alpha} \neq \eta_{\beta}$ for $\alpha \neq \beta(<\mu)$. [Why? By clause (2) of the definition of $p \in \mathbf{P}$.] 


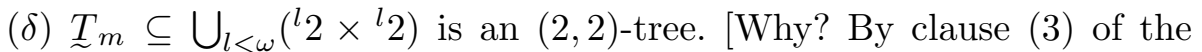
definition of $p \in \mathbf{P}$ and density of $\mathcal{I}_{m}^{1}, \mathcal{I}_{n}^{2}$.]

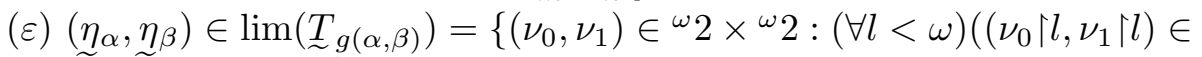
$\underset{\sim}{T} g(\alpha, \beta)) \widetilde{f}(\widetilde{f o r} \alpha, \beta<\lambda)$. [Why? By clause (5) of the definition of $p \in \mathbf{P}$ and $(\widetilde{\beta})+(\delta)$ above.]

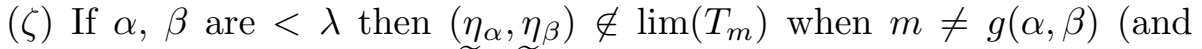
$m<\omega)$. [Why? By clauses $(2)+(\widetilde{10})$ of the definition of $\mathbf{P}$ if $m \neq 0$, and clause (5) if $m=0$.]

Note that by $(\varepsilon)$ above, the Borel set $\underset{\sim}{B}=\bigcup_{m<\omega} \lim (\underset{\sim}{T} m) \subseteq{ }^{\omega} 2 \times{ }^{\omega} 2$ satisfies requirement $(*)($ a) of the conclusion of 1.13 . Moreover, by $(\gamma)$ we have $\Vdash_{\mathbf{P}} " 2^{\aleph_{0}} \geq \mu$ ", completing stage C (i.e. $\vdash_{\mathbf{P}} " 2^{\aleph_{0}}=\mu$ ").

Stage F. We want to show $(*)(\mathrm{c})$ of the conclusion of 1.13. Let $\mathbf{P}_{\lambda}=$ $\{p \in \mathbf{P}: u[p] \subseteq \lambda\}$. Clearly $\mathbf{P}_{\lambda} \lessdot \mathbf{P}$. Moreover, $\underset{\sim}{g} \underset{\sim}{\underset{\sim}{T}}, \underset{\sim}{B}$ are $\mathbf{P}_{\lambda}$-names. Since " $\underset{\sim}{B}$ contains a perfect square" is a $\Sigma_{2}^{1}$-formula, so absolute, it is enough to prove that in $\mathbf{V}^{\mathbf{P}_{\lambda}}$ the set $\underset{\sim}{B}$ contains no perfect square.

Suppose that a $\mathbf{P}_{\lambda}$-name $\underset{\sim}{T}$ for a perfect tree and a condition $p \in \mathbf{P}_{\lambda}$ are such that

$$
p \Vdash_{\mathbf{P}_{\lambda}} " \lim (\underset{\sim}{T}) \times \lim (\underset{\sim}{T}) \subseteq \underset{\sim}{B} " .
$$

We then have (a name for) a function $\underset{\sim}{m}: \lim (\underset{\sim}{T}) \times \lim (\underset{\sim}{T}) \rightarrow \omega$ such that

$$
p \Vdash_{\mathbf{P}_{\lambda}} \text { "if } \eta_{0}, \eta_{1} \in \lim (\underset{\sim}{T}) \text { then }\left(\eta_{0}, \eta_{1}\right) \in \underset{\sim}{T} \underset{\sim}{m}\left(\eta_{0}, \eta_{1}\right) \text { ". }
$$

By shrinking the tree $\underset{\sim}{T}$ we may assume that $p$ forces $\left(\|_{\mathbf{P}_{\lambda}}\right)$ the following:

$$
\begin{aligned}
& \text { "if } \eta_{0}, \eta_{1}, \eta_{0}^{\prime}, \eta_{1}^{\prime} \in \lim (\underset{\sim}{T}) \text { and } \eta_{0}\left\lceil l=\eta_{0}^{\prime} \uparrow l \neq \eta_{1} \uparrow l=\eta_{1}^{\prime} \uparrow l\right. \\
& \text { then } \underset{\sim}{m}\left(\eta_{0}, \eta_{1}\right)=\underset{\sim}{m}\left(\eta_{0}^{\prime}, \eta_{1}^{\prime}\right) \text { ". }
\end{aligned}
$$

Consequently, we may think of $\underset{\sim}{m}$ as a function from $\underset{\sim}{T} \times \underset{\sim}{T}$ to $\omega$ (with the convention that if $\nu_{0}, \nu_{1} \in \underset{\sim}{T}$ are $\triangleleft$-comparable then $\underset{\sim}{m}\left(\nu_{0}, \nu_{1}\right)=0$ and $\eta^{\wedge}\langle l\rangle \triangleleft \nu_{l} \in \underset{\sim}{T} \Rightarrow \underset{\sim}{m}\left(\eta^{\wedge}\langle l\rangle, \eta^{\wedge}\langle 1-l\rangle\right)=\underset{\sim}{m}\left(\nu_{l}, \nu_{1-l}\right)$ and if $\lg \left(\nu_{1}\right)=\lg \left(\nu_{2}\right)$ then $\left.\left(\nu_{1}, \nu_{2}\right) \in T_{m\left(\nu_{1}, \nu_{2}\right)}\right)$.

Choose an increasing sequence $\left\langle n_{i}: i \in \omega\right\rangle$ of natural numbers and sequences $\left\langle p_{i}: i \in \omega\right\rangle \subseteq \mathbf{P}_{\lambda},\left\langle\left(t_{i}, m_{i}\right): i \in \omega\right\rangle$ such that:

(1) $p_{0} \leq p_{1} \leq \ldots \leq p_{i} \leq p_{i+1} \leq \ldots$,

(2) $t_{i} \subseteq{ }^{n_{i}} \geq 2$ is a perfect tree (i.e. $\left[\eta \triangleleft \nu \in t_{i} \cap{ }^{n_{i}} \geq 2 \Rightarrow \eta \in t_{i}\right],\langle\rangle \in t_{0}$, $\left.\left[\eta \in{ }^{n_{i}>} 2 \cap t_{i} \Rightarrow \bigvee_{l<2} \eta^{\wedge}\langle l\rangle \in t_{i}\right]\right)$ and $m_{i}:\left(t_{i} \cap{ }^{n_{i}} 2\right)^{2} \rightarrow \omega$,

(3) $t_{i} \subseteq t_{i+1}$ is an end extension (i.e. $\left.t_{i}=\left({ }^{n_{i} \geq 2}\right) \cap t_{i+1}\right)$ such that each node from $t_{i} \cap{ }^{n_{i}} 2$ ramifies in $t_{i+1}$ (i.e. has $\triangleleft$-incomparable extensions),

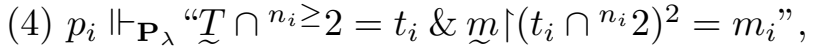

(5) $n\left[p_{i}\right]>n_{i}$ and $m\left[p_{i}\right]>\max \operatorname{Rang}\left(m_{i}\right)$. 
Since $p_{i} \Vdash_{\mathbf{P}_{\lambda}}$ " $\left(\nu_{0}, \nu_{1}\right) \in \underset{\sim}{T} m_{i}\left(\nu_{0}, \nu_{1}\right)$ " for $\nu_{0}, \nu_{1} \in t_{i} \cap{ }^{n_{i}} 2$ we easily see (by clause (8) of the definition of $\mathbf{P}$, stage A) that $\left(t_{i} \cap{ }^{n_{i}} 2, m_{i}\right) \in \operatorname{Dom}\left(f^{p_{i}}\right)$. By clause (7) (of the definition of $\mathbf{P}$ ) (and 1.2(2) + clause (8) of the definition of $\mathbf{P}$ ) we deduce that

$$
f_{0}^{p_{i+1}}\left(t_{i+1} \cap{ }^{n_{i+1}} 2, m_{i+1}\right)<f_{0}^{p_{i}}\left(t_{i} \cap{ }^{n_{i}} 2, m_{i}\right)
$$

for each $i<\omega$, and this gives a contradiction (to the ordinals being well ordered).

Stage G. To prove $(*)($ b) of Theorem 1.13 we may assume that $\mathbf{V} \models$ " $\lambda^{\aleph_{0}}=\lambda_{1}<\mu$ ". Let $\mathbf{P}_{\lambda_{1}}=\left\{p \in \mathbf{P}: u[p] \subseteq \lambda_{1}\right\} \lessdot \mathbf{P}$. Note that the rest of the forcing (i.e. $\mathbf{P} / \mathbf{P}_{\lambda_{1}}$ ) is the forcing notion for adding $\mu$ Cohen reals so for $v \subseteq \mu \backslash \lambda_{1}$ the forcing notion $\mathbf{P}_{v}$ is naturally defined, as also is $\mathbf{P}_{\lambda_{1} \cup v}$. By stages $\mathrm{C}, \mathrm{E}$ we know that $\mathbf{V}^{\mathbf{P}_{\lambda_{1}}}=" 2^{\aleph_{0}}=\lambda_{1}$ " and by stage F we have $\mathbf{V}^{\mathbf{P}_{\lambda_{1}}}=$ "the Borel set $\underset{\sim}{B}$ does not contain a perfect square". Suppose that after adding $\mu$ Cohen reals (over $\mathbf{V}^{\mathbf{P}_{\lambda_{1}}}$ ) we have a $\lambda_{1}^{+}$-square contained in $\underset{\sim}{B}$. We have $\lambda_{1}^{+}$-branches $\varrho_{\alpha}\left(\alpha<\lambda_{1}^{+}\right)$, each is a $\mathbf{P}_{v_{\alpha}}$-name for some countable $v_{\alpha} \subseteq \mu \backslash \lambda_{1}$. By the $\widetilde{\Delta}$-system lemma we can assume that $\alpha \neq \beta \Rightarrow v_{\alpha} \cap v_{\beta}=v^{*}$. Working in $\mathbf{V}^{\mathbf{P}_{\lambda_{1} \cup v^{*}}}$ we see that $\mathbf{P}_{v_{\alpha} \backslash v^{*}}$ is really the

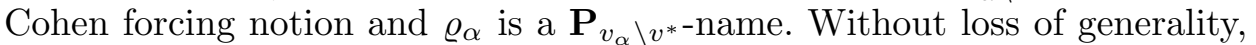
$v^{*}=\left[\lambda_{1}, \lambda_{1}+\omega\right), v_{\alpha}=v^{*} \cup\left\{\lambda_{1}+\omega+\alpha\right\}$ and all names $\varrho_{\alpha}$ are the same (under the natural isomorphism). So we have found a Cohen forcing name $\tau \in \mathbf{V}^{\mathbf{P}_{\lambda_{1}+\omega}}$ such that

$$
\begin{aligned}
& \text { if } c_{0}, c_{1} \text { are (mutually) Cohen reals over } \mathbf{V}^{\mathbf{P}_{\lambda_{1}+\omega}} \\
& \text { then } \mathbf{V}^{\mathbf{P}_{\lambda_{1}}}\left[c_{0}, c_{1}\right] \models\left(\tau^{c_{0}}, \mathcal{\tau}^{c_{1}}\right) \in \underset{\sim}{B} \& \underset{\sim}{\tau^{c_{0}}} \neq \mathcal{\tau}^{c_{1}} .
\end{aligned}
$$

But the Cohen forcing adds a perfect set of (mutually) Cohen reals. By absoluteness this produces a perfect set (in $\mathbf{V}^{\mathbf{P}_{\lambda_{1}}}$ ) whose square is contained in $\underset{\sim}{B}$. Once again by absoluteness we conclude that $\underset{\sim}{B}$ contains a perfect square in $\mathbf{V}^{\mathbf{P}_{\lambda}}$ already, a contradiction. $\mathbf{- 1}_{1.13}$

REMARK 1.14. Note that if $B$ is a subset of the plane $\left({ }^{\omega} \omega,{ }^{\omega} \omega\right)$ which is $G_{\delta}$ (i.e. $\bigcap_{n<\omega} U_{n}, U_{n}$ open, without loss of generality decreasing with $n$ ) and it contains an uncountable square $X \times X$ (so $X \subseteq{ }^{\omega} \omega$ is uncountable) then it contains a perfect square. Why? Let

$$
X^{\prime}=\left\{\eta \in X:(\forall n)\left(\exists^{\aleph_{1}} \nu\right)(\nu \in X \& \nu\lceil n=\eta\lceil n\} .\right.
$$

Let

$$
\begin{aligned}
& K=\left\{(u, n) \text { : for some } l \text { called } l(u, n), u \subseteq^{l} \omega\right. \text {, and } \\
& \eta, \nu \in u \& \eta \triangleleft \eta^{\prime} \in{ }^{\omega} \omega \& \nu \triangleleft \nu^{\prime} \in{ }^{\omega} \omega \Rightarrow\left(\eta^{\prime}, \nu^{\prime}\right) \in U_{n} \\
& \text { and } \left.\eta \in u \& \eta \triangleleft \eta^{\prime} \in{ }^{\omega} \omega \Rightarrow\left(\eta^{\prime}, \eta^{\prime}\right) \in U_{n}\right\} \text {, } \\
& K^{\prime}=\left\{(u, n) \in K \text { : for some } \bar{\nu}=\left\langle\nu_{\varrho}: \varrho \in u\right\rangle \text { we have } \nu_{\varrho} \in X^{\prime}, \varrho \triangleleft \nu_{\varrho}\right\} \text {. }
\end{aligned}
$$


Then

(a) $K^{\prime} \neq \emptyset$, in fact if $\eta_{1}, \ldots, \eta_{m} \in X^{\prime}$ are pairwise distinct and $n<\omega$, then $\left(\left\{\eta_{i} \mid l: i=1, \ldots, m\right\}, n\right) \in K$ for any $l$ large enough,

(b) if $(u, n) \in K^{\prime}$ as exemplified by $\bar{\nu}=\left\langle\nu_{\varrho}: \varrho \in u\right\rangle$ and $\varrho^{*} \in u$, $\nu^{\prime} \in X^{\prime} \backslash\left\{\nu_{\varrho^{*}}\right\}, \nu^{\prime} \uparrow l=\nu_{\varrho^{*}} \backslash l$ then for any $l^{\prime} \in(l, \omega)$ and $n^{\prime}>n$ large enough, we have $\left(\left\{\nu_{\varrho}\right\rceil l^{\prime}: \varrho \in u\right\} \cup\left\{\nu^{\prime}\left\lceil l^{\prime}\right\}, n^{\prime}\right) \in K^{\prime}$.

The following depends on $\S 3$ :

TheOREM 1.15. Assume $M A$ and $2^{\aleph_{0}} \geq \lambda_{\omega_{1}}\left(\aleph_{0}\right)$ or $2^{\aleph_{0}}>\mu$. Then: there is a Borel subset of the plane with a $\mu$-square but with no perfect square iff $\mu<\lambda_{\omega_{1}}\left(\aleph_{0}\right)$.

Pr o of. The first clause implies the second by 1.12. If the second clause holds, let $\mu \leq \lambda_{\alpha}\left(\aleph_{0}\right)$ and $\alpha<\omega_{1}$. By 3.2(6) letting $\eta_{i} \in \omega_{2}$ for $i<\mu$ be pairwise distinct we can find an $\omega$-sequence of $(2,2)$-trees $\bar{T}$ such that $\left(\eta_{i}, \eta_{j}\right) \in \bigcup_{n} \lim \left(T_{n}\right)$ for $i, j<\mu$ and $\operatorname{degsq}(\bar{T})=\alpha$ (just use $A=\left\{\left(\eta_{i}, \eta_{j}\right)\right.$ : $i, j<\mu\}$ there). By 3.2(3) the set $\bigcup_{n} \lim \left(T_{n}\right)$ contains no $\lambda_{\alpha+1}\left(\aleph_{0}\right)$-square.

FACT 1.16. Assume $\mathbf{P}$ is adding $\mu>\kappa$ Cohen reals or random reals and $\kappa>2^{\aleph_{0}}$. Then in $\mathbf{V}^{\mathbf{P}}$ we have

$(*)_{\kappa} \quad$ there is no Borel set (or analytic) $B \subseteq{ }^{\omega} 2 \times{ }^{\omega} 2$ such that

(a) there are $\eta_{\alpha} \in \omega^{\omega}$ for $\alpha<\kappa$ such that $\left[\alpha \neq \beta \Rightarrow \eta_{\alpha} \neq \eta_{\beta}\right]$ and $\left(\eta_{\alpha}, \eta_{\beta}\right) \in B$ for $\alpha, \beta<\kappa$,

(b) B contains no perfect square.

P r o of. Straightforward as in the (last) stage $\mathrm{G}$ of the proof of Theorem 1.13 (except that no relevance of (7) of stage A there).

Let $\mathbf{P}$ be adding $\left\langle\underline{r}_{\alpha}: \alpha<\mu\right\rangle$, and assume $p \in \mathbf{P}$ forces that a Borel set $\underset{\sim}{B}$ and $\left\langle\eta_{\alpha}: \alpha<\kappa\right\rangle$ are as in clauses (a), (b) above. Let $\eta_{\alpha}$ be names in $\mathbf{P}_{v_{\alpha}}=\mathbf{P}\left\lceil\left\{\widetilde{\widetilde{r}}_{\beta}: \beta \in v_{\alpha}\right\}\right.$, and $\underset{\sim}{B}$ be a name in $\mathbf{P}_{v}=\mathbf{P}\left\lceil\left\{\underline{\sim}_{\beta}: \widetilde{\beta} \in v\right\}\right.$ where $v$, $v_{\beta}$ are countable subsets of $\kappa$. Without loss of generality, $\left\langle v_{\alpha}: \alpha<\left(2^{\aleph_{0}}\right)^{+}\right\rangle$ is a $\Delta$-system with heart $v$ and $\operatorname{otp}\left(v_{\alpha} \backslash v\right)=\operatorname{otp}\left(v_{0} \backslash v\right)$. In $\mathbf{V}^{\mathbf{P}_{v}}$ we have $\underset{\sim}{B}$ and $2^{\aleph_{0}}=\left(2^{\aleph_{0}}\right) \mathbf{V}$, so we can assume $v=\emptyset$ and $\operatorname{otp}\left(v_{\alpha}\right)$ does not depend on $\alpha$.

Without loss of generality, the order preserving function $f_{\alpha, \beta}$ from $v_{\alpha}$ onto $v_{\beta}$ maps $\eta_{\alpha}$ to $\eta_{\beta}$. So for $Q=$ Cohen in the Cohen case we have a name $\mathcal{\tau}$ such that $\Vdash_{\text {Cohen }}^{\widetilde{\tau}} \underset{\sim}{\tau}(\underset{\sim}{r}) \in{ }^{\omega} 2$ is new", $\Vdash_{\text {Cohen } \times \text { Cohen }}$ " $\left(\underset{\sim}{\mathcal{\tau}}\left(\underset{\sim}{r_{1}}\right) ; \mathcal{\sim}\left(\underset{\sim}{r_{2}}\right)\right) \in B$ ", and we can finish easily. The random case is similar. 1.16

CONCLUSION 1.17. (1) For $\kappa \in\left(\aleph_{1}, \aleph_{\omega_{1}}\right)$ the statement $(*)_{\kappa}$ of 1.16 is not decided by $Z F C+2^{\aleph_{0}}>\aleph_{\omega_{1}}$ (i.e. it and its negation are consistent with $Z F C)$.

(2) 1.16 applies to the forcing notion of 1.13 (with $\mu$ instead of $2^{\aleph_{0}}$ ). 
Proof. (1) Starting with a universe V satisfying CH, Fact 1.16 shows the consistency of "yes". As by 1.7(1) we know that $\lambda_{\omega_{1}}\left(\aleph_{0}\right) \geq \aleph_{\omega_{1}}$ and $\aleph_{\omega_{1}}>\kappa$ (by assumption), Theorem 1.15 (with the classical consistency of MA $+2^{\aleph_{0}}>\aleph_{\omega_{1}}$ ) gives the consistency of "no" (in fact, in both cases it works for all $\kappa$ simultaneously).

(2) Left to the reader.

2. Some model-theoretic related problems. We turn to the modeltheoretic aspect: getting Hanf numbers below the continuum, i.e. if $\psi \in$ $L_{\omega_{1}, \omega}$ has a model of cardinality $\geq \lambda_{\omega_{1}}\left(\aleph_{0}\right)$ then it has a model of cardinality continuum. We show that $\operatorname{Pr}_{\omega_{1}}(\lambda)$ is equivalent to a statement of the form "if $\psi \in L_{\omega_{1}, \omega}$ has a model of cardinality $\lambda$ then it has a model generated by an "indiscernible" set indexed by "2" (the indiscernibility is with respect to the tree $\left(\omega \geq 2, \triangleleft, \cap,<_{\mathrm{lx}},<_{\mathrm{lg}}\right)$, where $\triangleleft$ is being initial segment, $\eta \cap \nu=$ maximal $\varrho$ with $\varrho \unlhd \eta \& \varrho \unlhd \nu,<_{\text {lx }}$ is lexicographic order, $\eta<_{\text {lx }} \nu$ iff $\left.\lg (\eta)<\lg (\nu)\right)$. This gives sufficient conditions for having many nonisomorphic models and also gives an alternative proof of 1.12 .

We also deal with the generalization to $\bar{\lambda}$-models, i.e. fixing the cardinalities of several unary predicates (and point to $\lambda$-like models).

Claim 2.1. The following are equivalent for a cardinal $\lambda$ :

(1) $\operatorname{Pr}_{\omega_{1}}(\lambda)$.

(2) If $\psi \in L_{\omega_{1}, \omega}$ has a model $M$ with $\left|R^{M}\right| \geq \lambda$ ( $R$ is a unary predicate) then $\psi$ has a model of cardinality continuum; moreover, for some countable first order theory $\mathbf{T}_{1}$ with Skolem functions such that $\tau(\psi) \subseteq \tau\left(\mathbf{T}_{1}\right)$ and a model $M_{1}$ of $\mathbf{T}_{1}$ and $a_{\eta} \in R^{M_{1}}$ for $\eta \in{ }^{\omega} 2$ we have:

$(*)_{0} M_{1} \models \psi$,

$(*)_{1} M_{1}, a_{\eta}\left(\eta \in{ }^{\omega} 2\right)$ are as in [Sh a, VII, $\left.\S 4\right]=[$ Sh c, VII, §4], i.e.:

(a) $M_{1}$ is the Skolem hull of $\left\{a_{\eta}: \eta \in{ }^{\omega} 2\right\}$ and $\eta \neq \nu \Rightarrow a_{\eta} \neq a_{\nu}$,

(b) for every $n<\omega$ and a first order formula $\varphi=\varphi\left(x_{0}, \ldots, x_{n-1}\right)$ $\in L\left(\mathbf{T}_{1}\right)$ there is $n^{*}<\omega$ such that for every $k \in\left(n^{*}, \omega\right)$, $\eta_{0}, \ldots, \eta_{n-1} \in{ }^{\omega} 2$ and $\nu_{0}, \ldots, \nu_{n-1} \in{ }^{\omega} 2$ satisfying

$$
\bigwedge_{m<n} \eta_{m}\left\lceil k=\nu_{m}\left\lceilk \quad \text { and } \quad \bigwedge _ { m < l < n } \eta _ { m } \left\lceil k \neq \eta_{l}\lceil k\right.\right.\right.
$$

we have

$$
M_{1}=" \varphi\left[a_{\eta_{0}}, \ldots, a_{\eta_{n-1}}\right] \equiv \varphi\left[a_{\nu_{0}}, \ldots, a_{\nu_{n-1}}\right] " .
$$

Note that necessarily $a_{\eta} \notin$ Skolem hull $M_{M_{1}}\left\{a_{\nu}: \nu \in{ }^{\omega} 2 \backslash\{\eta\}\right\}$.

(c) $a_{\eta} \in R^{M_{1}}$. 
REMARK 2.2. In (2) we can replace the assumption by "If $\psi \in L_{\omega_{1}, \omega}$ has, for every $\lambda^{\prime}<\lambda$, a model $M$ with $\left|R^{M}\right| \geq \lambda^{\prime}$ then ..." (and still the new version of $(2)$ is equivalent to (1)).

Pro of (of Claim 2.1). (1) $\Rightarrow(2)$. Just as in [Sh 37] + [Sh 49]: we can assume $\|M\|=\lambda$ and moreover $|M|=\lambda$. Let $M_{1}$ be an expansion of $M$ by names for subformulas of $\psi$, a pairing function, and then by Skolem functions. Let $\mathbf{T}_{1}$ be the first order theory of $M_{1}$. There is (see [Ke71]) a set $\Gamma$ of countably many types $p(x)$ such that $M_{1}$ omits every $p(x) \in \Gamma$ and if $M_{1}^{\prime}$ is a model of $\mathbf{T}_{1}$ omitting every $p(x) \in \Gamma$ then $M_{1}^{\prime}$ is a model of $\psi$ (just for each subformula $\bigwedge_{n<\omega} \psi_{n}(\bar{x})$ of $\psi$, we have to omit a type; we can use 1-types as we have a pairing function). Let us define

$Y=\left\{v \subseteq{ }^{\omega>} 2: v\right.$ is finite nonempty, its members are pairwise $\triangleleft$-incomparable and $v \subseteq{ }^{n} 2 \cup^{n+1} 2$ for some $\left.n\right\}$, $Z=\left\{\left(v, \varphi\left(\ldots, x_{\eta}, \ldots\right)_{\eta \in v}\right): v \in Y, \varphi\right.$ a formula in $T_{1}$ with the set of free variables included in $\left\{x_{\eta}: \eta \in v\right\}$ and for every $\alpha<\omega_{1}$ there are $a_{\eta}^{\alpha} \in R^{M}$ for $\eta \in v$ such that: $\left[\eta \neq \nu\right.$ from $\left.v \Rightarrow a_{\eta}^{\alpha} \neq a_{\nu}^{\alpha}\right], \operatorname{rk}^{0}\left(\left\{a_{\eta}^{\alpha}\right.\right.$ : $\eta \in v\}, M) \geq \alpha$ and $\left.M \models \varphi\left[\ldots, a_{\eta}^{\alpha}, \ldots\right]_{\eta \in v}\right\}$.

We say for $\left(v_{l}, \varphi_{l}\right) \in Z(l=1,2)$ that $\left(v_{2}, \varphi_{2}\right) \in \operatorname{succ}\left(v_{1}, \varphi_{1}\right)$ if for some $\eta \in v_{1}$ (called $\left.\eta\left(v_{1}, v_{2}\right)\right)$ we have $v_{2}=\left(v_{1} \backslash\{\eta\}\right) \cup\left\{\eta^{\wedge}\langle 0\rangle, \eta^{\wedge}\langle 1\rangle\right\}$. For $i<2$ define the function $h_{i}: v_{1} \rightarrow v_{2}$ by $h_{i}(\nu)$ is $\nu$ if $\nu \neq \eta$ and $h_{i}(\nu)=\eta^{\wedge}\langle i\rangle$ if $\nu=\eta$. We demand, for $i=0,1$,

$$
\varphi_{2} \vdash \varphi_{1}\left(\ldots, x_{h_{i}(\nu)}, \ldots\right)_{\nu \in v_{1}} .
$$

Choose inductively $\left\langle\left(v_{l}, \varphi_{l}\right): l<\omega\right\rangle$ such that $\left(v_{l+1}, \varphi_{l+1}\right) \in \operatorname{succ}\left(v_{l}, \varphi_{l}\right)$ is generic enough, i.e.

$(\otimes)_{1} \quad$ if $\varphi=\varphi\left(x_{0}, \ldots, x_{k-1}\right) \in L\left(\mathbf{T}_{1}\right)$ then for some $l<\omega$ and all $m \in$ $[l, \omega)$ and $\eta_{0}, \ldots, \eta_{k-1} \in v_{m}$ we have either

$$
\varphi_{m} \vdash \varphi\left(x_{\eta_{0}}, \ldots, x_{\eta_{k-1}}\right) \quad \text { or } \quad \varphi_{m} \vdash \neg \varphi\left(x_{\eta_{0}}, \ldots, x_{\eta_{k-1}}\right),
$$

$(\otimes)_{2} \quad$ for every $p(x) \in \Gamma$ and every function symbol $f=f\left(x_{0}, \ldots, x_{n-1}\right)$ (note: in $\mathbf{T}_{1}$ a definable function is equivalent to some function symbol), for some $l<\omega$, all $m \in[l, \omega)$ and all $\eta_{0}, \ldots, \eta_{n_{1}} \in v_{m}$ there is $\psi(x) \in p(x)$ such that

$$
\varphi_{m} \vdash \neg \psi\left(f\left(x_{\eta_{0}}\right), \ldots, f\left(x_{\eta_{n-1}}\right)\right) .
$$

It is straightforward to carry out the induction (to simplify you may demand in $(\otimes)_{1},(\otimes)_{2}$ just "for arbitrarily large $m \in[l, \omega)$ ", this does not matter and the stronger version of $(\otimes)_{1},(\otimes)_{2}$ can be obtained (replacing the ${ }^{\omega>} 2$ by a perfect subtree $T$ and then renaming $a_{\eta}$ for $\eta \in \lim (T)$ as $a_{\eta}$ for $\left.\eta \in{ }^{\omega} 2\right)$ ). Then define the model by compactness. 
$(2) \Rightarrow(1)$. If not, then $\operatorname{NPr}_{\omega_{1}}(\lambda)$, hence for some model $M$ with vocabulary $\tau$ having $|\tau| \leq \aleph_{0}$ and of cardinality $\lambda$ we have $\alpha(*):=\operatorname{rk}^{0}(M)<\omega_{1}$. Let $\psi_{\alpha(*)} \in L_{\omega_{1}, \omega}(\tau)$ be as in 2.3 below, so necessarily $M \models \psi_{\alpha(*)}$. Apply to it clause (2) which holds by our present assumption (with $R^{M}=\lambda$ ), so $\psi_{\alpha(*)}$ has a model $M_{1}$ as there (so $\left.M_{1}=\psi_{\alpha(*)}\right)$. But $\left\{a_{\eta}: \eta \in{ }^{\omega} 2\right\}$ easily witnesses $\operatorname{rk}^{0}\left(M_{1}\right)=\infty$, and moreover, for every nonempty finite $w \subseteq\left\{a_{\eta}: \eta \in{ }^{\omega} 2\right\}$ and an ordinal $\alpha$ we have $\operatorname{rk}^{0}(w, M) \geq \alpha$. This can be easily proved by induction on $\alpha$ (using $(*)_{1}$ (b) of $(2)$ (and $\eta \neq \nu \in \omega_{2} \Rightarrow a_{\eta} \neq a_{\nu}$ of $\left.(*)_{1}(\mathrm{a})\right)$ ). $\mathbf{-}_{2.1}$

FACT 2.3. (1) For every $\alpha<\kappa^{+}$and vocabulary $\tau$ with $|\tau| \leq \kappa$ there is a sentence $\psi_{\alpha} \in L_{\kappa^{+}, \omega}[\tau]$ (of quantifier depth $\alpha$ ) such that for any $\tau$-model $M$,

$$
M \models \psi_{\alpha} \quad \text { iff } \quad \operatorname{rk}^{0}\left(M ;<\aleph_{0}\right)=\alpha .
$$

(2) For every $\alpha<\theta^{+}, l \in\{0,1\}$ and vocabulary $\tau$ with $|\tau| \leq \theta$ there is a sentence $\psi \in L_{\theta^{+}, \omega}(\exists \geq \kappa)[\tau]\left(\exists^{\geq \kappa}\right.$ is the quantifier "there are $\geq \kappa$ many") such that for any $\tau$-model $M: M \models \psi_{\alpha}^{l}$ iff $\operatorname{rk}^{l}(M ;<\kappa, \theta)=\alpha$.

Pr o of. Easy to check. $\mathbf{m}_{2.3}$

Hence (just as in [Sh a, VIII, 1.8(2)]):

Conclusion 2.4. Assume $\tau$ is a countable vocabulary. If $\psi \in L_{\omega_{1}, \omega}(\tau)$, $R$ is a unary predicate, $\tau_{0} \subseteq \tau, \Delta \subseteq\left\{\varphi(x): \varphi \in L_{\omega_{1}, \omega}\left(\tau_{0}\right)\right\}$ is countable and for some transitive model $\mathbf{V}_{1}$ of ZFC (maybe a generic extension of $\mathbf{V}$ or an inner model as long as $\psi, \Delta \in \mathbf{V}_{1}$ and $\mathbf{V}_{1}=" \psi \in L_{\omega_{1}, \omega}(\tau), \Delta \subseteq\{\varphi(x)$ : $\left.\left.\varphi \in L_{\omega_{1}, \omega}\left(\tau_{0}\right)\right\} "\right)$ we have

$$
\begin{aligned}
& \mathbf{V}_{1} \models \operatorname{~} \operatorname{Pr}_{\omega_{1}}(\lambda), \text { and } \psi \text { has a model } M \text { with } \\
& \quad \lambda \leq\left|\left\{\{\varphi(x): M \models \varphi[a], \varphi(x) \in \Delta\}: a \in R^{M}\right\}\right| " .
\end{aligned}
$$

Then:

(1) We can find a model $N$ of $\psi$ with Skolem functions and $a_{\alpha} \in R^{N}$ for $\alpha<2^{\aleph_{0}}$ such that for each $\alpha<2^{\aleph_{0}}$ the type $p_{\alpha}=\{ \pm \varphi(x): N \models$ $\pm \varphi\left[a_{\alpha}\right]$ and $\left.\varphi(x) \in \Delta\right\}$ is not realized in the Skolem hull of

$$
\left\{a_{\beta}: \beta<2^{\aleph_{0}} \text { and } \beta \neq \alpha\right\} .
$$

(2) $\operatorname{So}|\{M / \approx: M=\psi,\|M\|=\lambda\}| \geq \min \left\{2^{\lambda}, \beth_{2}\right\} ;$ really $\left\{\left(M\left\lceil\tau_{0}\right) / \approx\right.\right.$ : $M \models \psi$ and $M$ has cardinality $\lambda\}$ has cardinality $\geq \min \left\{2^{\lambda}, \beth_{2}\right\}$. Moreover, we can find such a family of models no one of them embeddable into another by an embedding preserving $\pm \varphi(x)$ for $\varphi \in \Delta$. $\mathbf{m}_{2.4}$

A natural generalization of 2.1 is

CLAIM 2.5. (1) For cardinals $\lambda>\kappa \geq \aleph_{0}$ the following are equivalent:

(a) $\operatorname{Pr}_{\kappa^{+}}(\lambda ; \kappa)$. 
(b) If $M$ is a model, $\tau(M)$ countable, $R, R_{0} \in \tau(M)$ unary predicates, $\left|R_{0}^{M}\right| \leq \kappa, \lambda \leq\left|R^{M}\right|$ then we can find $M_{0}, M_{1}, a_{\eta}\left(\eta \in{ }^{\omega} 2\right)$ such that:

(i) $M_{1}$ is a model of the (first order) universal theory of $M$ (and is a $\tau(M)$-model ,

(ii) $a_{\eta} \in R^{M_{1}}$ for $\eta \in{ }^{\omega} 2$ are pairwise distinct,

(iii) $M_{1}$ is the closure of $\left\{a_{\eta}: \eta \in{ }^{\omega} 2\right\} \cup M_{0}$ under the functions of $M_{1}$, so

( $\alpha) M_{1}$ also includes the individual constants of $M$; in general $\left\|M_{1}\right\|=2^{\aleph_{0}}$

$(\beta)$ if $\tau(M)$ has predicates only then $\left|M_{1}\right|=\left\{a_{\eta}: \eta \in{ }^{\omega} 2\right\} \cup$ $\left|M_{0}\right|$,

(iv) $M_{0}$ is countable, $M_{0} \subseteq M, M_{0} \subseteq M_{1}, M_{0}=\operatorname{cl}_{M}\left(M_{0} \cap\right.$ $\left.R_{0}^{M}\right), R_{0}^{M_{1}}=R_{0}^{M_{0}}\left(\subseteq R_{0}^{M}\right)$; in fact, we can have:

$(*)\left(M_{1}, c\right)_{c \in M_{0}}$ is a model of the universal theory of $(M, c)_{c \in M_{0}}$,

(v) for every $n<\omega$ and a quantifier free first order formula $\varphi=$ $\varphi\left(x_{0}, \ldots, x_{n-1}\right) \in L(\tau(M))$ there is $n^{*}<\omega$ such that for every $k \in\left(n^{*}, \omega\right)$ and $\eta_{0}, \ldots, \eta_{n-1} \in{ }^{\omega} 2, \nu_{0}, \ldots, \nu_{n-1} \in{ }^{\omega} 2$ satisfying

$$
\bigwedge_{m<n} \eta_{m}\left\lceil k=\nu_{m}\left\lceilk \quad \text { and } \quad \bigwedge _ { m < l < n } \eta _ { m } \left\lceil k \neq \eta_{l} \uparrow k\right.\right.\right.
$$

we have

$$
M_{1}=" \varphi\left[a_{\eta_{0}}, \ldots, a_{\eta_{n-1}}\right] \equiv \varphi\left[a_{\nu_{0}}, \ldots, a_{\nu_{n-1}}\right] " ;
$$

we can even allow parameters from $M_{0}$ in $\varphi$ (but $k$ depends on them).

(2) For cardinals $\lambda>\kappa \geq \aleph_{0}$ the following are equivalent:

(a) $\operatorname{Pr}_{\omega_{1}}(\lambda ; \kappa)$.

(b)' Like (b) above, but we omit " $M_{0} \subseteq M$ ".

Remark 2.6. (1) See 4.6, 4.7 how to use Claim 2.5.

(2) In (b), if $M$ has Skolem functions then we automatically get also:

(i) ${ }^{+} M_{1}$ is a model of the first order theory of $M$,

(iii) ${ }^{+} M_{1}$ is the Skolem hull of $\left\{a_{\eta}: \eta \in{ }^{\omega} 2\right\} \cup M_{0}$,

(iv) ${ }^{+} M_{0} \prec M, M_{0} \prec M_{1}, M_{0}$ countable (and $R_{0}^{M_{1}}=R_{0}^{M_{0}} \subseteq R_{0}^{M}$ ),

$(\mathrm{v})^{+}$clause (v) above holds even for $\varphi$ any (first order) formula of $L_{\omega, \omega}(\tau(M))$. 
Proof. (1)(a) $\Rightarrow$ (b). Like the proof of $2.1(1) \Rightarrow(2)$, applied to $(M, c)_{c \in R_{0}^{M}}$, but the set $M_{0} \cap R_{0}^{M}$ is chosen by finite approximation, i.e. (letting $Y$ be as there and $\tau=\tau(M)$ ) we let

$Z=\left\{\left(v, \varphi\left(\ldots, x_{\eta}, \ldots\right)_{\eta \in v}, A\right): v \in Y, \varphi\right.$ a quantifier free formula

in $L_{\omega, \omega}(\tau)$ with set of free variables included in $\left\{x_{\eta}: \eta \in v\right\}$

and parameters from $A, A$ is a finite subset of $R_{0}^{M}$,

and for every ordinal $\alpha<\kappa^{+}$there are $a_{\eta}^{\alpha} \in R^{M}$ for $\eta \in v$

such that $\left[\eta \neq \nu\right.$ from $\left.v \Rightarrow a_{\eta}^{\alpha} \neq a_{\nu}^{\alpha}\right]$ and

$\operatorname{rk}\left(\left\{a_{\eta}^{\alpha}: \eta \in v\right\}, M\right) \geq \alpha$ and $\left.M \models \varphi\left[\ldots, a_{\eta}^{\alpha}, \ldots\right]_{\eta \in v}\right\}$.

We need the "for every $\alpha<\kappa^{+}$" here because we want to fix elements of $R_{0}^{M}$, and there are $\kappa$ possible choices.

$\neg(\mathrm{a}) \Rightarrow \neg$ (b). Like the proof of 2.3 ; assume $\operatorname{NPr}_{\alpha}(\lambda ; \kappa), \alpha<\kappa^{+}$, let $M$ witness it, choose $R_{0}=\alpha+1, R=\lambda$; without loss of generality, $\tau(M)=\left\{R_{n, \zeta}: n<\omega, \zeta<\kappa\right\}, R_{n, \zeta}$ is $n$-place, in $M$ every quantifier free formula is equivalent to some $R_{n, \zeta}$. Let $R_{n, k}^{*}:=\left\{\left(i_{0}, \ldots, i_{n-1}, \beta, \zeta\right): M \models\right.$ $R_{n, \zeta}\left(i_{0}, \ldots, i_{n-1}\right),\left\{i_{0}, \ldots, i_{n-1}\right\}$ is with no repetition, increasing for simplicity, and $\operatorname{rk}\left(\left\{i_{0}, \ldots, i_{n-1}\right\}, M ; \kappa\right)=\beta$, with $\operatorname{rk}\left(\left\{i_{0}, \ldots, i_{n-1}\right\}, M ; \kappa\right) \nsupseteq \beta+1$ being witnessed by $\left.\varphi\left(\left\{i_{0}, \ldots, i_{n-1}\right\}\right)=R_{n, \zeta}, k\left(\left\{i_{0}, \ldots, i_{n-1}\right\}\right)=k\right\}$ where the functions $\varphi, k$ are as in the proof of 1.13. Let $M$ be $\left(\lambda,<, R, R_{0}\right.$, $\left.R_{n, k}^{*}\right)_{n \in(0, \omega), k<n}$ expanded by Skolem functions.

So assume toward a contradiction that (b) holds, hence for this model $M$ there are models $M_{0}, M_{1}$ and $a_{\eta} \in M_{1}$ for $\eta \in{ }^{\omega} 2$ as required in clauses (i)-(v) of (b) of claim 2.5. Choose a nonempty finite subset $w$ of ${ }^{\omega} 2$ and $\beta$ and $\zeta$ such that letting $w=\left\{\eta_{0}, \ldots, \eta_{m-1}\right\}$ with $a_{\eta_{l}}<{ }^{M_{1}} a_{\eta_{l+1}}$, we have

( $\alpha) M_{1} \models R_{m, k}^{*}\left(a_{\eta_{0}}, \ldots, a_{\eta_{m-1}}, \beta, \zeta\right)$,

( $\beta) \beta \in R_{0}^{M_{0}}(\subseteq \alpha)$,

( $\gamma) \beta$ minimal under those constraints.

Note that there are $m, \eta_{0}, \ldots, \eta_{m-1}, \beta$ and $\zeta$ such that $(\alpha)$ holds: for every nonempty $w \subseteq{ }^{\omega} 2$, as $M_{1}$ is elementarily equivalent to $M$ there are $\beta, \zeta$ as required in $(\alpha)$. Now $(\alpha)$ implies $\zeta \in R_{0}^{M_{1}}$, but $R_{0}^{M_{1}}=R_{0}^{M_{0}}$, so clause $(\beta)$ holds too, and so we can satisfy $(\gamma)$ too as the ordinals are well ordered. Let $\varphi^{\prime}=\varphi\left(x_{0}, \ldots, x_{m-1}, \beta, \zeta\right)$; note that the parameters are from $R_{0}^{M_{1}}$ (as $M_{1}$ is elementarily equivalent to $M$ ) and hence from $R_{0}^{M_{0}} \subseteq M$, and clause (v) (of (b) of 2.5) applies to $\varphi^{\prime}$ and $\left\langle\eta_{0}, \ldots, \eta_{m-1}\right\rangle$, giving $n^{*}<\omega$. We can find $\eta_{k}^{\prime} \in$ $\omega_{2}, \eta_{k}^{\prime} \neq \eta_{k}, \eta_{k}^{\prime}\left\lceil n^{*}=\eta_{k}\left\lceil n^{*}\right.\right.$, and easily for $w^{\prime}=\left\{\eta, \ldots, \eta_{m-1}, \eta_{k}^{\prime}\right\}$ we can find $\beta^{\prime}<\beta, \zeta^{\prime}<\kappa$ and $k$ such that $M_{1} \models R_{m+1, k^{\prime}}\left(a_{\eta_{0}}, \ldots, a_{\eta_{m-1}}, a_{\eta_{k}^{\prime}}, \beta^{\prime}, \zeta^{\prime}\right)$; then if $\beta \geq 0$ we get a contradiction to clause $(\gamma)$ above. If $\beta=0$ we use clause (i) to copy the situation to $M$ and get a contradiction.

(2) Similar proof. 
Notation 2.7. Let $\bar{\lambda}$ denote a finite (or countable) sequence of pairs of infinite cardinals $\left\langle\left(\lambda_{\zeta} ; \kappa_{\zeta}\right): \zeta<\zeta(*)\right\rangle$ such that $\kappa_{\zeta}$ increases with $\zeta$, so e.g. $\bar{\lambda}^{\oplus}=\left\langle\left(\lambda_{\zeta}^{\oplus}, \kappa_{\zeta}^{\oplus}\right): \zeta<\zeta^{\oplus}(*)\right\rangle$. We shall identify a strictly increasing $\bar{\kappa}=\left\langle\kappa_{\zeta}: \zeta \leq \zeta(*)\right\rangle$ with $\left\langle\left(\kappa_{\zeta+1} ; \kappa_{\zeta}\right): \zeta<\zeta(*)\right\rangle$.

Let $R, R_{0}, Q_{0}, \ldots, R_{\zeta(*)-1}, Q_{\zeta(*)-1}$ be fixed unary predicates and $\bar{R}=$ $\left\langle\left(R_{\zeta}, Q_{\zeta}\right): \zeta<\zeta(*)\right\rangle$.

A $\bar{\lambda}$-model $M$ is a model $M$ such that $R, R_{\zeta}, Q_{\zeta} \in \tau(M)$ are all unary predicates, $\left|R_{\zeta}^{M}\right|=\lambda_{\zeta},\left|Q_{\zeta}^{M}\right|=\kappa_{\zeta}$ for $\zeta<\zeta(*), Q_{\zeta}^{M} \subseteq R_{\zeta}^{M}$ and $\left\langle R_{\zeta}^{M}: \zeta<\right.$ $\zeta(*)\rangle$ are pairwise disjoint, and $R^{M}=\bigcup_{\zeta<\zeta(*)} R_{\zeta}^{M}$.

For $a \in R^{M}$ let $\zeta(a)$ be the $\zeta$ such that $a \in R_{\zeta}^{M}$ (e.g. $R_{\zeta}^{M}=\lambda_{\zeta} \backslash \bigcup_{\xi<\zeta} \lambda_{\xi}$, $\left.\kappa_{\zeta+1}=\lambda_{\zeta}\right)$. For a $\bar{\lambda}$-model $M$ we say that $a \in \operatorname{cl}_{\kappa}(A, M)$ if $A \cup\{a\} \subseteq$ $M$ and for some $n<\omega$, and quantifier free formula $\varphi\left(x_{1}, y_{1}, \ldots, y_{n}\right)$ and $b_{1}, \ldots, b_{n} \in A$ we have

$$
M \models \varphi\left(a, b_{1}, \ldots, b_{n}\right) \&\left(\exists^{\leq \kappa} x\right) \varphi\left(x, b_{1}, \ldots, b_{n}\right) .
$$

Definition 2.8. (1) For $l<6, \bar{\lambda}$ as in Notation 2.7, and an ordinal $\alpha$ let $\operatorname{Pr}_{\alpha}^{l}(\bar{\lambda} ; \theta)$ mean that for every $\bar{\lambda}$-model $M$ with $|\tau(M)| \leq \theta$ we have $\operatorname{rk}^{l}(M, \bar{\lambda}) \geq \alpha$ (and $\operatorname{NPr}_{\alpha}^{l}(\bar{\lambda}, \theta)$ is the negation; if $\theta$ is omitted it means $\kappa_{0}$; remember $\left.\bar{\lambda}=\left\langle\left(\lambda_{\zeta}, \kappa_{\zeta}\right): \zeta<\zeta(*)\right\rangle\right)$ where the rank is defined in part (2) below.

(2) For a $\bar{\lambda}$-model $M, \operatorname{rk}^{l}(M, \bar{\lambda})=\sup \left\{\operatorname{rk}^{l}(w, M, \bar{\lambda})+1: w \in\left[\bar{R}^{M}\right]^{*}\right\}$ where the rank is defined in part (3) below and:

$(\alpha)$ if $\zeta(*)$ is finite, then $\left[\bar{R}^{M}\right]^{*}=\left\{w: w\right.$ a finite subset of $R^{M}$ not disjoint from any $\left.R_{\zeta}^{M}\right\}$,

$(\beta)$ if $\zeta(*)$ is infinite, then

$$
\left[\bar{R}^{M}\right]^{*}=\left\{w: w \text { a finite nonempty subset of } R^{M}\right\} .
$$

(3) For a $\bar{\lambda}$-model $M$ and $w \in\left[R^{M}\right]^{*}$ we define the truth value of "rk ${ }^{l}(w, M ; \bar{\lambda}) \geq \alpha$ " by induction on $\alpha$ :

- CASE A, $\alpha=0$ :

$\operatorname{rk}^{l}(w, M ; \bar{\lambda}) \geq \alpha \quad$ iff $\quad$ no $a \in w \cap R^{M}$ belongs to $\operatorname{cl}_{\kappa_{\zeta(a)}}(w \backslash\{a\}, M)$.

- CAse B, $\alpha$ is a limit ordinal: $\operatorname{rk}^{l}(w, M ; \bar{\lambda}) \geq \alpha \quad$ iff $\operatorname{rk}^{l}(w, M ; \bar{\lambda}) \geq \beta$ for every ordinal $\beta<\alpha$.

- CASE C, $\alpha=\beta+1$. We demand two conditions:

$(\alpha)$ exactly as in Definition $1.1(3)(*)_{3}$ except that if $l=2,3,4,5$ we use $\kappa=\kappa_{\zeta\left(a_{k}\right)}^{+}$,

$(\beta)$ if $\zeta<\zeta(*)$ and $w \cap R_{\zeta}^{M}=\emptyset$ then $\operatorname{rk}^{l}(w \cup\{a\}, M ; \theta) \geq \beta$ for some $a \in R_{\zeta}^{M}$. 
Claim 2.9. The parallel of the following holds: 1.2 (+ statements in 1.1), also 1.3 (use $\alpha<\kappa_{0}^{+}$), 1.5(2) (for $\alpha \leq \kappa_{0}^{+}$), 1.10 (satisfying $\kappa_{0}^{+}$-c.c.) and we adopt 1.6.

Claim 2.10. If $\alpha$ is a limit ordinal and $\lambda_{\xi} \geq \beth_{\alpha}\left(\kappa_{\xi}\right)$ for every $\xi<\xi(*)$ then $\operatorname{Pr}_{\alpha}(\bar{\lambda})$.

Proof. Use indiscernibility and Erdős-Rado as in the proof of 1.8(1).

In more details, the induction hypothesis on $\alpha$ is, assuming $\zeta(*)<\omega$ : if $A \subseteq R^{M}$ and $\bigwedge_{\zeta<\zeta(*)}\left|A \cap R_{\zeta}^{M}\right| \geq \beth_{\omega \times \alpha}$ then for every $\beta<\alpha, k<\omega$ and every $\bar{m}=\left\langle m_{\zeta}: \zeta<\zeta(*)\right\rangle$ where $m_{\zeta} \in(0, \omega)$ for some $w \subseteq A$ we have $\bigwedge_{\zeta}\left|w \cap R_{\zeta}^{M}\right|=m_{\zeta}$ and $\operatorname{rk}(w ; M, \bar{\lambda}) \geq \omega \times \beta+k$. Then for $\alpha=\gamma+1$, choose distinct $a_{i}^{\zeta} \in A \cap R_{\zeta}^{M}\left(i<\beth_{\omega \times \gamma+m+m_{\zeta}}\right)$ and use polarized partition (see Erdős, Hajnal, Máté, Rado [EHMR]) on $\left\langle\left\langle a_{i}^{\zeta}: i<\beth_{\omega \times \alpha}\right\rangle: \zeta<\zeta(*)\right\rangle$. For $\zeta(*)$ infinite use $A \subseteq R^{M}$ such that $w_{A}=\left\{\zeta: A \cap R_{\zeta}^{M} \neq \emptyset\right\}$ is finite nonempty and $\zeta \in w_{A} \Rightarrow\left|A \cap R_{\zeta}^{M}\right| \geq \beth_{\omega \times \alpha}$, and proceed as above. $\mathbf{m}_{2.10}$

CLAIM 2.11. Let $\zeta(*)<\omega, \kappa_{0}^{\varepsilon}<\ldots<\kappa_{\zeta(*)}^{\varepsilon}$ and $\bar{\lambda}^{\varepsilon}=\left\langle\left(\kappa_{\xi+1}^{\varepsilon}, \kappa_{\xi}^{\varepsilon}\right): \xi<\right.$ $\zeta(*)\rangle($ for $\varepsilon \leq \omega)$.

(1) If $\operatorname{Pr}_{n}\left(\bar{\lambda}^{n}\right)$ for $n<\omega$, and for some $\theta \leq \kappa_{0}^{\omega}$ there is a tree $\mathcal{T} \in{ }^{\theta>}\left(\kappa_{0}^{\omega}\right)$ of cardinality $\leq \kappa_{0}^{\omega}$ with $\geq \kappa_{\zeta(*)}^{\omega} \theta$-branches, then:

$\otimes \quad$ every first order sentence which has a $\bar{\lambda}^{n}$-model for each $n$, also has a $\bar{\lambda}^{\omega}$-model,

$\otimes^{\prime} \quad$ if $\mathbf{T}$ is a first order theory of cardinality $\leq \kappa_{0}^{\omega}$ and every finite $\mathbf{T}^{\prime} \subseteq \mathbf{T}$ has a $\bar{\lambda}^{n}$-model for each $n$ then $\mathbf{T}$ has a $\bar{\lambda}^{\omega}$-model.

(2) So if $\bar{\lambda}^{\varepsilon}=\bar{\lambda}$ for $\varepsilon \leq \omega$ are as above then we have $\kappa_{0}^{\omega}$-compactness for the class of $\bar{\lambda}^{\omega}$-models, where

$(\oplus) \quad$ a class $\mathfrak{K}$ of models is $\kappa$-compact whenever for every set $\mathbf{T}$ of $\leq \kappa$ first order sentences, if every finite subset of $\mathbf{T}$ has a model in $\mathfrak{K}$ then $\mathbf{T}$ has a model in $\mathfrak{K}$.

(3) In part (1) we can use $\bar{\lambda}^{n}$ with domain $w_{n}$ if $w_{n} \subseteq w_{n+1}$ and $\zeta(*)=$ $\bigcup\left\{w_{n}: n<\omega\right\}$.

Proof. Straightforward if you have read [Sh 8], [Sh 18], [Sh 37] or will read the proof of 2.12 below (only that now the theory is not necessarily countable, no types omitted, and by compactness it is enough to deal with the case of $\zeta(*)$ finite). $\mathbf{m}_{2.11}$

Claim 2.12. Let $\zeta(*)<\omega_{1}$ and $\bar{\lambda}^{\varepsilon}=\left\langle\left(\kappa_{\xi+1}^{\varepsilon}, \kappa_{\xi}^{\varepsilon}\right): \xi<\zeta(*)\right\rangle$ for each $\varepsilon \leq \omega_{1}$ (and $\kappa_{\xi}^{\varepsilon}$ strictly increasing with $\xi$ ). If $\operatorname{Pr}_{\varepsilon}\left(\bar{\lambda}^{\varepsilon}\right)$ for every $\varepsilon<\omega_{1}$ and $\kappa_{\xi}^{\omega_{1}} \leq 2^{\aleph_{0}}$, and $\psi \in L_{\omega_{1}, \omega}$, and for each $\varepsilon<\omega_{1}$ there is a $\bar{\lambda}^{\varepsilon}$-model satisfying $\psi$, then there is a $\bar{\lambda}^{\omega_{1}}$-model satisfying $\psi$. 
Proof. For simplicity, again as in [Sh 8], [Sh 18], [Sh 37], let $M_{\varepsilon}$ be a $\bar{\lambda}^{\varepsilon}$-model of $\psi$ for $\varepsilon<\omega_{1}$. By expanding the $M_{\varepsilon}$ 's by a pairing function and giving names of subformulas of $\psi$ we have a countable first order theory $\mathbf{T}$ with Skolem functions, a countable set $\Gamma$ of 1-types and $M_{\varepsilon}^{+}$such that:

(a) $M_{\varepsilon}^{+}$is a $\bar{\lambda}^{\varepsilon}$-model of $\mathbf{T}$ omitting each $p \in \Gamma$,

(b) if $M$ is a model of $\mathbf{T}$ omitting every $p \in \Gamma$ then $M$ is a model of $\psi$.

Now as in the proof of 2.1 we can find a model $M^{+}$and $a_{\eta}^{\zeta}$ for $\zeta<\zeta(*)$ and $\eta \in{ }^{\omega} 2$ such that:

$(\alpha) M^{+}$is a model of $\mathbf{T}$.

( $\beta) a_{\eta}^{\zeta} \in R_{\zeta}^{M}$ and $\eta \neq \nu \Rightarrow a_{\eta}^{\zeta} \neq a_{\nu}^{\zeta}$.

$(\gamma)$ For every first order formula $\varphi\left(x_{0}, \ldots, x_{n-1}\right) \in L_{\omega, \omega}(\tau(\mathbf{T}))$ and ordinals $\zeta(0), \ldots, \zeta(n-1)<\zeta(*)$, there is $k<\omega$ such that if $\eta_{0}, \ldots, \eta_{n-1} \in{ }^{\omega} 2$, $\nu_{0}, \ldots, \nu_{n-1} \in{ }^{\omega} 2,\left\langle\eta_{l}\lceil k: l<n\rangle\right.$ is with no repetitions and $\eta_{l}\left\lceil k=\nu_{l}\lceil k\right.$ then $M^{+}=\varphi\left(a_{\eta_{0}}^{\zeta(0)}, \ldots, a_{\eta_{n-1}}^{\zeta(n-1)}\right) \equiv \varphi\left(a_{\nu_{0}}^{\zeta(0)}, \ldots, a_{\nu_{n-1}}^{\zeta(n-1)}\right)$.

( $\delta)$ If $\sigma\left(x_{0}, \ldots, x_{n-1}\right)$ is a term of $\tau(\mathbf{T})$ and $\zeta(0), \ldots, \zeta(n-1)<\zeta(*)$, and $p \in \Gamma$ then for some $k<\omega$ and any $\varrho_{0}, \ldots, \varrho_{n-1} \in{ }^{k} 2$ pairwise distinct there is $\varphi(x) \in p(x)$ such that

$$
\varrho_{l} \triangleleft \eta_{l} \in{ }^{\omega} 2 \Rightarrow M^{+} \models \neg \varphi\left(a_{\eta_{0}}^{\zeta(0)}, \ldots, a_{\eta_{n-1}}^{\zeta(n-1)}\right)
$$

(i.e. this is our way to omit the types in $\Gamma$ ).

$(\varepsilon)$ If $\zeta(0), \ldots, \zeta(n-1)<\zeta(*), \sigma\left(x_{0}, \ldots, x_{n-1}\right)$ is a term of $\tau(\mathbf{T})$, and $m<n$, then for some $k<\omega$, we have

(*) if $\eta_{0}, \ldots, \eta_{n-1} \in{ }^{\omega} 2, \nu_{0}, \ldots, \nu_{n-1} \in{ }^{\omega} 2, \eta_{l}\left\lceil k=\nu_{l}\left\lceil k,\left\langle\eta_{l}\lceil k: l<\omega\rangle\right.\right.\right.$ is without repetitions and $\zeta(l)<\zeta(m) \Rightarrow \eta_{l}=\nu_{l}$ then

$$
\begin{array}{r}
M^{+}=Q_{\zeta(m)}\left(\sigma\left(a_{\eta_{0}}^{\zeta(0)}, \ldots, a_{\eta_{n-1}}^{\zeta(n-1)}\right)\right) \& Q_{\zeta(m)}\left(\sigma\left(a_{\nu_{0}}^{\zeta(0)}, \ldots, a_{\nu_{n-1}}^{\zeta(n-1)}\right)\right) \\
\Rightarrow \sigma\left(a_{\eta_{0}}^{\zeta(0)}, \ldots, a_{\eta_{n-1}}^{\zeta(n-1)}\right)=\sigma\left(a_{\nu_{0}}^{\zeta(0)}, \ldots, a_{\nu_{n-1}}^{\zeta(n-1)}\right) .
\end{array}
$$

Now choose $Y_{\zeta} \subseteq{ }^{\omega} 2$ of cardinality $\lambda_{\zeta}^{\omega_{1}}$ and let $M^{*}$ be the $\tau(M)$-reduct of the Skolem hull in $M^{+}$of $\left\{a_{\eta}^{\zeta}: \zeta<\zeta(*)\right.$ and $\left.\eta \in Y_{\zeta}\right\}$. This is a model as required.

Conclusion 2.13. If $\mathbf{V}_{0} \models G C H, \mathbf{V}=\mathbf{V}_{0}^{\mathbf{P}}$ for some c.c.c. forcing notion $\mathbf{P}$ then e.g.

$$
\begin{aligned}
& \text { if } \aleph_{\omega \times 3}<2^{\aleph_{0}},\left\langle\aleph_{0}, \aleph_{\omega}, \aleph_{\omega+\omega}\right\rangle \rightarrow\left\langle\aleph_{\omega}, \aleph_{\omega+\omega}, \aleph_{\omega+\omega+\omega}\right\rangle \text { (see [Sh 8]), } \\
& \text { i.e., letting } \\
& \bar{\lambda}^{0}=\left\langle\left(\aleph_{0}, \aleph_{\omega}\right),\left(\aleph_{\omega}, \aleph_{\omega+\omega}\right)\right\rangle, \quad \bar{\lambda}^{1}=\left\langle\left(\aleph_{\omega}, \aleph_{\omega+\omega}\right),\left(\aleph_{\omega+\omega}, \aleph_{\omega+\omega+\omega}\right)\right\rangle,
\end{aligned}
$$

for any countable first order $\mathbf{T}$, if every finite $\mathbf{T}^{\prime} \subseteq \mathbf{T}$ has a $\bar{\lambda}^{0}$-model then $\mathbf{T}$ has a $\bar{\lambda}^{1}$-model. 
(**) $\quad\left\langle\kappa_{\xi}: \xi \leq \xi(*)\right\rangle \rightarrow\left\langle\kappa_{\xi}^{\prime}: \xi \leq \xi(*)\right\rangle$ if $\bigwedge_{\xi} \kappa_{\xi}^{+\omega} \leq \kappa_{\xi+1}$ and $\kappa_{0}^{\prime} \leq \kappa_{1}^{\prime} \leq$ $\ldots \leq \kappa_{\xi(*)}^{\prime} \leq 2^{\aleph_{0}}$ (and versions like $\left.2.11(1)\right)$.

Proof. By 2.10 if $\bar{\lambda}=\left\langle\left(\lambda_{\xi}, \kappa_{\xi}\right): \xi<\zeta(*)\right\rangle$ with $\lambda_{\xi} \geq \beth_{\omega}\left(\kappa_{\xi}\right)$ then $\operatorname{Pr}_{n}(\bar{\lambda})$ (really $\lambda_{\xi} \geq \beth_{k}\left(\kappa_{\xi}\right)$ for $k$ depending on $n$ only suffices, see [EHMR]). Now c.c.c. forcing preserves this and we apply 2.11. Similarly we can use $\theta^{+}$-c.c. forcing $\mathbf{P}$ and deal with cardinals in the interval $\left(\theta, 2^{\theta}\right)$ in $\mathbf{V}^{\mathbf{P}}$.

REMARK 2.14. We can say parallel things for the compactness of $(\exists \geq \lambda)$, for $\lambda$ singular $\leq 2^{\lambda_{0}}$ (or $\theta+|\mathcal{T}|<\lambda \leq$ number of $\theta$-branches of $\mathcal{T}$ ), e.g. we get the parallel of 2.13 .

In more details, if $\mathbf{V}_{0}=\mathbf{V}^{\mathbf{P}}$ where $\mathbf{P}$ satisfies the $\theta^{+}$-c.c. then

(*) in $\mathbf{V}_{0}^{\mathbf{P}}$, for any singular $\lambda \in\left(\theta, 2^{\theta}\right)$ such that $\mathbf{V}_{0}=$ " $\lambda$ is strong limit" we have

$(\circledast)$ the class $\left\{\left(\lambda,<, R_{\zeta} \ldots\right)_{\zeta<\theta}: R_{\zeta}\right.$ an $n_{\zeta}$-relation, $(\lambda,<)$ is $\lambda$-like $\}$ of models is $\theta$-compact, and we can axiomatize it.

There are also consistent counterexamples, see [Sh 532].

The point of proving $(*)$ is

$\otimes \quad$ for a vocabulary $\tau$ of cardinality $\leq \theta$, if we let $\mathbf{T}_{\tau}^{\text {sk }}$ be a first order theory with Skolem functions $\tau\left(T_{\tau}^{\mathrm{sk}}\right)$ (but the Skolem functions are new), then the following are equivalent for a first order $\mathbf{T} \subseteq L_{\omega, \omega}(\tau)$ :

(a) $\mathbf{T}$ has a $\lambda$-like model.

(b) The following is consistent: $\mathbf{T} \cup \mathbf{T}_{\tau}^{\mathrm{sk}} \cup\left\{\sigma\left(\ldots, x_{\eta_{l}}^{n(l)}, y_{n(l)}, \ldots\right)_{l<k}=\right.$ $\sigma\left(\ldots, x_{\nu_{l}}^{n(l)}, y_{n(l)}, \ldots\right)_{l<k} \vee \sigma\left(\ldots, x_{\eta_{l}}^{n(l)}, y_{n(l)} \ldots\right)>y_{n}: n(l)<\omega$, $\eta_{l} \in{ }^{\omega} 2$, and for some $j<\omega,\left\langle\eta_{l} \mid j: l<k\right\rangle$ is with no repetition, $\eta_{l} \uparrow j=\nu_{l}\left\lceil j, n(l)<n \Rightarrow \eta_{l}=\nu_{l}\right\} \cup\left\{\sigma\left(\ldots, x_{\eta_{l}}^{n(l)}, y_{n(l)}, \ldots\right)<y_{n}:\right.$ $n(l)<n$ and $\left.\eta_{l} \in{ }^{\omega} 2\right\}$.

\section{Finer analysis of square existence}

Definition 3.1. (1) For an $\omega$-sequence $\bar{T}=\left\langle T_{n}: n\langle\omega\rangle\right.$ of $(2,2)$-trees, we define a function degsq (square degree). Its domain is

pfap $=\operatorname{pfap}_{\bar{T}}=\left\{(u, g):(\exists n)\left(u \in\left[{ }^{n} 2\right]^{*}, g\right.\right.$ is a 2-place function from $u$ to $\omega)\}$

and its values are ordinals (or $\infty$ or -1 ). For this we define the truth value of " $\operatorname{degsq}_{\bar{T}}(u, g) \geq \alpha$ " by induction on the ordinal $\alpha$.

CASE $1, \alpha=-1$ :

$\operatorname{degsq}_{\bar{T}}(u, g) \geq-1 \quad$ iff $\quad(u, g) \in \operatorname{pfap}_{\bar{T}}$ and $\eta, \nu \in u \Rightarrow(\eta, \nu) \in T_{g(\eta, \nu)}$.

CASE 2, $\alpha$ is limit:

$$
\operatorname{degsq}_{\bar{T}}(u, g) \geq \alpha \quad \text { iff } \quad \operatorname{degsq}_{\bar{T}}(u, g) \geq \beta \text { for every } \beta<\alpha .
$$


CASE $3, \alpha=\beta+1: \operatorname{degsq}_{\bar{T}}(u, g) \geq \alpha$ iff for every $\varrho^{*} \in u$, for some $m$, $u^{*} \subseteq{ }^{m} 2, g^{*}$ and functions $h_{0}, h_{1}$, we have:

- $h_{i}: u \rightarrow u^{*}$,

- $(\forall \eta \in u) \eta \triangleleft h_{i}(\eta)$,

- $(\forall \eta \in u)\left[h_{0}(\eta)=h_{1}(\eta) \Leftrightarrow \eta \neq \varrho^{*}\right]$,

- $u^{*}=\operatorname{Rang}\left(h_{0}\right) \cup \operatorname{Rang}\left(h_{1}\right)$,

- $g^{*}$ is a 2-place function from $u^{*}$ to $\omega$,

- $g^{*}\left(h_{i}(\eta), h_{i}(\nu)\right)=g(\eta, \nu)$ for $i<2$ and $\eta, \nu \in u$,

- $\operatorname{degsq}_{\bar{T}}\left(u^{*}, g^{*}\right) \geq \beta$ (so $\left(u^{*}, g^{*}\right) \in$ pfap $)$.

(2) We define $\operatorname{degsq}_{\bar{T}}(u, g)=\alpha$ iff

for every ordinal $\beta, \operatorname{degsq}(u, g) \geq \beta \Leftrightarrow \beta \leq \alpha$

(so $\alpha=-1, \alpha=\infty$ are legal values).

(3) We define $\operatorname{degsq}(\bar{T})=\bigcup\left\{\operatorname{degsq}_{\bar{T}}(u, g)+1:(u, g) \in \operatorname{pfap}_{\bar{T}}\right\}$.

Claim 3.2. Assume $\bar{T}$ is an $\omega$-sequence of $(2,2)$-trees.

(1) For every $(u, g) \in \operatorname{pfap}_{\bar{T}}$, $\operatorname{degsq}_{\bar{T}}(u, g)$ is an ordinal, $\infty$ or -1 . Any automorphism $F$ of $\left({ }^{\omega} 2, \triangleleft\right)$ preserves this (it acts on $\bar{T}$ too, i.e.

$$
\left.\operatorname{degsq}_{\bar{T}}(u, g)=\operatorname{degsq}_{\left\langle F\left(T_{n}\right): n<\omega\right\rangle}\left(F(u), g \circ F^{-1}\right)\right) .
$$

(2) $\operatorname{degsq}(\bar{T})=\infty$ iff $\operatorname{degsq}(\bar{T}) \geq \omega_{1}$ iff there is a perfect square contained in $\bigcup_{n<\omega} \lim \left(T_{n}\right)$ iff for some c.c.c. forcing notion $\mathbf{P}, \Vdash_{\mathbf{P}}$ " $\bigcup_{n<\omega} \lim \left(T_{n}\right)$ contains a $\lambda_{\omega_{1}}\left(\aleph_{0}\right)$-square" (so those properties are absolute).

(3) If $\operatorname{degsq}(\bar{T})=\alpha(*)<\omega_{1}$ then $\bigcup_{n<\omega} \lim \left(T_{n}\right)$ contains no $\lambda_{\alpha(*)+1}\left(\aleph_{0}\right)$ square.

(4) For each $\alpha(*)<\omega_{1}$ there is an $\omega$-sequence $\bar{T}=\left\langle T_{n}: n<\omega\right\rangle$ of $(2,2)$-trees with $\operatorname{degsq}(\bar{T})=\alpha(*)$.

(5) If $\bar{T}=\left\langle T_{n}: n\langle\omega\rangle\right.$ is a sequence of $(2,2)$-trees then the existence of an $\aleph_{1}$-square in $\bigcup_{n<\omega} \lim \left(T_{n}\right)$ is absolute.

(6) Moreover, for $\alpha(*)<\omega_{1}$ we have: if $\mu<\lambda_{\alpha(*)}\left(\aleph_{0}\right)$ and $A, B$ are disjoint subsets of ${ }^{\omega} 2 \times{ }^{\omega} 2$ of cardinality $\leq \mu$, then some c.c.c. forcing notion $\mathbf{P}$ adds $\bar{T}$ as in (4) (i.e. an $\omega$-sequence $\overline{\bar{T}}=\left\langle T_{n}: n<\omega\right\rangle$ of $(2,2)$-trees with $\left.\operatorname{degsq}_{\bar{T}}(\bar{T})=\alpha(*)\right)$ such that

$$
A \subseteq \bigcup_{n<\omega} \lim \left[f\left(T_{n}\right)\right], \quad B \cap \bigcup_{n<\omega} \lim \left[f\left(T_{n}\right)\right]=\emptyset .
$$

Proof. Easy.

E.g. (3) Let $\lambda=\lambda_{\alpha(*)+1}\left(\aleph_{0}\right)$, assume $\left\{\eta_{i}: i<\lambda\right\} \subseteq{ }^{\omega} 2,[i<j \Rightarrow$ $\left.\eta_{i} \neq \eta_{j}\right]$ and $\left(\eta_{i}, \eta_{j}\right) \in \bigcup_{n} \lim \left(T_{n}\right)$, and let $\left(\eta_{i}, \eta_{j}\right) \in \lim \left(T_{g\left(\eta_{i}, \eta_{j}\right)}\right)$. For $(u, f) \in \operatorname{pfap}_{\bar{T}}$ where $u=\left\{\nu_{0}, \ldots, \nu_{k-1}\right\}$ (with no repetition, $<_{\mathrm{lx}}$-increasing) let $R_{(u, f)}=\left\{\left(\alpha_{0}, \ldots, \alpha_{k-1}\right): \alpha_{l}<\lambda\right.$ and $\nu_{l} \triangleleft \eta_{\alpha_{l}}$ for $l<k$ and $f\left(\nu_{l}, \nu_{m}\right)=$

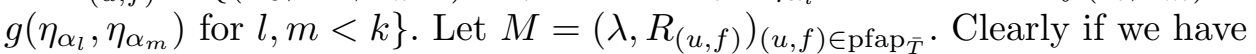


$\alpha_{0}, \ldots, \alpha_{k-1}<\lambda$ and $n$ such that $\left\langle\eta_{\alpha_{l}}\lceil n: l<k\rangle\right.$ is with no repetition, and $g\left(\eta_{\alpha_{l(1)}}, \eta_{\alpha_{l(2)}}\right)=f\left(\eta_{\alpha_{l(1)}}\left\lceil n, \eta_{\alpha_{l(2)}}\lceil n)\right.\right.$ then $R_{(u, f)}\left(\alpha_{0}, \alpha_{1}, \ldots, \alpha_{k-1}\right)$ and we can then prove

$$
\operatorname{rk}\left(\left\{\alpha_{0}, \ldots, \alpha_{k-1}\right\}, M\right) \leq \operatorname{degsq}_{\bar{T}}(u, f)
$$

(by induction on the left ordinal). But $M$ is a model with countable vocabulary and cardinality $\lambda=\lambda_{\alpha(*)+1}\left(\aleph_{0}\right)$. Hence by the definition of $\lambda_{\alpha(*)+1}$ we have $\operatorname{rk}(M) \geq \alpha(*)+1$, so $\alpha(*)+1 \leq \operatorname{rk}(M) \leq \operatorname{degsq}(\bar{T}) \leq \alpha(*)$ (by the previous sentence, an earlier sentence and a hypothesis respectively). Contradiction.

(4) Let $w_{i} \subseteq[-1, \alpha(*))$ be finite $\neq \emptyset, w_{i} \subseteq w_{i+1}$ and $[-1, \alpha(*))=$ $\bigcup_{i<\omega} w_{i}$ and let $W=\{\eta: \eta$ is a (strictly) decreasing sequence of ordinals, possibly empty $\}$. We choose, by induction on $i<\omega, n_{i}$ and an indexed set $\left\langle\left(u_{x}^{i}, f_{x}^{i}, \alpha_{x}^{i}\right)\right.$ : $\left.x \in X_{i}\right\rangle$ such that:

(a) $n_{i}<\omega, n_{0}=0, n_{i}<n_{i+1}$,

(b) $X_{i}$ is finite including $\bigcup_{j<i} X_{j}$,

(c) for $x \in X_{i}, u_{x}^{i} \subseteq{ }^{n_{i}} 2, f_{x}^{i}$ is a 2-place function from $u_{x}^{i}$ to $\omega$ and $\alpha_{x}^{i} \in w_{i}$,

(d) for some $x \in X_{i}, u_{x}^{i}=\left\{0_{n_{i}}\right\}$,

(e) $h_{i}$ is a function from $X_{i}$ into $W$ and $h_{i} \subseteq h_{i+1}$,

(f) $\left|X_{0}\right|=1$, and $h_{0}$ is constantly \langle\rangle ,

(g) if $x \in X_{i}$ then $\alpha_{x}^{i+1}=\alpha_{x}^{i}$, the function $\nu \mapsto \nu\left\lceil n_{i}\right.$ is one-to-one from $u_{x}^{i+1}$ onto $u_{x}^{i}$ and $\nu \in u_{x}^{i+1} \Rightarrow \nu \uparrow\left[n_{i}, n_{i+1}\right)=0_{\left[n_{i}, n_{i+1}\right)}$ and $\eta, \nu \in u_{x}^{i+1} \Rightarrow$ $f_{x}^{i+1}(\eta, \nu)=f_{x}^{i}\left(\eta\left\lceil n_{i}, \nu\left\lceil n_{i}\right)\right.\right.$

(h) for some $x=x_{i} \in X_{i}, \beta=\beta_{i} \in w_{i+1} \cap \alpha_{x}^{i}$ and $\varrho^{*}=\varrho_{i}^{*} \in u_{x}^{i}$ and $\Upsilon_{i} \in W$ such that $h_{i}\left(x_{i}\right) \triangleleft \Upsilon_{i}$ we can find $y=y_{i}$ such that:

$(\alpha) X_{i+1}=X_{i} \cup\left\{y_{i}\right\}, y_{i} \notin X_{i}$,

( $\beta) \alpha_{y}^{i+1}=\beta$,

$(\gamma)$ the function $\nu \mapsto \nu\left\lceil n_{i}\right.$ is a function from $u_{y}^{i+1}$ onto $u_{x}^{i}$, almost one-to-one: $\varrho^{*}$ has exactly two predecessors, say $\varrho_{1}^{y}, \varrho_{2}^{y}$, and any other $\varrho \in u_{x}^{i} \backslash\left\{\varrho^{*}\right\}$ has exactly one predecessor in $u_{y}^{i+1}$,

$(\delta)$ for $\nu, \eta \in u_{y}^{i+1}$ if $(\nu, \eta) \neq\left(\varrho_{1}^{y}, \varrho_{2}^{y}\right)$ and $(\nu, \eta) \neq\left(\varrho_{2}^{y}, \varrho_{1}^{y}\right)$ then $f_{y_{i}}^{i+1}(\eta, \nu)=f_{x}^{i}\left(\eta\left\lceil n_{i}, \nu\left\lceil n_{i}\right)\right.\right.$,

(ع) $h_{i+1}=h_{i} \cup\left\{\left(y_{i}, \Upsilon_{i}\right)\right\}$,

(i) if $x_{1}, x_{2} \in X_{i}$ and $u_{x_{1}}^{i} \cap u_{x_{2}}^{i} \neq \emptyset$ then $u_{x_{1}}^{i} \cap u_{x_{2}}^{i}=\left\{0_{n_{i}}\right\}$,

(j) if $x \in X_{i}, \beta \in w_{i} \cap \alpha_{x}^{i}, \varrho^{*} \in u_{x}^{i}$ and $h(x) \triangleleft \Upsilon \in W$ then for some $j \in(i, \omega)$ we have $x_{j}=x, \beta_{j}=\beta, \varrho^{*} \unlhd \varrho_{j}^{*}$ and $\Upsilon_{j}=\Upsilon$,

(k) the numbers $f_{y_{i}}^{i+1}\left(\varrho_{1}^{y^{1}}, \varrho_{2}^{y^{1}}\right), f_{y_{i}}^{i+1}\left(\varrho_{2}^{y^{2}}, \varrho_{1}^{y^{2}}\right)$ are distinct and do not belong to $\bigcup\left\{\operatorname{Rang}\left(f_{x}^{i}\right): x \in X_{i}\right\}$. 
There is no problem to carry out the definition. We then let

$T_{n}=\left\{(\eta, \nu): f_{x}^{i}\left(\eta^{\prime}, \nu^{\prime}\right)=n\right.$ for some $i<\omega, x \in X_{i}$ and $\eta^{\prime}, \nu^{\prime} \in u_{x}^{i}$, and $(\eta, \nu)=\left(\eta^{\prime} \uparrow l, \nu^{\prime} \uparrow l\right)$ for some $\left.l \leq n_{i}\right\}$

and $\bar{T}=\left\langle T_{n}: n<\omega\right\rangle$. Now it is straightforward to compute the rank.

(5) By the completeness theorem for $L_{\omega_{1}, \omega}(Q)$ (see Keisler [Ke71]).

(6) By the proof of 1.13 .

Now we turn to $\kappa$-Suslin sets.

Definition 3.3. Let $T$ be a $(2,2, \kappa)$-tree. Let $\operatorname{set}(T)$ be the set of all pairs $(u, f)$ such that

$$
(\exists n=n(u, f))\left[u \subseteq{ }^{n} 2 \& f: u \times u \rightarrow{ }^{n} \kappa \& \eta, \nu \in u \Rightarrow(\eta, \nu, f(\eta, \nu)) \in T\right] .
$$

We want to define $\operatorname{degsq}_{T}(x)$ for $x \in \operatorname{set}(T)$. By induction on the ordinal $\alpha$ we define when $\operatorname{degsq}_{T}(x) \geq \alpha$ :

CASE 1. $\alpha=-1$ :

$$
\operatorname{degsq}_{T}(u, f) \geq \alpha \quad \text { iff } \quad(u, f) \in \operatorname{set}(T) .
$$

CASe 2. $\alpha$ limit:

$$
\operatorname{degsq}_{T}(u, f) \geq \alpha \quad \text { iff } \quad \operatorname{degsq}_{T}(u, f) \geq \beta \text { for every } \beta<\alpha .
$$

CASE 3. $\alpha=\beta+1: \operatorname{degsq}_{T}(u, f) \geq \alpha$ iff for every $\eta^{*} \in u$, for some $m>n(u, f)$ there are $\left(u^{*}, f^{*}\right) \in \operatorname{set}(T)$ and functions $h_{0}, h_{1}$ such that $\operatorname{degsq}_{T}\left(u^{*}, f^{*}\right) \geq \beta$ and:

(i) $n\left(u^{*}, f^{*}\right)=m$,

(ii) $h_{i}$ is a function from $u$ to ${ }^{m} 2$,

(iii) $\eta \triangleleft h_{i}(\eta)$ for $i<2$,

(iv) for $\eta \in u$ we have $h_{0}(\eta) \neq h_{1}(\eta) \Leftrightarrow \eta=\eta^{*}$,

(v) for $\eta_{1} \neq \eta_{2} \in u, i<2$ we have $f\left(\eta_{1}, \eta_{2}\right) \triangleleft f^{*}\left(h_{i}\left(\eta_{1}\right), h_{i}\left(\eta_{2}\right)\right)$,

(vi) for $\eta \in u^{*}$ we have $f\left(\eta\left\lceil n, \eta\lceil n) \triangleleft f^{*}(\eta, \eta)\right.\right.$.

Lastly, $\operatorname{degsq}_{T}(u, f)=\alpha$ iff $\left[\beta \leq \alpha \Leftrightarrow \operatorname{degsq}_{T}(u, f) \geq \beta\right]$ for every ordinal $\beta$.

Also let $\operatorname{degsq}(T)=\operatorname{degsq}_{T}(\{\langle\rangle\},\{\langle\langle\rangle,\langle\rangle\rangle\})$.

Claim 3.4. (1) For a $(2,2, \kappa)$-tree $T$ and $(u, f) \in \operatorname{set}(T), \operatorname{degsq}_{T}(u, f)$ is an ordinal or infinity or $=-1$, and similarly for $\operatorname{degsq}(T)$. All are absolute. Also $\operatorname{degsq}(T) \geq \kappa^{+}$implies $\operatorname{degsq}(T)=\infty$ and similarly for $\operatorname{degsq}_{T}(u, f)$.

(2) $\operatorname{degsq}(T)=\infty$ iff $\Vdash_{\mathbf{P}}$ "prj $\lim T\left(=\left\{(\eta, \nu) \in{ }^{\omega_{2}} \times{ }^{\omega_{2}} 2\right.\right.$ : for some $\varrho \in{ }^{\omega} \kappa, \bigwedge_{n<\omega}(\eta\lceil n, \nu\lceil n, \varrho \mid n) \in T\})$ contains a perfect square" for every forcing notion $\mathbf{P}$ including a trivial one, i.e. $\mathbf{V}^{\mathbf{P}}=\mathbf{V}$ iff $\models_{\mathbf{P}} " \operatorname{prj} \lim (T)$ contains a $2^{\aleph_{0}}$-square" for the forcing notion $\mathbf{P}$ which is adding $\lambda$ Cohen reals for $\lambda=\lambda_{\kappa^{+}}^{3}(\kappa)$ iff for some $\mathbf{P}, \Vdash_{\mathbf{P}} " \operatorname{prj} \lim (T)$ contains a $\lambda_{\kappa^{+}}\left(\aleph_{0}\right)$ square". 
(3) If $\alpha(*)=\operatorname{degsq}(T)<\kappa^{+}$, then $\operatorname{prj} \lim (T)$ contains no $\lambda_{\alpha(*)+1}(\kappa)$ square.

Proof. (1) Easy.

(2) Assume that $\operatorname{degsq}(T)=\infty$, and note that $\alpha^{*}=\left\{\operatorname{degsq}_{T}(u, f)\right.$ : $(u, f) \in \operatorname{set}(T)$ and $\left.\operatorname{degsq}_{T}(u, f)<\infty\right\} \backslash\{\infty\}$ is an ordinal so $(u, f) \in$ $\operatorname{set}(T) \& \operatorname{degsq}_{T}(u, f) \geq \alpha^{*} \Rightarrow \operatorname{degsq}_{T}(u, f)=\infty$ (in fact, any ordinal $\alpha \geq$ $\sup \left\{\operatorname{degsq}_{T}(u, f)+1:(u, f) \in \operatorname{set}(T)\right\}$ will do). Let $\operatorname{set}^{\infty}(T)=\{(u, f) \in$ $\left.\operatorname{set}(T): \operatorname{degsq}_{T}(u, f)=\infty\right\}$. Now

$(*)_{1} \quad$ There is $(u, f) \in \operatorname{set}^{\infty}(T)$.

$(*)_{2} \quad$ For every $(u, f) \in \operatorname{set}^{\infty}(T)$ and $\varrho \in u$ we can find $\left(u^{+}, f^{+}\right) \in \operatorname{set}^{\infty}(T)$ and for $e=1,2, h_{e}: u \rightarrow u^{+}$such that $(\forall \eta \in u)\left(\eta \triangleleft h_{e}(\eta)\right),(\forall \eta, \nu \in u)$ $\left(f(\eta, \nu) \triangleleft f^{+}\left(h_{e}(\eta), h_{e}(\nu)\right),(\forall \eta \in u)\left[h_{1}(\eta)=h_{2}(\eta) \Leftrightarrow \eta=\varrho\right]\right.$.

[Why? As $\operatorname{degsq}_{T}(u, f)=\infty$ it is $\geq \alpha^{*}+1$ so by the definition we can find $\left(u^{+}, f^{+}\right)$and $h_{1}, h_{2}$ as above but only with $\operatorname{degsq}_{T}\left(u^{+}, f^{+}\right) \geq \alpha^{*}$, and this implies $\operatorname{degsq}_{T}\left(u^{+}, f^{+}\right)=\infty$.]

$(*)_{3} \quad$ For every $(u, f) \in \operatorname{set}^{\infty}(T)$ with $u=\left\{\eta_{\varrho}: \varrho \in{ }^{n} 2\right\} \subseteq{ }^{\left(n_{1}\right)} 2$ (no repetition) we can find $n_{2}>n_{1}$ and $\left(u^{+}, f^{+}\right) \in \operatorname{set}^{\infty}(T)$ with $u^{+}=$ $\left\{\eta_{\varrho}: \varrho \in^{n+1} 2\right\} \subseteq{ }^{\left(n_{2}\right)} 2$ (no repetitions) such that:

(i) $\varrho \in{ }^{n+1} 2 \Rightarrow \eta_{\varrho \cap n} \triangleleft \eta_{\varrho}$,

(ii) for $\varrho_{1}, \varrho_{2} \in{ }^{n+1} 2, \varrho_{1}\left\lceil n \neq \varrho_{2}\left\lceil n \Rightarrow f\left(\eta_{\varrho_{1}\lceil n}, \eta_{\varrho_{2}\lceil n}\right) \triangleleft f^{+}\left(\eta_{\varrho_{1}}, \eta_{\varrho_{2}}\right)\right.\right.$,

(iii) for $\varrho \in{ }^{n+1} 2, f\left(\eta_{\varrho}\left\lceil n, \eta_{\varrho}\lceil n) \triangleleft f\left(\eta_{\varrho}, \eta_{\varrho}\right)\right.\right.$.

[Why? Repeat $(*)_{2} 2^{n}$ times.]

So we can find $\left\langle\eta_{\varrho}: \eta \in{ }^{n} 2\right\rangle$ and $f_{n}$ by induction on $n$ such that $\left\langle\eta_{\varrho}\right.$ : $\left.\varrho \in{ }^{n} 2\right\rangle$ is with no repetition, $\operatorname{degsq}_{T}\left(\left\{\eta_{\varrho}: \varrho \in{ }^{n} 2\right\}, f_{n}\right)=\infty$, and for each $n$ clauses (i)-(iii) of $(*)_{3}$ hold, i.e. $\varrho_{1}, \varrho_{2} \in{ }^{n+1} 2, \varrho_{1}\left\lceil n \neq \varrho_{2}\lceil n \Rightarrow\right.$ $f_{n}\left(\eta_{\varrho_{1}\lceil n}, \eta_{\varrho_{2}\lceil n}\right) \triangleleft f_{n+1}\left(\eta_{\varrho_{1}}, \eta_{\varrho_{2}}\right)$ and for $\varrho \in^{n+1} 2$ we have $f_{n}\left(\eta_{\varrho\lceil n}, \eta_{\varrho \nmid n}\right) \triangleleft$ $f_{n+1}\left(\eta_{\varrho}, \eta_{\varrho}\right)$ and of course $\left\{\eta_{\varrho}: \varrho \in{ }^{m} 2\right\} \subseteq{ }^{\left(k_{n}\right)} 2$ with $k_{n}<k_{n+1}<\omega$.

So we have proved that the first clause implies the second (about the forcing: the $\operatorname{degsq}(T)=\infty$ is absolute so holds also in $\mathbf{V}^{\mathbf{P}}$ for any forcing notion $\mathbf{P})$. Trivially the second clause implies the third and fourth. So assume the third clause and we shall prove the first. By 1.8, $\lambda_{\kappa^{+}}^{2}(\kappa)$ is well defined (e.g. $\left.\leq \beth_{\kappa^{+}}\right)$, but $\lambda_{\kappa^{+}}^{3}(\kappa)=\lambda_{\kappa^{+}}^{2}(\kappa)$ by 1.5(3). Let $\mathbf{P}$ be the forcing notion adding $\lambda_{\kappa^{+}}^{2}(\kappa)$ Cohen reals. By 1.11(2) in $\mathbf{V}^{\mathbf{P}}, \lambda_{\kappa^{+}}(\kappa) \leq \lambda_{\kappa^{+}}^{2}(\kappa) \leq 2^{\aleph_{0}}$, and so there are pairwise disjoint $\eta_{i} \in{ }^{\omega} 2$ for $i<\lambda_{\kappa^{+}}(\kappa)$ such that $\left(\eta_{i}, \eta_{j}\right) \in \operatorname{prj} \lim (T)$ for $i, j<\lambda_{\kappa^{+}}(\kappa)$. Lastly, we prove that the fourth clause implies the first in $\mathbf{V}^{\mathbf{P}}$. By part (3) of the claim proved below, for every $\alpha<\kappa^{+}$we get $\neg[\alpha=\operatorname{degsq}(T)]$ as $\lambda_{\alpha}(\kappa) \leq \lambda_{\kappa^{+}}(\kappa)$. Hence $\operatorname{degsq}(T) \geq \kappa^{+}$, but by part (1) this implies $\operatorname{degsq}(T)=\infty$.

(3) Just like the proof of $3.2(3)$. 
We shall prove in [Sh 532]

Claim 3.5. Assume $\alpha(*)<\kappa^{+}$and $\lambda<\lambda_{\alpha(*)}(\kappa)$.

(1) For some c.c.c. forcing notion $\mathbf{P}$, in $\mathbf{V}^{\mathbf{P}}$ there is a $\kappa$-Suslin subset $A=\operatorname{prj} \lim (T)$ (where $T$ is a $(2,2, \kappa)$-tree) such that

A contains a $\lambda$-square but $\operatorname{degsq}(T) \leq \alpha(*)$.

(2) For given $B \subseteq\left({ }^{\omega} 2 \times{ }^{\omega} 2\right)^{\mathbf{V}}$ of cardinality $\leq \lambda$ we can replace $(*)$ by

$$
A \cap\left({ }^{\omega} 2 \times{ }^{\omega} 2\right)^{\mathbf{V}}=B \quad \text { but } \quad \operatorname{degsq}(T) \leq \alpha(*) .
$$

REMARK 3.6. The following says in fact that "colouring of pairs is enough", say for the Hanf number of $L_{\omega_{1}, \omega}$ below the continuum; for clarification see 3.8.

Claim 3.7 (MA). Assume $\lambda<2^{\aleph_{0}}$ and $\alpha(*)<\omega_{1}$ is a limit ordinal, $\lambda<\mu:=\lambda_{\alpha(*)}\left(\aleph_{0}\right)$. Then for some symmetric 2-place function $F$ from $\lambda$ to $\omega$ we have

$(*)_{\lambda, \mu, F} \quad$ for no 2-place (symmetric) function $F^{\prime}$ from $\mu$ to $\omega$ do we have:

$(* *)$ for every $n<\omega$ and pairwise distinct $\beta_{0}, \ldots, \beta_{n-1}<\mu$ there are pairwise distinct $\alpha_{0}, \ldots, \alpha_{n-1}<\lambda$ such that

$$
\bigwedge_{k<l<n} F^{\prime}\left(\beta_{k}, \beta_{l}\right)=F\left(\alpha_{k}, \alpha_{l}\right) .
$$

Remark 3.8. (1) This is close to Gilchrist-Shelah [GcSh 491].

(2) The proof of 3.7 says that letting $R_{n}=\{(\alpha, \beta): F(\alpha, \beta)=n\}$ and $N=\left(\lambda, R_{n}\right)_{n<\omega}$, we have $\operatorname{rk}(N)<\alpha(*)$.

(3) So 3.7 improves $\S 2$ by saying that the examples for the Hanf number of $L_{\omega_{1}, \omega}$ below the continuum being large can be very simple, speaking only about "finite patterns" of colouring pairs by countably many colours.

P r o of (of Claim 3.7). Let $M$ be a model of cardinality $\lambda$ with a countable vocabulary and $\operatorname{rk}^{1}(M)<\alpha(*)$. We can assume that it has universe $\lambda$, relation $<$ and individual constants $c_{\alpha}$ for $\alpha \leq \omega$. Let $k^{M}, \varphi^{M}$ be as in the proof of 1.13 .

Let $\mathbf{P}$ be the set of triples $(u, f, \mathbf{w})$ such that:

(a) $u$ is a finite subset of $\lambda$,

(b) $f$ is a symmetric 2-place function from $u$ to $\omega$,

(c) $\mathbf{w}$ is a family of nonempty subsets of $u$ such that

(d) if $\alpha \in u$ then $\{\alpha\} \in \mathbf{w}$,

(e) if $w=\left\{\alpha_{0}, \ldots, \alpha_{n-1}\right\} \in \mathbf{w}$ (increasing enumeration), $k=k^{M}(w)$, $\alpha \in u \backslash w$ and $(\forall l)\left[l<n \& l \neq k \Rightarrow f\left(\alpha_{l}, \alpha\right)=f\left(\alpha_{l}, \alpha_{k}\right)\right]$ then $w \cup\{\alpha\}$ belongs to $\mathbf{w},(\forall m \neq k)\left(\alpha<\alpha_{m} \Leftrightarrow \alpha_{k}<\alpha_{m}\right)$ and $k=k^{M}\left(w \cup\{\alpha\} \backslash\left\{\alpha_{k}\right\}\right)$ and $M \models \varphi^{M}(w)\left[\alpha_{0}, \ldots, \alpha_{k-1}, \alpha, \alpha_{k+1}, \ldots, \alpha_{n-1}\right]$, 
(f) if $w^{i}=\left\{\alpha_{0}^{i}, \ldots, \alpha_{n-1}^{i}\right\} \subseteq u$ (increasing enumeration, so with no repetition), $i=0,1$, and $(\forall l<k<n)\left[f\left(\alpha_{l}^{0}, \alpha_{k}^{0}\right)=f\left(\alpha_{l}^{1}, \alpha_{k}^{1}\right)\right]$ then $w^{0} \in \mathbf{w}$ $\Leftrightarrow w^{1} \in \mathbf{w}$ and if $w^{i} \in \mathbf{w}$ then $\varphi^{M}\left(w^{0}\right)=\varphi^{M}\left(w^{1}\right), k^{M}\left(w^{0}\right)=k^{M}\left(w^{1}\right)$ and $\operatorname{rk}^{1}\left(w^{0}, M\right)=\operatorname{rk}^{1}\left(w^{1}, M\right)$.

The order is the natural one.

It is easy to check that:

$\oplus_{1} \quad \mathbf{P}$ satisfies the c.c.c.

$\oplus_{2} \quad$ for every $\alpha<\lambda, \mathcal{L}_{\alpha}=\{(u, f, \mathbf{w}) \in \mathbf{P}: \alpha \in u\}$ is dense.

Hence there is a directed $G \subseteq \mathbf{P}$ not disjoint from $\mathcal{L}_{\alpha}$, for every $\alpha<\lambda$. Let $F=\bigcup\{f:(u, f, \mathbf{w}) \in G$ for some $u, \mathbf{w}\}$. We shall show that it is as required. Clearly $F$ is a symmetric 2-place function from $\lambda$ to $\omega$; so the only thing that can fail is if there is a symmetric 2-place function $F^{\prime}$ from $\mu$ to $\omega$ such that $(* *)$ of 3.7 holds. By the compactness theorem for propositional logic, there is a linear order $<^{*}$ of $\mu$ such that

$(*)^{\prime} \quad$ for every $n<\omega$ and $\beta_{0}<^{*} \ldots<^{*} \beta_{n-1}$ from $\mu$ there are $\alpha_{0}<\ldots<$ $\alpha_{n-1}<\lambda$ such that $\bigwedge_{k<l<n} F^{\prime}\left(\beta_{k}, \beta_{l}\right)=F\left(\alpha_{k}, \alpha_{l}\right)$.

Let

$$
\mathbf{W}=\bigcup\{\mathbf{w}:(u, f, \mathbf{w}) \in G \text { for some } u, f\} .
$$

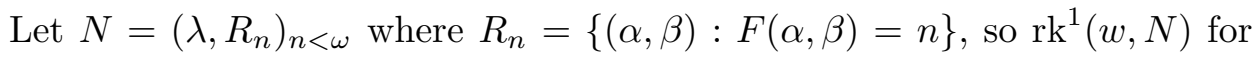
$w \in[\lambda]^{*}$ is well defined; in fact, we can restrict ourselves to formulas of the form $\varphi\left(x_{0}, \ldots, x_{n-1}\right)=\bigwedge_{l<k<n} F\left(x_{l}, x_{k}\right)=c_{m(l, k)}$. Let $N^{\prime}=\left(\mu, R_{n}^{\prime}\right)_{n<\omega}$ where $R_{n}^{\prime}=\left\{(\alpha, \beta): F^{\prime}(\alpha, \beta)=n\right\}$. Now first note that

$\otimes_{1}$

$$
\text { if } w \in \mathbf{W} \text { then } \operatorname{rk}^{1}(w, N) \leq \operatorname{rk}^{1}(w, M)
$$

(this is the role of clause (e) in the definition of $\mathbf{P}$; we prove it by induction on $\operatorname{rk}^{1}(w, M)$ using the same "witness" $\left.k^{M}\right)$. Secondly,

$$
\{\alpha\} \in \mathbf{W} \quad \text { for } \alpha<\lambda
$$

(this is the role of clause (d) in the definition of $\mathbf{P}$ ). Hence we conclude (by $\left.1.1(2), \otimes_{1}, \otimes_{2}\right)$ that

$$
\operatorname{rk}^{1}(N)<\alpha(*)
$$

Lastly,

$\otimes_{4} \quad$ if $\alpha_{0}<^{*} \ldots<^{*} \alpha_{m-1}$ are $<\mu, \beta_{0}<\ldots<\beta_{m-1}<\lambda, \bigwedge_{k<l<m} F^{\prime}\left(\alpha_{k}, \alpha_{l}\right)$ $=F\left(\beta_{k}, \beta_{l}\right)$ and $\left\{\beta_{0}, \ldots, \beta_{m-1}\right\} \in \mathbf{W}$ then $\operatorname{rk}^{1}\left(\left\{\alpha_{0}, \ldots, \alpha_{m-1}\right\}, N^{\prime}\right) \leq$ $\operatorname{rk}^{1}\left(\left\{\beta_{0}, \ldots, \beta_{m-1}\right\}, N\right)$

(again it can be proved by induction on $\operatorname{rk}^{1}\left(\left\{\beta_{l}, \ldots, \beta_{m-1}\right\}, N\right)$, the choice of $N^{\prime}$ and our assumption toward a contradiction that $(* *)$ of the claim holds). Now by $\otimes_{3}, \otimes_{4}$ and 1.1(2) (and $\otimes_{2}$ ) we have $\operatorname{rk}^{1}\left(N^{\prime}\right) \leq \operatorname{rk}^{1}(N)<\alpha(*)$, but this contradicts $\left\|N^{\prime}\right\|=\mu=\lambda_{\alpha(*)}\left(\aleph_{0}\right)$. 
Claim 3.9 (MA). If $\lambda, \mu, F$ are as in 3.7 (i.e. $(*)_{\lambda, \mu, F}$ holds) then some Borel set $B \subseteq \omega_{2} \times{ }^{\omega} 2$ (actually of the form $\bigcup_{n<\omega} \lim \left(T_{n}\right)$ ) has a $\lambda$-square but no $\mu$-square.

REMARK 3.10. (1) The converse holds too, of course.

(2) We can use "all $\lambda<\mu$ ".

Proof (of Claim 3.9). We can assume $\operatorname{cf}(\lambda)>\aleph_{0}$ (otherwise combine $\omega$ examples). Let $F$ be a symmetric 2-place function from $\lambda$ to $\omega$ such that $(*)_{\lambda, \mu, F}$. For simplicity let $f^{*}: \omega \rightarrow \omega$ be such that $(\forall n)\left(\exists^{\infty} m\right)\left(f^{*}(m)=n\right)$. We define a forcing notion $\mathbf{P}$ as in 1.13 except that we require in addition for $p \in \mathbf{P}$ :

$\otimes_{1} \quad f^{*}\left(g^{p}(\alpha, \beta)\right)=F(\alpha, \beta)$,

$\otimes_{2} \quad$ if $\alpha^{\prime} \neq \beta^{\prime}, \alpha^{\prime \prime} \neq \beta^{\prime \prime}$ are from $u^{p}, k<\omega, \eta_{\alpha^{\prime}}^{p} \uparrow k=\eta_{\alpha^{\prime \prime}}^{p}\left\lceil k \neq \eta_{\beta^{\prime}}^{p} \uparrow k=\right.$ $\eta_{\beta^{\prime \prime}}^{p}\left\lceil k\right.$ both not constantly 1 then $F\left(\alpha^{\prime}, \beta^{\prime}\right)=F\left(\alpha^{\prime \prime}, \beta^{\prime \prime}\right)$,

$\otimes_{3} \quad$ if $\eta, \nu \in{ }^{n[p]} 2$ then for at most one $m<m[p]$ we have $(\eta, \nu) \in t_{m}^{p}$,

$\otimes_{4} \quad$ if $n<\omega, n>1, \eta_{0}<_{\mathrm{lx}} \ldots<_{\mathrm{lx}} \eta_{n-1}$ are pairwise distinct members of ${ }^{n(p)} 2$ and $k<l \Rightarrow\left(\eta_{k}, \eta_{l}\right) \in t_{g(k, l)}^{p}$ then for some pairwise distinct $\alpha_{0}, \ldots, \alpha_{n-1}$ from $u\left[p^{n}\right]$, we have

$$
\bigwedge_{k<l<n} f^{*}(h(k, l))=F\left(\alpha_{k}, \alpha_{l}\right)
$$

We have

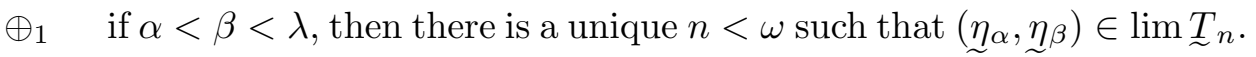

Thus $\bigcup_{n \in \omega} T_{n}$ contains a $\lambda$-square. In proving that it does not contain a $\mu$-square we apply $(*)_{\lambda, \mu, F}$. For this the crucial fact is

$\oplus_{2} \quad$ if $n<\omega, \eta_{0}, \ldots, \eta_{n-1} \in{ }^{\omega} 2$ are distinct, $\left(\eta_{k}, \eta_{l}\right) \in \lim \left(T_{h(k, l)}\right)$ then for some pairwise distinct $\alpha_{0}, \ldots, \alpha_{n-1}<\lambda$,

$$
\bigwedge_{k<l<n} f^{*}(h(k, l))=F\left(\alpha_{k}, \alpha_{l}\right) .
$$

Instead of " $\mathcal{I}_{\alpha}^{3}$ is dense" it is enough to show

$\oplus_{3} \quad$ for some $p \in \mathbf{P}, p \Vdash$ "the number of $\alpha<\lambda$ such that $\alpha \in u^{q}$ for some $q \in G_{\mathbf{P}}$ is $\lambda "$.

Similarly we can show

Claim 3.11 (MA). Assume $\aleph_{0}<\lambda<2^{\aleph_{0}}$. Then the following are equivalent:

(a) For some symmetric 2-place functions $F_{\mu}$ from $\mu$ to $\omega($ for $\mu<\lambda)$ we have

$(*)_{\left\langle F_{\mu}: \mu<\lambda\right\rangle}$ for no 2-place function $F^{\prime}$ from $\lambda$ to $\omega$ do we have: 
(**) for every $n<\omega$ and pairwise distinct $\beta_{0}, \ldots, \beta_{n-1}<\lambda$ there are $\mu<\lambda$ and pairwise distinct $\alpha_{0}, \ldots, \alpha_{n-1}<\mu$ such that

$$
\bigwedge_{k<l<n} F^{\prime}\left(\beta_{k}, \beta_{l}\right)=F_{\mu}\left(\alpha_{k}, \alpha_{l}\right) .
$$

(b) Some Borel subset of ${ }^{\omega} 2 \times{ }^{\omega} 2$ contains a $\mu$-square iff $\mu<\lambda$ (in fact, $B$ is an $F_{\sigma}$ set).

4. Rectangles. Simpler than squares are rectangles: subsets of ${ }^{\omega} 2 \times{ }^{\omega} 2$ of the form $X_{0} \times X_{1}$, characterized by pairs of cardinals $\left\langle\lambda_{0}, \lambda_{1}\right\rangle$ where $\lambda_{l}=\left|X_{l}\right|$. So we would like to define ranks and cardinals which characterize their existence just as $\operatorname{rk}^{l}(w, M ; \kappa), \lambda_{\alpha}^{l}(\kappa), \operatorname{degsq}_{\bar{T}}(-), \operatorname{degsq}_{T}(-)$ have done for squares. Though the demands are weaker, the formulation is more cumbersome: we need two "kinds" of variables, one corresponding to $\lambda_{0}$ and one to $\lambda_{1}$. So the models have two distinguished predicates, $R_{0}, R_{1}$ (corresponding to $X_{0}, X_{1}$ respectively), and in the definition of rank we connect only elements from distinct sides (in fact, in $\S 3$ we already concentrate on 2-place relations explaining not much is lost). This is very natural as except for inequality nothing connects two members of $X_{0}$ or two members of $X_{1}$.

Definition 4.1. We define $\operatorname{Prrc}_{\alpha}^{l}\left(\lambda_{1}, \lambda_{2} ;<\kappa, \theta_{0}, \theta_{1}\right), \operatorname{rkrc}^{l}\left(\left(w_{1}, w_{2}\right), M\right.$, $\left.\lambda_{1}, \lambda_{2} ; \kappa, \theta_{0}, \theta_{1}\right)$ as in 1.1 (but $w_{l} \in\left[R_{l}^{M}\right]^{*}$ and $\left|R_{l}^{M}\right|=\lambda_{l}$ ), replacing rk by rkrc etc. Let $\bar{\lambda}=\left(\lambda_{1}, \lambda_{2}\right)$ and $\bar{w}=\left(w_{1}, w_{2}\right)$.

(1) For $l<6$, cardinals $\bar{\lambda}=\left(\lambda_{1}, \lambda_{2}\right)$ with $\lambda_{1}, \lambda_{2} \geq \kappa$ and $\bar{\theta}=\left(\theta_{0}, \theta_{1}\right)$ with $\theta_{0} \leq \theta_{1}<\lambda_{1}, \lambda_{2}$, and an ordinal $\alpha$, let $\operatorname{Prrc}_{\alpha}^{l}(\bar{\lambda} ;<\kappa, \bar{\theta})$ mean that for every model $M$ with vocabulary of cardinality $\leq \theta_{0}$ such that $\bigwedge_{i=1}^{2}\left|R_{i}^{M}\right|=\lambda_{i}$, $R_{1}^{M} \cap R_{2}^{M}=\emptyset, F^{M}$ is a 2-place function with range included in $\theta_{1}=Q^{M}$, we have $\operatorname{rkrc}^{l}(M ;<\kappa) \geq \alpha$ (defined below).

Let $\operatorname{NPrrc}_{\alpha}^{l}(\bar{\lambda} ;<\kappa, \bar{\theta})$ be the negation. Instead of $<\kappa^{+}$we may write $\kappa$; if $\kappa=\theta_{0}^{+}$we may omit $\theta_{0}$; if $\theta_{0}=\aleph_{0}, \kappa=\aleph_{1}$, we may omit them. We may write $\theta_{0}, \theta_{1}$ instead of $\bar{\theta}=\left(\theta_{0}, \theta_{1}\right)$ and similarly for $\bar{\lambda}$. Lastly, we let $\lambda \operatorname{rc}_{\alpha}^{l}(\kappa, \bar{\theta})=\min \left\{\lambda: \operatorname{Prrc}_{\alpha}^{l}(\lambda, \lambda ;<\kappa, \bar{\theta})\right\}$.

(2) For a model $M$,

$$
\begin{aligned}
& \operatorname{rkrc}^{l}(M ;<\kappa)=\sup \left\{\operatorname{rkrc}^{l}(\bar{w}, M ;<\kappa)+1: \bar{w}=\left\langle w_{1}, w_{2}\right\rangle\right. \text { where } \\
& w_{i} \subseteq R_{i}^{M} \text { are finite nonempty for } i=1,2 \text { and } \\
& \left.\left(\exists c \in Q^{M}\right)(\forall a, b)\left[a \in w_{1} \& b \in w_{2} \Rightarrow F(a, b)=c\right]\right\}
\end{aligned}
$$

where rkrc is as defined in part (3) below.

(3) For a model $M$ and

$$
\bar{w} \in[M]^{\otimes}:=\left\{\bar{u}: \bar{u}=\left(u_{1}, u_{2}\right), u_{i} \subseteq R_{i}^{M}\right. \text { are finite nonempty and }
$$

$$
\left.(\exists c)(\forall a, b)\left[a \in u_{1} \& b \in u_{2} \Rightarrow F(a, b)=c\right]\right\}
$$


we shall define the truth value of $\operatorname{rkrc}^{l}(\bar{w}, M ;<\kappa) \geq \alpha$ by induction on the ordinal $\alpha$ (for $l=0,1, \kappa$ can be omitted). If we write $w$ instead of $w_{1}, w_{2}$ we mean $w_{1}=w \cap R_{1}^{M}, w_{2}=w \cap R_{2}^{M}$ (here $R_{1}^{M} \cap R_{2}^{M}=\emptyset$ helps). Then we can note

$(*)_{0} \quad \alpha \leq \beta \& \operatorname{rkrc}^{l}(\bar{w}, M ;<\kappa) \geq \beta \Rightarrow \operatorname{rkrc}^{l}(\bar{w}, M ;<\kappa) \geq \alpha$,

$(*)_{1} \quad \operatorname{rkrc}^{l}(\bar{w}, M ;<\kappa) \geq \delta(\delta$ limit $) \quad$ iff $\bigwedge_{\alpha<\delta} \operatorname{rkrc}^{l}(\bar{w}, M ;<\kappa) \geq \alpha$,

$(*)_{2} \quad \operatorname{rkrc}^{l}(\bar{w}, M ;<\kappa) \geq 0 \quad$ iff $\quad \bar{w} \in[M]^{\otimes}$.

So we can define $\operatorname{rkrc}^{l}(\bar{w}, M ;<\kappa)$ to be the maximal $\alpha$ such that $\operatorname{rkrc}^{l}(\bar{w}, M$; $<\kappa) \geq \alpha$, and $\infty$ if this holds for every $\alpha$ (and $-1 \operatorname{if}_{\operatorname{rkrc}}^{l}(\bar{w}, M ;<\kappa) \nsupseteq 0$ ).

Now the inductive definition of $\operatorname{rkrc}^{l}(\bar{w}, M ;<\kappa) \geq \alpha$ was already done above for $\alpha=0$ and $\alpha$ limit, so for $\alpha=\beta+1$ we let

$(*)_{3} \quad \operatorname{rkrc}^{l}(\bar{w}, M ;<\kappa) \geq \beta+1$ iff (letting $w=w_{1} \cup w_{2}, n=|w|, w=$ $\left.\left\{a_{0}, \ldots, a_{n-1}\right\}\right)$, for every $k<n$ and quantifier free formula

$$
\begin{aligned}
& \varphi\left(x_{0}, \ldots, x_{n-1}\right)= \\
& \bigwedge_{i<j} x_{i} \neq x_{j} \& \bigwedge\left\{R_{1}\left(x_{i}\right) \wedge R_{2}\left(x_{j}\right) \wedge \varphi_{i, j}\left(x_{i}, x_{j}\right): R_{1}\left(a_{i}\right) \& R_{2}\left(a_{j}\right)\right\}
\end{aligned}
$$

(in the vocabulary of $M$ ) for which $M \models \varphi\left[a_{0}, \ldots, a_{n-1}\right]$ we have:

CASE 1: $l=1$. There are $a_{m}^{i} \in M$ for $m<n, i<2$ such that:

(a) $\operatorname{rkrc}^{l}\left(\left\{a_{m}^{i}: i<2, m<n\right\}, M ;<\kappa\right) \geq \beta$,

(b) $M \models \varphi\left[a_{0}^{i}, \ldots, a_{n-1}^{i}\right]$ (for $i=1,2$ ), so there is no repetition in $a_{0}^{i}, \ldots, a_{n-1}^{i}$ and $\left[a_{m}^{i} \in R_{j}^{M} \Leftrightarrow a_{m} \in R_{j}^{M}\right]$ for $j=1,2$,

(c) $a_{k}^{0} \neq a_{k}^{1}$ but if $m<n$ and $\left(a_{m} \in R_{1}^{M} \Leftrightarrow a_{k} \notin R_{1}^{M}\right)$ then $a_{m}^{0}=a_{m}^{1}$

(d) if $a_{m_{1}} \in R_{1}^{M}$ and $a_{m_{2}} \in R_{2}^{M}$ then for any $i, j(\in\{1,2\})$ we have $F^{M}\left(a_{m_{1}}^{i}, a_{m_{2}}^{j}\right)=F^{M}\left(a_{m_{1}}, a_{m_{2}}\right)$.

CASE 2: $l=0$. As for $l=1$ but in addition

(e) $\bigwedge_{m} a_{m}=a_{m}^{0}$.

CASE 3: $l=3$. The definition is like case 1 but $i<\kappa$; i.e. there are $a_{m}^{i} \in M$ for $m<n, i<\kappa$ such that:

(a) for $i<j<\kappa$ we have $\operatorname{rkrc}^{l}\left(\left\{a_{m}^{i}, a_{m}^{j}: m<n\right\}, M ;<\kappa\right) \geq \beta$,

(b) $M \models \varphi\left[a_{0}^{i}, \ldots, a_{n-1}^{i}\right]$ (for $i<\kappa$; so there are no repetitions in $\left.a_{0}^{i}, \ldots, a_{n-1}^{i}\right)$,

(c) for $i<j<\kappa, a_{k}^{i} \neq a_{k}^{j}$ but if $m<n$ and $\left(a_{m} \in R_{1}^{M} \Leftrightarrow a_{k} \notin R_{1}^{M}\right)$ then $a_{m}^{i}=a_{m}^{j}$,

(d) if $a_{m_{1}} \in R_{1}^{M}$ and $a_{m_{2}} \in R_{2}^{M}$ then for any $i, j, F^{M}\left(a_{m_{1}}^{i}, a_{m_{2}}^{j}\right)=$ $F^{M}\left(a_{m_{1}}, a_{m_{2}}\right)$. 
CASE $4: l=2$. Like case 3 but in addition

(e) $a_{m}=a_{m}^{0}$ for $m<n$.

CASE 5: $l=5$. Like case 3 except that we replace clause (a) by

(a) ${ }^{-}$for every function $H$ with $\operatorname{Dom}(H)=\kappa$ and $|\operatorname{Rang}(H)|<\kappa$, for some $i<j<\kappa$ we have $H(i)=H(j)$ and

$$
\operatorname{rkrc}^{l}\left(\left\{a_{m}^{i}, a_{m}^{j}: m<n\right\}, M ;<\kappa\right) \geq \beta .
$$

CASE 6: $l=4$. Like case 4 with (a) ${ }^{-}$instead (a).

(4) For $M$ as above and $c \in Q^{M}$ we define $\operatorname{rkrc}^{l}(M, c ;<\kappa)$ as

$$
\begin{aligned}
\sup \left\{\operatorname{rkrc}^{l}(\bar{w}, M ;<\kappa)+1:\right. & \bar{w} \in[M]^{\otimes} \text { and } \\
& \left.\left(\forall a \in w_{1}\right)\left(\forall b \in w_{2}\right)[F(a, b)=c]\right\} .
\end{aligned}
$$

(5) Let $\operatorname{Prrd}_{\alpha}^{l}(\bar{\lambda}, \kappa, \bar{\theta})$ mean $\operatorname{rkrc}^{l}(M, c ;<\kappa, \bar{\theta}) \geq \alpha$ for every $M$ for some $c \in M$ when $M$ is such that $\left|R_{1}^{M}\right|=\lambda_{1},\left|R_{2}^{M}\right|=\lambda_{2},|\tau(M)| \leq \theta_{0}$, $F^{M}: R_{1}^{M} \times R_{2}^{M} \rightarrow Q^{M}$ and $\left|Q^{M}\right|=\theta_{1}$. Let $\operatorname{NPrrd}_{\alpha}^{l}(\bar{\lambda}, \kappa, \bar{\theta})$ mean its negation and $\lambda \operatorname{rd}_{\alpha}^{l}(\kappa, \bar{\theta})$ be the minimal $\lambda$ such that $\operatorname{Prrd}_{\alpha}^{l}(\lambda, \lambda, \kappa, \bar{\theta})$.

REMARK 4.2. The reader may wonder why in addition to Prrc we use the variant Prrd. The point is that for the existence of the rectangle $X_{1} \times X_{2}$ with $F \uparrow\left(X_{1} \times X_{2}\right)$ constantly $c^{*}$, this constant plays a special role. So in our main claim 4.6, to get a model as there, we need to choose it, one out of $\theta_{1}$, but the other choices are out of $\kappa$. So though the difference between the two variants is small (see 4.5 below) we actually prefer the Prrd version.

Claim 4.3. The parallels of 1.2 (+statements in 1.1), also 1.3, 1.5(2), 1.6, 1.10 hold.

Claim 4.4. (1) If $w_{i} \in\left[R_{i}^{M}\right]^{*}$ for $i=1,2$ then

$$
\omega \times \operatorname{rkrc}^{l}\left(\left\langle w_{1}, w_{2}\right\rangle, M ; \kappa\right) \geq \operatorname{rk}^{l}\left(w_{1} \cup w_{2}, M ; \kappa\right) .
$$

(2) If $R_{1}^{M}=R_{2}^{M}$ (abuse of notation) then $\operatorname{rkrc}^{l}(M ;<\kappa) \geq \operatorname{rk}^{l}(M ; \kappa)$.

(3) If $\lambda_{1}=\lambda_{2}=\lambda$ then $\operatorname{Pr}_{\alpha}(\lambda, \kappa) \Rightarrow \operatorname{Prr}_{\alpha}\left(\lambda_{1}, \lambda_{2} ; \kappa, \kappa\right)$.

CLAIM 4.5. $\lambda \operatorname{rd}_{\alpha}^{l}(\kappa, \theta)=\lambda \operatorname{rc}_{\alpha}^{l}(\kappa, \theta)$ if $\alpha$ is a successor ordinal or $\operatorname{cf}(\alpha)$ $>\theta$.

Claim 4.6. Assume $\kappa \leq \theta<\lambda_{1}, \lambda_{2}$. Then the following are equivalent:

(A) $\operatorname{Prrd}_{\kappa^{+}}^{1}\left(\lambda_{1}, \lambda_{2} ; \kappa, \theta\right)$.

(B) Assume $M$ is a model with a countable vocabulary, $\left|R_{l}^{M}\right|=\lambda_{l}$ for $l=$ $1,2, P^{M}=\kappa, Q^{M}=\theta, F^{M}$ is a 2-place function (really just $F\left\lceil\left(R_{1}^{M} \times R_{2}^{M}\right)\right.$ interests us), the range of $F \uparrow\left(R_{1}^{M} \times R_{2}^{M}\right)$ is included in $Q^{M}$ and $G$ is a function from $\left[R_{1}^{M}\right]^{*} \times\left[R_{2}^{M}\right]^{*}$ to $P^{M}$. Then we can find $\tau(M)$-models $M_{0}, N$ and elements $c^{*}, a_{\eta}, b_{\eta}\left(\right.$ for $\left.\eta \in{ }^{\omega} 2\right)$ such that: 
(i) $N$ is a model with the vocabulary of $M$ (but functions may be interpreted as partial ones, i.e. as relations),

(ii) $a_{\eta} \in R_{1}^{N}$ and $b_{\eta} \in R_{2}^{N}$ are pairwise distinct and $F^{N}\left(a_{\eta}, b_{\eta}\right)=$ $c^{*}(\in N)$,

(iii) $M_{0}$ is countable, $M_{0} \subseteq M, c^{*} \in Q^{M}, M_{0}$ is the closure of $\left(M_{0} \cap P^{M}\right) \cup\left\{c^{*}\right\}$ in $M$, in fact for some $M_{0}^{\prime} \prec M$ we have $M_{0}=$ closure of $P^{M_{0}^{\prime}} \cup\left\{c^{*}\right\}, c^{*} \in M_{0}^{\prime}$,

(iv) $M_{0} \subseteq N, P^{M_{0}}=P^{N}$,

(v) $|N|=\left\{\sigma\left(a_{\eta}, b_{\nu}, \bar{d}\right): \sigma\right.$ is a $\tau(M)$-term, $\eta \in{ }^{\omega} 2, \nu \in{ }^{\omega} 2$ and $\left.\bar{d} \subseteq M_{0}\right\}$

(vi) for $\left\{\eta_{l}: l<l(*)\right\},\left\{\nu_{m}: m<m(*)\right\} \subseteq{ }^{\omega} 2$ (both without repetitions nonempty) there is $d^{1} \in P^{M_{0}}$ such that if $\bar{d} \subseteq P^{M_{0}}$ and quantifier free formulas $\varphi_{l, m}$ are such that

$$
N \models \bigwedge_{l<l(*), m<m(*)} \varphi_{l, m}\left[a_{\eta_{l}}, b_{\nu_{m}}, \bar{d}\right]
$$

then for some $\left\{a_{l}: l<l(*)\right\} \subseteq R_{1}^{M}$ and $\left\{b_{m}: m<m(*)\right\} \subseteq$ $R_{2}^{M}$ (both with no repetition) we have

$$
M \models \bigwedge_{l<l(*), m<m(*)} \varphi_{l, m}\left[a_{l}, b_{m}, \bar{d}\right]
$$

and $G\left(\left\{a_{l}: l<l(*)\right\},\left\{b_{m}: m<m(*)\right\}\right)=d^{1}$,

(vii) for every quantifier free first order $\varphi=\varphi\left(x, y, z_{0}, \ldots\right) \in$ $L(\tau(M))$ and $d_{0}, \ldots \in M_{0}$ there is $k<\omega$ such that for every $\eta_{1}, \eta_{2}, \nu_{1}, \nu_{2} \in{ }^{\omega} 2$ such that $\eta_{1}\left\lceil k=\eta_{2}\left\lceil k\right.\right.$ and $\nu_{1}\left\lceil k=\nu_{2}\lceil k\right.$ we have

$$
N \models \varphi\left[a_{\eta_{1}}, b_{\nu_{1}}, d_{1}, \ldots\right]=\varphi\left[a_{\eta_{2}}, b_{\nu_{2}}, d_{1}, \ldots\right],
$$

$(\text { vii })^{+}$for every $n<\omega$, first order $\varphi=\varphi\left(x_{0}, \ldots, x_{n-1}\right) \in L(\tau(M))$ quantifier free and $d_{2}, d_{3}, \ldots \in M_{0}$ there is $n^{*}<\omega$ such that for every $k \in\left(n^{*}, \omega\right), \eta_{0}, \eta_{1} \in{ }^{\omega} 2, \nu_{0}, \nu_{1} \in{ }^{\omega} 2$ satisfying

$$
\eta_{0}\left\lceil k=\eta_{1}\left\lceilk \quad \text { and } \quad \nu _ { 0 } \left\lceil k=\nu_{1}\lceil k\right.\right.\right.
$$

we have

$$
N \models \varphi\left[a_{\eta_{0}}, b_{\nu_{0}}, d_{2}, \ldots\right] \equiv \varphi\left[a_{\eta_{1}}, b_{\nu_{1}}, d_{2}, \ldots,\right],
$$

(viii) if $\varphi$ is an existential sentence in $\tau(M)$ satisfied by $N$ then $\varphi$ is satisfied by $M$.

$(\mathrm{B})^{-}$Like (B) without $\left(\right.$vii) ${ }^{+}$, (viii).

Pro of. (B) $)^{-} \Rightarrow(\mathrm{A})$. Toward a contradiction assume $\operatorname{NPrrd}_{\kappa^{+}}\left(\lambda_{1}, \lambda_{2} ; \kappa\right)$. Hence there is a model $M^{\prime}$ witnessing it, so $\left|\tau\left(M^{\prime}\right)\right| \leq \kappa$. So $c \in Q^{M} \Rightarrow$ $\operatorname{rkrc}^{1}\left(M^{\prime}, c ; \kappa\right)<\kappa^{+}$(note that Prrd was defined by cases of $\operatorname{rkrc}(M, c, \kappa)$ ). 
Let $\left\{\varphi_{i}(x, y): i<\kappa\right\}$ list the quantifier free formulas in $L_{\omega, \omega}\left(\tau\left(M^{\prime}\right)\right)$ with free variables $x, y$. Let $\left\{u_{i}: i<\kappa\right\}$ list the finite subsets of $\kappa$. For $c \in$ $Q^{M^{\prime}}$ and $a_{0}, \ldots, a_{l(*)-1} \in R_{1}^{M^{\prime}}, b_{0}, \ldots, b_{m(*)-1} \in R_{2}^{M^{\prime}}\left(\bar{a}=\left\langle a_{0}, \ldots, a_{l(*)-1}\right\rangle\right.$, $\bar{b}=\left\langle b_{0}, \ldots, b_{m(*)-1}\right\rangle$ and we set $\left.a_{n+1+l}=b_{l}\right)$ let

$$
\alpha_{c, \bar{a}, \bar{b}}=\operatorname{rkrc}^{1}\left(\left(\left\{a_{0}, \ldots, a_{l(*)-1}\right\},\left\{b_{0}, \ldots, b_{m(*)-1}\right\}\right), M^{\prime}, c ; \kappa\right),
$$

and let $k_{c, \bar{a}, \bar{b}}, \varphi_{c, \bar{a}, \bar{b}}$ be witnesses for $\operatorname{rkrc}^{1}\left((\bar{a}, \bar{b}), M^{\prime}, c ; \kappa, \theta\right) \nsupseteq \alpha_{c, \bar{a}, \bar{b}}+1$. Let $i(c, \bar{a}, \bar{b})<\kappa$ be such that $\varphi_{c, \bar{a}, \bar{b}}$ is a conjunction of formulas of the form $\varphi_{j}\left(x_{l}, y_{m}\right)$ for $j \in u_{i(c, \bar{a}, \bar{b})}$. We define $M$ :

- the universe is $\left|M^{\prime}\right|$,

- the function $F^{M^{\prime}}$, relations $R_{1}^{M^{\prime}}, R_{2}^{M^{\prime}}, Q^{M^{\prime}}, P^{M^{\prime}}$, the pairing function on ordinals,

- $R_{n}=\left\{(i, a, b): a \in R_{1}^{M}, b \in R_{2}^{M}\right.$ and if $\left|u_{i}\right|>n$ then $M \models \varphi_{j}[a, b]$ where $j$ is the $n$th member of $\left.u_{i}\right\}$,

- let $H_{c}$ be one-to-one from $\omega \times \operatorname{rkrc}\left(M^{\prime}, c ; \kappa, \theta\right) \times \kappa$ into $\kappa$,

- we define the function $G$ :

$$
G_{c}(\bar{a}, \bar{b})=H\left(G_{c, 0}(\bar{a}, \bar{b}), G_{c, 1}(\bar{a}, \bar{b}), G_{c, 2}(\bar{a}, \bar{b})\right)=H\left(k_{c, \bar{a}, \bar{b}}, \alpha_{c, \bar{a}, \bar{b}}, i_{c, \bar{a}, \bar{b}}\right) .
$$

Now we can apply statement $(\mathrm{B})^{-}$of 4.6 which we are assuming and get $M_{0}, N, c^{*}, a_{\eta}, b_{\eta}$ (for $\eta \in{ }^{\omega} 2$ ) satisfying clauses (i)-(vii) there. So $c^{*} \in M_{0} \subseteq$ $M^{\prime} \cap N$, hence $\beta^{*}=\operatorname{rkrc}\left(M^{\prime}, c ; \kappa\right)$ satisfies $\beta^{*}<\infty$, even $<\kappa^{+}$. Clearly $\operatorname{rkrc}^{1}\left(N, c^{*} ; \kappa\right) \leq \beta^{*}$.

Consider all sequences

$$
\begin{array}{r}
\left\langle\left\langle\eta_{l}: l<l(*)\right\rangle,\left\langle\nu_{m}: m<m(*)\right\rangle, d_{1}, \bar{d},\left\langle\varphi_{l, m}: l<l(*), m<m(*)\right\rangle,\right. \\
\left.\left\langle a_{l}: l<l(*)\right\rangle,\left\langle b_{m}: m<m(*)\right\rangle\right\rangle
\end{array}
$$

which are as in clause (vi) of (B).

Among those tuples choose one with $\alpha^{*}=\operatorname{rkrc}^{1}\left(\left\{a_{l}: l<l(*)\right\},\left\{b_{m}\right.\right.$ : $\left.m<m(*)\}, N, c^{*} ; \kappa\right)$ minimal. Let this rank not being $\geq \alpha^{*}+1$ be exemplified by $\varphi$ and $k<l(*)+m(*)$, so by symmetry we can assume $k<l(*)$.

Choose $k^{*}<\omega$ large enough for clause (vi) of (B) to hold for all formulas $\varphi(x, y)$ appearing in $\left\{\varphi_{j}(x, y): j \in u_{i_{c^{*}, \bar{a}, \bar{b}}}\right\}$ where $i_{c^{*}, \bar{a}, \bar{b}}=G_{c^{*}, 2}(\bar{a}, \bar{b})$ and $\left\langle\eta_{l}\left\lceil k^{*}: l<l(*)\right\rangle,\left\langle\nu_{m}\left\lceil k^{*}: m<m(*)\right\rangle\right.\right.$ are with no repetition. Choose $\eta_{l(*)} \in 2^{\omega} \backslash\left\{\eta_{k}\right\}$ such that $\eta_{l(*)}\left\lceil k^{*}=\eta_{k}\left\lceil k^{*}\right.\right.$. Now apply clause (vi) of (B) ${ }^{-}$ to $\bar{\eta}^{\prime}=\left\langle\eta_{l}: l \leq l(*)\right\rangle, \bar{\nu}^{\prime}=\left\langle\nu_{m}: m<m(*)\right\rangle, \bigwedge_{m} \varphi_{l(*), m}\left(x_{l(*)}, x_{m}\right), d_{1}^{\prime}, \bar{d}^{\prime}$. By the choice of $\varphi$, these clearly satisfy $\operatorname{rkrc}^{1}\left(\left\{a_{l}^{\prime}: l<l(*)\right\},\left\{b_{m}^{\prime}: m<\right.\right.$ $\left.m(*)\}, N, c^{*} ; \kappa, \bar{\theta}\right)<\operatorname{rkrc}^{1}\left(\left\{a_{l}: l<l(*)\right\},\left\{b_{m}: m<m(*)\right\}, N, c^{*} ; \kappa, \bar{\theta}\right)=$ $\alpha^{*}$, but this easily contradicts the minimality of $\alpha^{*}$.

$(\mathrm{A}) \Rightarrow(\mathrm{B})$. As in the proof of $2.5,2.1$ (with a fixed $c$ ).

$(\mathrm{B}) \Rightarrow(\mathrm{B})^{-}$. Trivial. 
Discussion 4.7. (1) When applying $4.6(\mathrm{~A}) \Rightarrow(\mathrm{B})$, or 2.5 we can use $M$ which is an expansion of $\left(\mathcal{H}(\chi), \in,<^{*}\right)$ by Skolem functions, $P^{M}=\kappa, \chi$ large enough, so for $\eta, \nu \in{ }^{\omega} 2, N_{\eta, \nu}:=\operatorname{cl}_{N}\left(M_{0} \cup\left\{a_{\eta}, a_{\nu}\right\}\right)$ is a model of ZFC, not well founded but with standard $\omega$ and more: its $\{i: i<\kappa\}$ is a part of the true $\kappa$. In [Sh 532] we will have (as in 2.1) $M_{0} \subseteq N, M_{0} \prec M, M_{0} \prec N_{\eta, \nu}$, and if $N_{\eta, \nu}=" \varphi(\eta, \nu), \nu, \eta$ are (essentially) in ${ }^{\omega} 2, \varphi \in M_{0}$ is a $\kappa$-Suslin relation", then $\mathbf{V}=\varphi(\eta, \nu)$.

(2) We can give a rank to subsets of $\lambda_{1} \times \lambda_{2}$ and have parallel theorems.

Claim 4.8. If $\varphi \subseteq{ }^{\omega} 2 \times{ }^{\omega} 2$ is $\bigvee_{i<\theta} \varphi_{i}$, each $\varphi_{i}$ is $\kappa$-Suslin, $\varphi$ contains $a\left(\lambda_{1}, \lambda_{2}\right)$-rectangle, and $\operatorname{Prrd}_{\kappa^{+}+1}^{1}\left(\lambda_{1}, \lambda_{2} ; \theta\right)$ then $\varphi$ contains a perfect rectangle.

Pr o o f. Let $\varphi_{i}(\eta, \nu)=(\exists \varrho)\left((\eta, \nu, \varrho) \in \lim \left(T_{i}\right)\right)$ where $T_{i}$ is a $(2,2, \kappa)$-tree. Let $M$ be $\left(\mathcal{H}(\chi), \in,<_{\chi}^{*},\left\langle T_{i}: i<\theta\right\rangle, h, \Upsilon, \lambda_{1}, \lambda_{2}, R_{1}, R_{2}, Q, n\right)_{n<\omega}$ expanded by Skolem functions, where $Q^{M}=\theta$ and, for some $\eta_{i} \in \omega_{2} 2$ for $i<\lambda_{1}$ pairwise distinct and $\nu_{j} \in \omega_{2} 2$ for $j<\lambda_{2}$ pairwise distinct, $R_{1}^{M}=\left\{\eta_{i}\right.$ : $\left.i<\lambda_{1}\right\}$ and $R_{2}^{M}=\left\{\nu_{j}: j<\lambda_{2}\right\}$; finally, let $\Upsilon, h$ be functions such that $\left(\eta_{i}, \nu_{j}, \Upsilon\left(\eta_{i}, \nu_{j}\right)\right) \in \lim \left(T_{h\left(\eta_{i}, \nu_{j}\right)}\right)$. So let $N, M_{0}, c^{*}, a_{\eta}$ (for $\left.\eta \in{ }^{\omega} 2\right), b_{\eta}$ (for $\left.\eta \in{ }^{\omega} 2\right)$ be as in clause (B) of 4.6. Now $M$ has elimination of quantifiers, so there are quantifier free formulas $\varphi_{n}^{l}(x)$ saying (in $M$ ) that $x \in R_{l} \& x(n)$ $=1$, and let $H_{n}(x, y)$ be such that $x \in R_{1}^{M} \& y \in R_{2}^{M} \Rightarrow(\Upsilon(x, y))(n)=$ $H_{n}(x, y) \in \kappa=P^{M}$.

So for $\eta \in{ }^{\omega} 2$ we can define $\sigma_{\eta}^{1} \in{ }^{\omega} 2$ by $\sigma_{\eta}^{1}(n)=1 \Leftrightarrow N \models \varphi_{n}^{1}\left(a_{\eta}\right)$, and $\sigma_{\eta}^{1} \in \omega_{2}$ by $\sigma_{\eta}^{2}(n)=1 \Leftrightarrow N \models \varphi_{n}^{2}\left(b_{\eta}\right)$, and we define, for $\eta, \nu \in \omega_{2}$, a sequence $\sigma_{\eta, \nu} \in{ }^{\omega>}\left(P^{M_{0}}\right) \subseteq{ }^{\omega>} \kappa$ by $\sigma_{\eta, \nu(n)}=H_{n}\left(a_{\eta}, b_{\nu}\right)$.

Now $A:=\left\{\sigma_{\eta}^{1}: \eta \in{ }^{\omega} 2\right\}$ and $B:=\left\{\sigma_{\eta}^{2}: \eta \in{ }^{\omega} 2\right\}$ are perfect and for $\eta, \nu \in{ }^{\omega} 2,\left(\sigma_{\eta}^{1}, \sigma_{\eta}^{2}, \sigma_{\eta, \nu}\right) \in \lim (T)$, hence $A \times B$ is a perfect rectangle inside $\operatorname{prj} \lim (T)$.

FACT 4.9. (1) Assume that $\varphi \subseteq{ }^{\omega} 2 \times{ }^{\omega} 2$ is $\theta_{1}$-Suslin, $\kappa<\theta_{1}$ and $\theta=$ $\operatorname{cf}\left(\mathcal{S}_{\leq \kappa}\left(\theta_{1}\right), \subseteq\right)$. Then $\varphi$ can be represented as $\bigvee_{i<\theta} \varphi_{i}$ with each $\varphi_{i} \kappa$-Suslin.

(2) If $\varphi$ is co- $\kappa$-Suslin, then it can be represented as $\bigvee_{i<\kappa^{+}} \varphi_{i}$ with each $\varphi_{i} \kappa$-Borel (i.e. can be obtained from clopen sets by unions and intersections of size $\leq \kappa)$.

Proof. (1) Easy.

(2) Let $\neg \varphi$ be represented as $\operatorname{prj} \lim (T)$ with $T$ a $(2,2, \kappa)$-tree.

Now $\varphi(\eta, \nu)$ iff $T_{(\eta, \nu)}=\{\varrho:$ for some $n,(\eta\lceil n, \nu\lceil n, \varrho) \in T\}$ is well founded, which is equivalent to the existence of $\alpha<\kappa^{+}$and $f: T_{(\eta, \nu)} \rightarrow \alpha$ such that $\varrho_{1}<\varrho_{2} \in T \Rightarrow f\left(\varrho_{1}\right)>f\left(\varrho_{2}\right)$. For each $\alpha<\kappa^{+}$, this property is $\kappa$-Borel. 
ConClusion 4.10. If $\varphi$ is an $\aleph_{n}$-Suslin subset of ${ }^{\omega} 2 \times{ }^{\omega_{2}} 2$ containing a $\left(\lambda \operatorname{rd}_{\omega_{1}}\left(\aleph_{n}\right), \lambda \operatorname{rd}_{\omega_{1}}\left(\aleph_{n}\right)\right)$-rectangle then it contains a perfect rectangle. (Note: $\aleph_{n}$ can replaced by $\kappa$ if $\operatorname{cf}\left(\mathcal{S}_{\leq \aleph_{0}}(\kappa), \subseteq\right)=\kappa$, e.g. $\aleph_{\omega_{4}}$, by [Sh g, IX, §4].)

Conclusion 4.11. (1) For $l<6, \operatorname{Prrc}_{\infty}^{l}\left(\kappa^{+},\left(2^{\kappa^{+}}\right)^{+} ; \kappa\right)$.

(2) If $\mathbf{V}=\mathbf{V}_{0}^{\mathbf{P}}, \mathbf{P}=$ c.c.c. and $\mathbf{V}_{0}=G C H$ then $\operatorname{Prrc}_{\omega_{1}}\left(\aleph_{1}, \aleph_{3}\right)$.

Proof. (1) For a model $M$, letting $\left(\lambda_{1}, \lambda_{2}\right)=\left(\kappa^{+},\left(2^{\kappa^{+}}\right)^{+}\right)$choose (for $m=1,2)$ a nonempty sequence $\bar{a}_{i}^{m}$ from $R_{m}^{M}$ for $i<\lambda_{m}$, with $\left\{\bar{a}_{i}^{m}: i<\lambda_{m}\right\}$ pairwise disjoint. For $(i, j) \in \lambda_{1} \times \lambda_{2}$ let $\beta_{i, j}=\operatorname{rkrc}\left(\bar{a}_{i}^{1}, \bar{a}_{j}^{2}, M\right)$ with witnesses $k^{M}\left(\bar{a}_{i}^{1}, \bar{a}_{j}^{2}\right), \varphi^{M}\left(\bar{a}_{i}^{1}, \bar{a}_{j}^{2}\right)$ for $\neg \operatorname{rkrc}^{1}\left(\bar{a}_{i}^{1}, \bar{a}_{j}^{2}, M\right)>\beta_{i, j}$. As $\lambda_{2}=\left(2^{\lambda_{1}}\right)^{+}$and $|\tau(M)| \leq \kappa$, for some $B_{2} \subseteq \lambda_{2}$ with $\left|B_{2}\right|=\lambda_{2}$ and for every $i<\lambda_{1}$ the following does not depend on $j \in B_{2}$ :

$$
k^{M}\left(\bar{a}_{i}^{1}, \bar{a}_{j}^{2}\right), \varphi^{M}\left(\bar{a}_{i}^{1}, \bar{a}_{j}^{2}\right) .
$$

Similarly, there is $B_{1} \subseteq \lambda_{1}$ with $\left|B_{1}\right|=\lambda_{1}\left(=\kappa^{+}\right)$such that for $j=$ $\min \left(B_{2}\right)$ the values $k^{M}\left(\bar{a}_{i}^{1}, \bar{a}_{j}^{2}\right), \varphi^{M}\left(\bar{a}_{i}^{1}, \bar{a}_{j}^{2}\right)$ are the same for all $i \in B_{1}$; but they do not depend on $j \in B_{2}$ either. So for $(i, j) \in B_{1} \times B_{2}$ we have $k^{M}\left(\bar{a}_{i}^{1}, \bar{a}_{j}^{2}\right)=k^{*}, \varphi^{M}\left(\bar{a}_{i}^{1}, \bar{a}_{j}^{2}\right)=\varphi^{*}$. Let $k^{*}$ "speak" about $\bar{a}_{i}^{1}$, for definiteness only. Choose distinct $i_{\zeta}$ in $B_{1}$ (for $\zeta<\kappa^{+}$). We can assume $\operatorname{rkrc}^{1}\left(\bar{a}_{i_{0}}^{1}, \bar{a}_{j}^{1}, M\right) \leq \operatorname{rkrc}^{1}\left(\bar{a}_{i_{\zeta}}^{1}, \bar{a}_{j}^{1}, M\right)$.

Now $\bar{a}_{\zeta}^{1}$ give contradiction to $\operatorname{rkrc}^{l}\left(\bar{a}_{i_{0}}^{1}, \bar{a}_{j}^{2}, M\right) \ngtr \beta_{i_{0}, j}$.

(2) This can be proved directly (or see [Sh 532]) through preservation by c.c.c. forcing notion of ranks which are relatives of rkrc similarly to 1.10.

REMARK 4.12. (1) If $T$ is an $(\omega, \omega)$-tree and $A \times B \subseteq \lim (T)$ with $A, B \subseteq{ }^{\omega} \omega$ uncountable (or just not scattered) then $\lim (T)$ contains a perfect rectangle. Instead of $\lim (T)$ (i.e. a closed set) we can use a countable intersection of open sets. The proof is just like 1.17.

(2) We can define a rank for $(2,2, \kappa)$-trees measuring whether $\operatorname{prj} \lim (T)$ $\subseteq{ }^{\omega} 2 \times{ }^{\omega} 2$ contains a perfect rectangle, and similarly for $(\omega, \omega)$-trees $T$ measuring whether $\lim (T)$ contains a perfect rectangle. We then have theorems parallel to those of $\S 1$. See below and in [Sh 532].

The use of ${ }^{\omega} \omega$ below is just notational change.

Definition 4.13. For an $(\omega, \omega)$-tree $T$ we define a function $\operatorname{degrc}_{T}$ (rectangle degree). Its domain is $\operatorname{rcpr}(T):=\left\{\left(u_{1}, u_{2}\right)\right.$ : for some $l<\omega, u_{1}, u_{2}$ are finite nonempty subsets of ${ }^{l} \omega$ and $g$ a function from $u_{1} \times u_{2}$ to $\omega$ such that $\left(\eta_{0}, \eta_{1}\right) \in T$ for $\left.\eta_{i} \in u_{i}\right\}$. Its value is an ordinal $\operatorname{degrc}_{T}\left(u_{1}, u_{2}\right)$ (or -1 or $\infty)$. For this we define the truth value of $\operatorname{degrc}_{T}\left(u_{1}, u_{2}\right) \geq \alpha$ by induction on the ordinal $\alpha$.

CASE $1, \alpha=-1$ :

$$
\operatorname{degrc}_{T}\left(u_{1}, u_{2}\right) \geq-1 \quad \text { iff } \quad\left(u_{1}, u_{2}\right) \text { is in } \operatorname{rcpr}(T) .
$$


CASE 2, $\alpha$ limit:

$$
\operatorname{degrc}_{T}\left(u_{1}, u_{2}\right) \geq \alpha \quad \text { iff } \quad \operatorname{degrc}_{T}\left(u_{1}, u_{2}\right) \geq \beta \text { for every } \beta<\alpha .
$$

CASE $3, \alpha=\beta+1: \operatorname{degrc}_{T}\left(u_{1}, u_{2}\right) \geq \alpha$ iff for $k \in\{1,2\}$ and $\eta^{*} \in u_{k}$ we can find $l(*)<\omega$ and functions $h_{0}, h_{1}$ such that $\operatorname{Dom}\left(h_{i}\right)=u_{1} \cup u_{2},[\eta \in$ $\left.u_{1} \cup u_{2} \Rightarrow \eta \triangleleft h_{i}(\eta) \in{ }^{l(*)} \omega\right], h_{0}\left(\eta^{*}\right) \neq h_{1}\left(\eta^{*}\right), \eta \in u_{1-k} \Rightarrow h_{0}(\eta)=h_{1}(\eta)$ and letting $u_{i}^{1}=\operatorname{Rang}\left(h_{0}\left\lceil u_{i}\right) \cup \operatorname{Rang}\left(h_{1}\left\lceil u_{i}\right)\right.\right.$ we have $\operatorname{degrc}_{T}\left(u_{0}^{1}, u_{1}^{1}\right) \geq \beta$.

Lastly, define: $\operatorname{degrc}_{T}\left(u_{0}, u_{1}\right)=\alpha$ iff $\bigwedge_{\beta}\left[\operatorname{degrc}_{T}\left(u_{0}, u_{1}\right) \geq \beta \Leftrightarrow \alpha \geq \beta\right]$ $(\alpha$ an ordinal or $\infty)$.

Also $\operatorname{degrc}(T)=\operatorname{degrc}_{T}(\{\langle\rangle\},\{\langle\rangle\})$.

Claim 4.14. Assume $T$ is an $(\omega, \omega)$-tree.

(1) For every $\left(u_{0}, u_{1}\right) \in \operatorname{rcpr}(T)$, $\operatorname{degrc}_{T}\left(u_{0}, u_{1}\right)$ is an ordinal or $\infty$ or -1 ; if $f$ is an automorphism of $(\omega>\omega, \triangleleft)$ then

$$
\operatorname{degrc}_{T}\left(u_{0}, u_{1}\right)=\operatorname{degrc}_{f(T)}\left(f\left(u_{0}\right), f\left(u_{1}\right)\right) .
$$

(2) $\operatorname{degrc}(T)=\infty$ iff there is a perfect rectangle in $\lim (T)$ iff $\omega_{1} \leq$ $\operatorname{degrc}_{T}\left(u_{0}, u_{1}\right)$ for some $\left(u_{0}, u_{1}\right)$ (so those statements are absolute).

(3) If $\operatorname{degrc}(T)=\alpha(*)<\omega_{1}$ then $\lim (T)$ contains no $\left(\lambda \operatorname{rc}_{\alpha(*)+1}\left(\aleph_{0}\right)\right.$, $\left.\lambda \mathrm{rc}_{\alpha(*)+1}\left(\aleph_{0}\right)\right)$-rectangle.

(4) If $\bar{T}=\left\langle T_{n}: n\langle\omega\rangle\right.$ is a sequence of $(\omega, \omega)$-trees, $\operatorname{degrc}\left(T_{n}\right) \leq \alpha(*)$, and $A=\bigcup_{n<\omega} \lim \left(T_{n}\right)$ then $A$ contains no $\left(\lambda \operatorname{rc}_{\alpha(*)+1}\left(\aleph_{0}\right), \lambda \operatorname{rc}_{\alpha(*)+1}\left(\aleph_{0}\right)\right.$ rectangle.

(5) In part (4) we can replace $\omega$ by any infinite cardinal $\theta$.

Pro of. (1)-(3) Left to the reader.

(4) Follows from part (5).

(5) Let $\lambda=\lambda \operatorname{rc}_{\alpha(*)+1}(\theta), \bar{T}=\left\langle T_{i}: i<\theta\right\rangle$, $\operatorname{degrc}\left(T_{i}\right) \leq \alpha(*)$ and $A=\bigcup_{i<\theta} \lim \left(T_{i}\right)$. Let $\left\{\eta_{\alpha}: \alpha<\lambda\right\} \times\left\{\nu_{\beta}: \beta<\lambda\right\} \subseteq A$ where $\alpha<\beta \Rightarrow$ $\eta_{\alpha} \neq \eta_{\beta} \& \nu_{\alpha} \neq \nu_{\beta}$, and for simplicity $\left\{\eta_{\alpha}: \alpha<\lambda\right\} \cap\left\{\nu_{\beta}: \beta<\lambda\right\}=\emptyset$.

We define a model $M$, with universe $\mathcal{H}\left(\left(2^{\aleph_{0}}\right)^{+}\right)$and relations: all those definable in $\left(\mathcal{H}\left(\left(2^{\aleph_{1}}\right)^{+}\right), \in,<^{*}, R_{1}, R_{2}, g, \bar{T}, i\right)_{i \leq \theta}$ where $R_{1}^{M}=\left\{\eta_{\alpha}: \alpha<\lambda\right\}$, $R_{2}^{M}=\left\{\nu_{\beta}: \beta<\lambda\right\}$ and $g\left(\eta_{\alpha}, \nu_{\beta}\right)=\min \left\{i \leq \theta:\left(\eta_{\alpha}, \nu_{\beta}\right) \in \lim \left(T_{i}\right)\right\}$.

Next we prove

(*) if $w_{l} \in\left[R_{l}^{M}\right]^{*}$ for $l=1,2$, then

$$
\begin{aligned}
\operatorname{rkrc}\left(\left\langle w_{1}, w_{2}\right\rangle, M\right) \leq \min & \left\{\operatorname { d e g r c } _ { T _ { i } } \left(\left\{\eta_{\alpha}\left\lceil k: \alpha \in u_{1}\right\},\left\{\nu_{\beta}\left\lceil k: \beta \in u_{2}\right\}\right):\right.\right.\right. \\
& u_{1} \subseteq w_{1}, u_{2} \subseteq w_{2}, u_{1} \neq \emptyset, u_{2} \neq \emptyset, k<\omega, \\
& \left\langle\eta_{\alpha}\left\lceil k: \alpha \in u_{1}\right\rangle\right. \text { is with no repetitions, } \\
& \left\langle\nu_{\beta}\left\lceil k: \beta \in u_{2}\right\rangle \text { is with no repetitions }\right\} .
\end{aligned}
$$

We prove $(*)$ by induction on the left side of the inequality. Now by the definitions we are done. 4.14 
Claim 4.15. (1) For each $\alpha(*)<\omega_{1}$, there is an $\omega$-sequence $\bar{T}=\left\langle T_{n}\right.$ : $n<\omega\rangle$ of $(\omega, \omega)$-trees such that:

$(\alpha)$ for every $\mu<\lambda \operatorname{rc}_{\alpha(*)}\left(\aleph_{0}\right)$, some c.c.c. forcing notion adds a $(\mu, \mu)$-rectangle to $\bigcup_{n<\omega} \lim \left(T_{n}\right)$,

$(\beta) \operatorname{degrc}\left(T_{n}\right)=\alpha(*)$.

(2) If $\operatorname{NPrrc}_{\alpha(*)}\left(\lambda_{1}, \lambda_{2} ; \aleph_{0}\right)$ then for some $(\omega, \omega)$-tree $T$, some c.c.c. forcing notion adds a $\left(\lambda_{1}, \lambda_{2}\right)$-rectangle to $\lim (T)$ such that $\alpha(*)=\operatorname{degrc}(\bar{T})$ (consequently, if $\operatorname{Prrc}_{\alpha(*)}\left(\lambda_{1}^{\prime}, \lambda_{1}^{\prime} ; \aleph_{0}\right)$ then there is no $\left(\lambda_{1}^{\prime}, \lambda_{1}^{\prime}\right)$-rectangle in $\lim (T))$.

(3) Moreover, we can have for the tree $T$ of (4): if $\mu<\lambda \mathrm{rc}_{\alpha(*)}\left(\aleph_{0}\right)$ and $A, B$ are disjoint subsets of $\omega_{2} \times{ }^{\omega} 2$ of cardinality $\leq \mu$, then some c.c.c. forcing notion $\mathbf{P}$ adds an automorphism $f$ of $(\omega>\omega, \triangleleft)$ such that $A \subseteq$ $\lim ^{*}[f(T)]$ and $B \cap \lim ^{*}[f(T)]=\emptyset$ (the $\lim ^{*}$ means closure under finite changes).

Proof. We define the forcing for part (2) and postpone the others to [Sh 532].

(2) It is enough to do it for successor $\alpha(*)$, say $\beta(*)+1$. It is like 1.13; we will give the basic definition and the new points. Let $M$ be a model as in

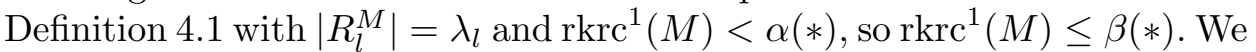
assume that $R_{1}^{M}$ are $R_{2}^{M}$ are disjoint sets of ordinals. For nonempty $\bar{a}_{l} \subseteq R_{l}^{M}$ $\left(l<2\right.$; no repetition inside $\left.\bar{a}_{l}\right)$, let $\varphi^{M}\left(\bar{a}_{1}, \bar{a}_{2}\right), k^{M}\left(\bar{a}_{1}, \bar{a}_{2}\right) \in \operatorname{Rang}\left(\bar{a}_{1}\right) \cup$ $\operatorname{Rang}\left(\bar{a}_{2}\right)$ be witnesses to the value of $\operatorname{rkrc}^{1}\left(\bar{a}_{1}, \bar{a}_{2}, M\right)$ which is $<\alpha(*)$.

We define the forcing notion $\mathbf{P}$ : a condition $p$ consists of:

(1) $\bar{u}^{p}=\left\langle u_{0}^{p}, u_{1}^{p}\right\rangle$ and $u_{\varepsilon}^{p}=u_{\varepsilon}[p]$ is a finite subset of $R_{\varepsilon}^{M}$ for $\varepsilon<2$.

(2) $n^{p}=n[p]<\omega$ and $\eta_{\alpha}^{p}=\eta_{\alpha}[p] \in{ }^{n[p]} \omega$ for $\alpha \in u_{\varepsilon}^{p}$ such that $\alpha \neq \beta \in u_{\varepsilon}^{p} \Rightarrow \eta_{\alpha}^{p} \neq \eta_{\beta}^{p}$.

(3) $0<m^{p}<\omega$ and $t_{m}^{p} \subseteq \bigcup\left\{{ }^{l} \omega \times{ }^{l} \omega: l \leq n^{p}\right\}$ is closed under initial segments and such that the $\triangleleft$-maximal elements have length $n^{p}$ and \langle\rangle$\in t^{p}$.

(4) The domain of $f^{p}$ is $\left\{\bar{u}=\left(u_{1}, u_{2}\right)\right.$ : for some $l=l\left(u_{1}, u_{2}\right) \leq n^{p}$ and $m=m\left(u_{1}, u_{2}\right)<m^{p}$ we have $u_{\varepsilon} \subseteq t_{\varepsilon}^{p} \cap \omega$ and if $\left.\alpha_{1} \in u_{1}, \alpha_{2} \in u_{2}, \eta_{\alpha_{1}}^{p}\right\rceil l \in u_{1}$ and $\eta_{\alpha_{2}}^{p}\left\lceil l \in u_{2}\right.$ then $\left.g^{p}\left(\alpha_{1}, \alpha_{2}\right)=m\right\}$ and $f^{p}(\bar{u})=\left(f_{0}^{p}(\bar{u}), f_{1}^{p}(\bar{u}), f_{2}^{p}(\bar{u})\right) \in$ $\alpha(*) \times\left(u_{1} \cup u_{2}\right) \times L_{\omega_{1}, \omega}(\tau(M))$.

(5) A function $g^{p}: u_{1}^{p} \times u_{2}^{p} \rightarrow\left\{0, \ldots, m^{p}-1\right\}, m^{p}<\omega$.

(6) $t_{m}^{p} \cap{ }^{\left(n^{p}\right)} \omega=\left\{\left(\eta_{\alpha}^{p}, \eta_{\beta}^{p}\right): \alpha \in u_{0}^{p}, \beta \in u_{1}^{p}\right.$ and $\left.m=g^{p}(\alpha, \beta)\right\}$.

(7) If $\emptyset \neq u_{\varepsilon} \subseteq t_{\varepsilon} \cap{ }^{l} \omega, f^{p}\left(u_{1}, u_{2}\right)=\left(\beta^{*}, \varrho^{*}, \varphi^{*}\right), l<l(*) \leq n^{p}$, for $i=$ 0,1 a function $e_{i, \varepsilon}$ has domain $u_{\varepsilon},\left[(\forall l)\left(\varrho \in u_{\varepsilon} \Rightarrow \varrho \triangleleft e_{i, \varepsilon}(\varrho) \in t_{\varepsilon}^{p} \cap l(*) \omega\right)\right]$, $\left[\varrho^{*} \notin u_{\varepsilon} \& \varrho \in u_{\varepsilon} \Rightarrow e_{0, \varepsilon}(\varrho)=e_{1, \varepsilon}(\varrho)\right],\left[\varrho^{*} \in u_{\varepsilon} \Rightarrow e_{0, \varepsilon}\left(\varrho^{*}\right) \neq e_{1, \varepsilon}\left(\varrho^{*}\right)\right]$ and $f^{p}\left(e_{0,1}\left(u_{1}\right) \cup e_{1,1}\left(u_{1}\right), e_{0,2}\left(u_{2}\right) \cup e_{1,2}\left(u_{2}\right)\right)=\left(\beta^{\prime}, \varrho^{\prime}, \varphi^{\prime}\right)$ (so well defined) then $\beta^{\prime}<\beta^{*}$. 
(8) If $l \leq n^{p}, u_{\varepsilon} \subseteq u_{\varepsilon}^{p}$ are nonempty for $\varepsilon=1,2$, the sequence $\left\langle\eta_{\alpha}^{p}\lceil l\right.$ : $\left.\alpha \in u_{\varepsilon}\right\rangle$ is with no repetition, and $u_{\varepsilon}^{\prime}=\left\{\eta_{\alpha}^{p}\left\lceil l: \alpha \in u_{\varepsilon}\right\}\right.$ and $f^{p}\left(u_{1}^{\prime}, u_{2}^{\prime}\right)$ is well defined, then $f_{2}^{p}\left(u_{1}^{\prime}, u_{2}^{\prime}\right)=\varphi^{M}\left(u_{1}, u_{2}\right), f_{1}^{p}\left(u_{1}^{\prime}, u_{2}^{\prime}\right)=\eta_{\alpha}^{p}\lceil l$ where $\alpha$ is $k^{M}\left(u_{1}, u_{2}\right)$ and $f_{0}^{p}\left(u_{1}^{\prime}, u_{2}^{\prime}, h\right)=\operatorname{rkrc}\left(u_{1}, u_{2}, M\right)$.

(9) If $\left(u_{1}^{\prime}, u_{2}^{\prime}\right) \in \operatorname{Dom}\left(f^{p}\right)$ then there are $l, u_{1}, u_{2}$ as above.

(10) if $\left(\eta_{1}, \eta_{2}\right) \in t_{m}^{p} \cap\left({ }^{n} 2 \times{ }^{n} 2\right)$ then for some $\alpha_{1} \in u_{1}^{p}, \alpha_{2} \in u_{2}^{p}$ we have $g^{p}\left(\alpha_{1}, \alpha_{2}\right)=m$ and $\eta_{1} \unlhd \eta_{\alpha_{1}}^{p}, \eta_{2} \unlhd \eta_{\alpha_{2}}^{p}$. $\mathbf{m}_{4.14}$

REMARK 4.16. We can generalize 4.13-4.15 to Suslin relations.

\section{References}

[CK] C. C. Chang and J. H. Keisler, Model Theory, Stud. Logic Found. Math. 73, North-Holland, Amsterdam, 1973.

[EHMR] P. Erdős, A. Hajnal, A. Máté and R. Rado, Combinatorial Set Theory: Partition Relations for Cardinals, Stud. Logic Found. Math. 106, NorthHolland, Amsterdam, 1984.

[GcSh 491] M. Gilchrist and S. Shelah, Identities on cardinals less than $\aleph_{\omega}$, J. Symbolic Logic 61 (1996), 780-787.

[HrSh 152] L. Harrington and S. Shelah, Counting equivalence classes for co- $\kappa$-Suslin equivalence relations, in: Logic Colloquium '80 (Prague, 1980), D. van Dalen et al. (eds.), Stud. Logic Found. Math. 108, North-Holland, Amsterdam, 1982, 147-152.

[Ke71] H. J. Keisler, Model Theory for Infinitary Logic. Logic with Countable Conjunctions and Finite Quantifiers, Stud. Logic Found. Math. 62, NorthHolland, Amsterdam, 1971.

[Mo] M. Morley, Omitting classes of elements, in: The Theory of Models, NorthHolland, 1965, 265-273.

[Sh a] S. Shelah, Classification Theory and the Number of Nonisomorphic Models, Stud. Logic Found. Math. 92, North-Holland, Amsterdam, xvi+544 pp., 1978.

[Sh c] -, Classification Theory and the Number of Nonisomorphic Models, Stud. Logic Found. Math. 92, North-Holland, Amsterdam, xxxiv+705 pp., 1990.

[Sh e] - Non-Structure Theory, Oxford Univ. Press, in preparation.

[Sh g] -, Cardinal Arithmetic, Oxford Logic Guides 29, Oxford Univ. Press, 1994.

[Sh 8] -, Two cardinal compactness, Israel J. Math. 9 (1971), 193-198; see also Notices Amer. Math. Soc. 18 (1971), 425.

[Sh 18] —, On models with power-like orderings, J. Symbolic Logic 37 (1972), 247267.

[Sh 37] —, A two-cardinal theorem, Proc. Amer. Math. Soc. 48 (1975), 207-213.

[Sh 49] -, A two-cardinal theorem and a combinatorial theorem, ibid. 62 (1976), $134-136$.

[Sh 202] -, On co- $\kappa-$ Suslin relations, Israel J. Math. 47 (1984), 139-153.

[Sh 262] - , The number of pairwise non-elementarily-embeddable models, J. Symbolic Logic 54 (1989), 1431-1455.

[Sh 288] -, Strong partition relations below the power set: consistency, was Sierpinski right? II, in: Sets, Graphs and Numbers (Budapest, 1991), Colloq. Math. Soc. János Bolyai 60, North-Holland, 1992, 637-638. 
[Sh 532] S. Shelah, More on co- $\kappa$-Suslin equivalence relations, in preparation.

Institute of Mathematics

The Hebrew University

Jerusalem 91904, Israel
Mathematics Department

Rutgers University

New Brunswick, New Jersey 08854

U.S.A.

E-mail: shelah@math.huji.ac.il

Received 28 February 1994;

in revised form 23 December 1996 and 11 March 1998 